AEI-08-NNNN

LPTENS-08/68

DESY 08-193

\title{
Finite Volume Spectrum of 2D Field Theories from Hirota Dynamics
}

\author{
Nikolay Gromov ${ }^{\alpha}$, Vladimir Kazakov ${ }^{\beta}$ and Pedro Vieira ${ }^{\gamma}$ \\ a DESY Theory, Notkestr. 85 22603 Hamburg Germany; \\ II. Institut für Theoretische Physik Universität Hamburg Luruper Chaussee 149 22761 Hamburg Germany; \\ St.Petersburg INP, Gatchina, 188 300, St.Petersburg, Russia \\ nikgromov AT gmail.com \\ § Laboratoire de Physique Théorique de l'Ecole Normale Supérieure et l'Université Paris-VI, 75231, \\ Paris CEDEX-05, France; \\ kazakov AT lpt.ens.fr \\ $\gamma$ Max-Planck-Institut für Gravitationphysik, Albert-Einstein-Institut, Am Mühlenberg 1, 14476 Potsdam, \\ Germany; \\ Departamento de Física e Centro de Física do Porto Faculdade de Ciências da Universidade do Porto Rua \\ do Campo Alegre, 687, 4169-007 Porto, Portugal \\ pedrogvieira AT gmail.com
}

\begin{abstract}
We propose, using the example of the $O(4)$ sigma model, a general method for solving integrable two dimensional relativistic sigma models in a finite size periodic box. Our starting point is the so-called Y-system, which is equivalent to the thermodynamic Bethe ansatz equations of Yang and Yang. It is derived from the Zamolodchikov scattering theory in the cross channel, for virtual particles along the non-compact direction of the space-time cylinder. The method is based on the integrable Hirota dynamics that follows from the Y-system. The outcome is a nonlinear integral equation for a single complex function, valid for an arbitrary quantum state and accompanied by the finite size analogue of Bethe equations. It is close in spirit to the Destri-deVega (DdV) equation. We present the numerical data for the energy of various states as a function of the size, and derive the general Lüscher-type formulas for the finite size corrections. We also re-derive by our method the DdV equation for the $S U(2)$ chiral Gross-Neveu model.
\end{abstract}




\section{Contents}

1 Introduction and Summary $\quad 3$

2 TBA and Y-system for $O(4)$ sigma model, or $S U(2)$ Principal Chiral Field 7

2.1 The Model . . . . . . . . . . . . . . . . . . . . . . . . . . . . . . . 7

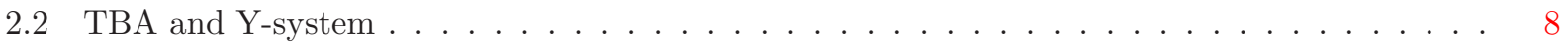

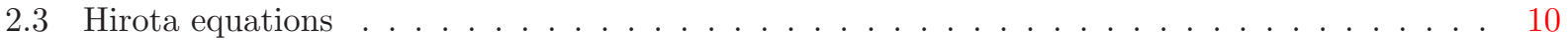

2.4 Asymptotic Bethe Ansatz and Classification of the Solutions _ . . . . . . . . . . . . . 12

2.5 Probing the finite volume $\ldots \ldots \ldots \ldots \ldots \ldots \ldots$

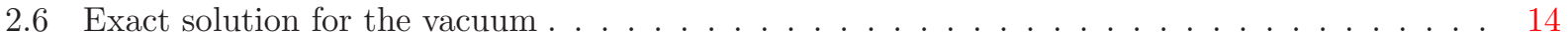

2.7 Generalization to $U(1)$ sector $\ldots \ldots \ldots \ldots \ldots \ldots \ldots \ldots$

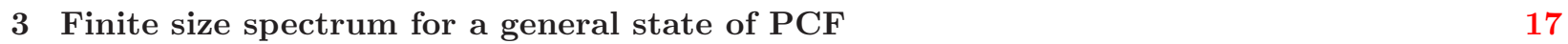

3.1 Exact equations for the finite volume spectrum $\ldots \ldots \ldots \ldots \ldots$

3.1 .1 Closed equation on the gauge function $g(x) \ldots \ldots \ldots \ldots \ldots \ldots \ldots$

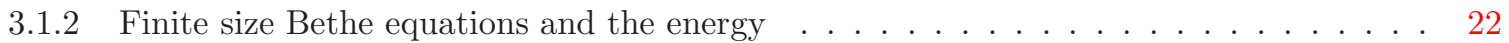

3.2 Large volume limit: ABA and Lüscher corrections _ . . . . . . . . . . . . . . . . 23

$3.2 .1 \quad$ Single particle case . . . . . . . . . . . . . . . . . . . . . 25

$4 S U(2)$ Chiral Gross-Neveu model and related models 26

5 Numerics $\quad 28$

5.1 Implementation of numerics and Mathematica code . . . . . . . . . . . . . . . 29

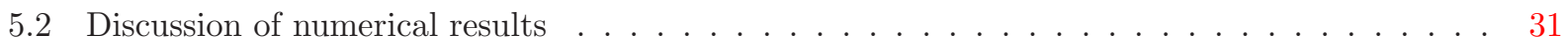

6 Conclusions $\quad 33$

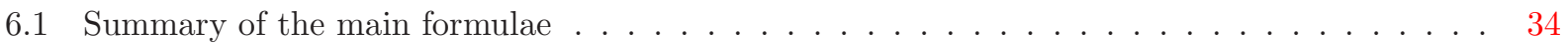

A Derivation of the (ground state) $Y$-system $\quad 35$

B General solution in terms of Hirota functions 41

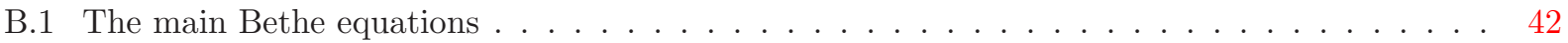

C Proof of reality of $T_{k} \quad 43$

D Proof of analyticity of $T_{-1}$ in the physical strip $\quad 44$

D.1 Analyticity in the $U(1)$ sector $\ldots \ldots \ldots \ldots \ldots \ldots$. . . . . . . . . . . 44

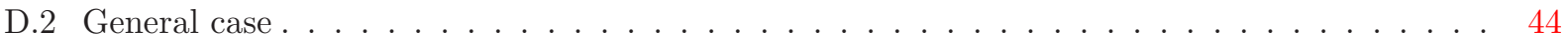

E Details on Lüscher formulae derivation $\quad 45$ 


\section{Introduction and Summary}

The study of the properties of Quantum Field Theories (QFT's) in finite volume, or at finite temperature, has a long history and numerous applications. Matsubara description [1] of finite temperature $T$ thermodynamics, by considering the system in the periodic imaginary time $t$, has lead to the extensive study of the Euclidean QFT's with one compactified dimension with numerous physical applications [2].

Lüscher found the leading finite size corrections to the mass gap in relativistic two dimensional QFT's [3, 4]. These corrections depend solely on the asymptotic S-matrix of the theory. Recently, Lüscher corrections to various multi-particle states in integrable 2D QFT were conjectured [5].

For the integrable 2D QFT's, as understood during the last two decades, the ambitions can be much higher: these systems are usually solvable at any finite size though a systematic approach to such solutions, as well as a good understanding of the working prescriptions, are still missing.

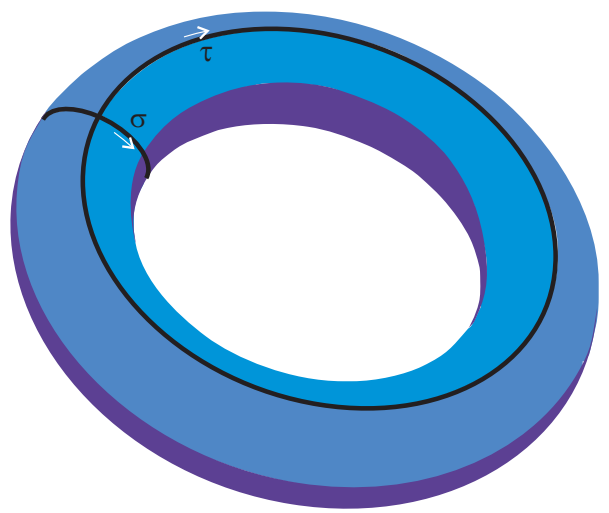

Figure 1: Physical channel, cross-channel and finite volume vs finite temperature.

There are two main schemes to address the finite size calculations. The first, pioneered by Destry and deVega (DdV) [6], is based on the integrable discretization. Once such discretization is at hand, the system can be studied by the well established methods based on the transfer matrix approach and the resulting non-linear integral equation (NLIE), often called the DdV equation, calculates not only the ground state energy but also the spectrum of excited states. The method appeared to be very powerful when applied to the Sine-Gordon model [7, 9, 11, 12, 13], or to more general RSOS models [14], Toda theories [15], hard hexagon models [16], etc.

However, for generic integrable QFT it is far from easy to find the corresponding integrable lattice regularization and for many models such discretization is not known. Nevertheless, the problem can be usually tackled by using a computation scheme alternative to the DdV approach. As explained in the seminal work of Al.Zamolodchikov [17] this is achieved by the double Wick rotation trick: using the Matsubara imaginary time formulation we can first find the free energy in the infinite volume but finite temperature. Next we flip the meaning of euclidian time and space directions on the cylinder: $\tau \rightarrow \sigma, \sigma \rightarrow \tau$, and interpret the free energy as the ground state of the system in finite volume $L=\frac{1}{T}$ (see fig.1). In this way we can obtain the exact finite volume ground state energy. This computational scheme is known by the name of Thermodynamic Bethe Ansatz (TBA).

The TBA equations, whose number is infinite in many interesting models, can be usually concisely casted into the so called Y-system functional equations $[18,19]$. Often the latter one can be 


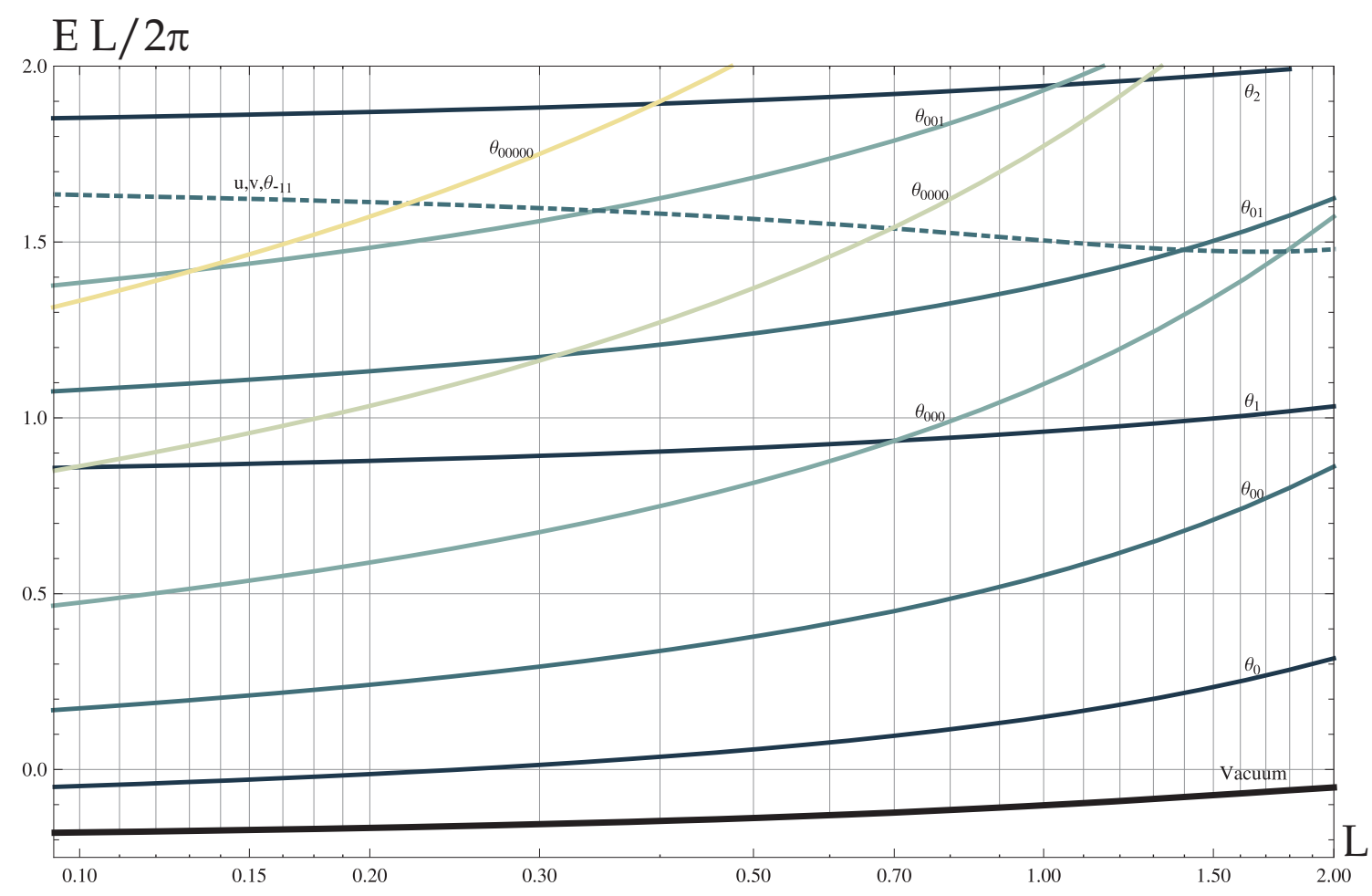

Figure 2: Plots of energies $E$ of a few excited states of $O(4)$ model on a circle of a circumference $L$. The vertical axis corresponds to the values of $\frac{L}{2 \pi} E$, the horizontal axis - to the values of $L$ in the logarithmic scale. The lowest curve depicts the vacuum energy. The next one, labeled as $\theta_{0}$, shows the mass gap energy. The corresponding state is in the $U(1)$ sector, with a single particle at rest, hence with the mode number $=0$. The next states in the $U(1)$ sector are denoted by $\theta_{n_{1} n_{2} n_{3}, \cdots}$, according to the mode numbers $n_{1}, n_{2}, n_{3}, \ldots$ excited for the 1-st, 2-nd, 3-rd, etc., particles. For all these states the $S U(2)_{L}$ and $S U(2)_{R}$ spins of the several particles are pointing in the same direction, say they are spin "up". The dashed line represents a state having a polarization out of the $U(1)$ sector, with left and right "magnons" excited - it corresponds to the quantum state of two particles where both $S U(2)_{L}$ and $S U(2)_{R}$ spins are in the singlet $s=0$ state. The qualitative explanation of these graphs will be given in subsection 5.2 .

rewritten in the form of DdV equations or some similar set of integral equations for a finite set of functions. The method was successfully used for many relativistic models [20, 21, 22, 23, 24]. As explained in the previous paragraph the computation of the exact ground state energy by means of this method is a relatively straightforward task with solid theoretical foundations.

To obtain the exact spectrum comprising all excited states of the theory is, on the other hand, a much more involved - and a very interesting - task. A possibility to describe the excited states within the TBA approach, by modifying the analytical properties of the thermodynamic functions, was first suggested in [24]. Another possible way to obtain the spectrum of the theory, proposed around the same time, is based on the analytic continuation of the ground state energy with respect to the parameters of the model, such as the mass or the chemical potential, in order to find the excited states [25]. If the integrable lattice regularization is absent, it is not well understood why these methods work. Nevertheless, the results are usually in the excellent agreement with the perturbation theory, Lüscher finite size corrections and the direct Monte-Carlo study for a wide range of sizes $L$ (see for example [26, 27, 28, 29, 30] for $O(n)$ and related $\sigma$-models). 


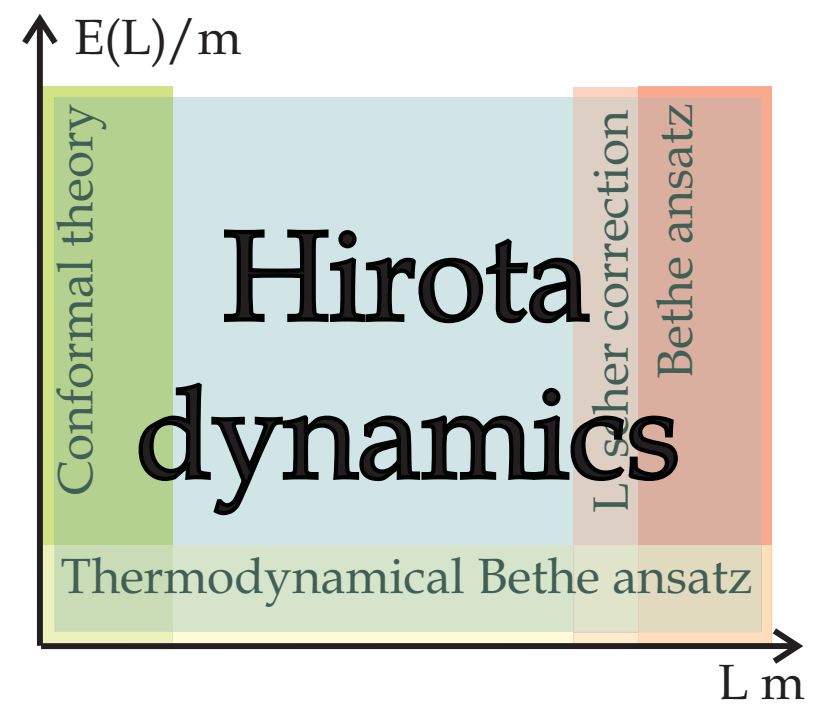

Figure 3: Domains of applicability of different descriptions of an integrable field theory at a finite volume $L$. In the ultra-violet regime, for small volume measured in units of a dynamically generated mass, the theory could be described by a conformal theory. In the infrared, at large volume, one can use the asymptotic Bethe equations. The leading order finite size corrections are governed by the (generalized) Lüscher corrections. At any volume but for the ground state energy only one can use Thermodynamical Bethe ansatz. Hirota equation, equivalent to Y-system but more efficient when it comes to imposing appropriate analyticity properties, is a universal tool covering the whole diagram.

For models with diagonal scattering, like the Sinh-Gordon theory [31], the whole classification of excited states is possible [32]. The situation is much more complex when we deal with the nondiagonal scattering. The nested structure of the corresponding Bethe ansatz equations leads to complicated magnon-type excitations and bound states. Little is known about the excited states in such finite size systems. The only models where the polarized excited states were investigated, using the DdV equations, are the Sine-Gordon model [7] and its supersymmetric version [11] as well as the tricritical Ising model [10]. By the existing methods only the sectors with diagonal scattering can be studied efficiently, as was done for example for the $O(4)$ sigma model in [30]. A general and unified description of all excited states of the $\sigma$-models like $O(n)$ or the $S U(N)$ principal chiral field (PCF), and similar ones, having a "geometric" target space, is still absent.

The main goal of the present paper is to give a method of a general and systematic description of all the states of integrable QFT's in finite volume. We will explain how to go beyond the asymptotic spectrum and compute the full finite size spectrum comprising all excited states of integrable sigma models. We do it here on the example of $O(4)$ sigma model and also for the $S U(2)$ chiral GrossNeveu model but our formalism is certainly more general and is most probably applicable to any integrable $1+1$ dimensional $\sigma$-models. The main ingredients of the method are:

- The two-particles S-matrix for integrable system allows us to write the periodicity condition quantizing the momenta of the physical particles on a large circle of length $R$. The equations following from the periodicity condition are so called asymptotic Bethe ansatz (ABA) equations describing all states of the model. The details of this computation for the $S O(4)$ sigma 
model are given in Appendix $\mathrm{A}^{1}$. They are, however, valid only in a sufficiently big volume compared to the typical interaction distance, $R m \gg 1$ where $m$ is the infinite volume mass gap.

- For the ground state, the double Wick rotation $(\sigma, \tau) \rightarrow(\tau, \sigma)$ allows to reduce the problem to the thermodynamics. One can put the euclidian theory on the torus with one radius, $R$, very large and another one, $L$, arbitrary (see the fig.1). The ground state energy for a finite radius is related to the thermodynamic partition function. The exact equations for it can be found using the asymptotic spectrum given in the cross channel by the asymptotic Bethe equations. The resulting infinite series of integral equations, thermodynamic Bethe ansatz (TBA) equations, are casted into a functional form called Y-system. Here is the main assumption: we assume that different solutions of the $\mathrm{Y}$-system describe not only the ground state but all the excited states. One should furthermore restrict the class of solutions by assuming certain analytic properties which will in particular identify the quantum numbers of the states we are considering.

- Classical integrability of the Y-system, as a finite difference equation equivalent to the Hirota difference equation [34, 19], allows us to express explicitly the infinite number of the unknown functions through a finite number of the basic ones [35, 36].

- The Baker-Akhiezer function of the Lax pair associated with the Hirota equation can be interpreted as the Baxter function encoding the "magnon" Bethe roots, responsible for the $S U(2)_{R}$ and $S U(2)_{L}$ polarizations of states. The analyticity properties important for the full formulation of the resulting non-linear integral equation, are also suggested by the Lax equations. The gauge symmetry of Hirota equations allows to explicitly fix the final nonlinear integral equation (NLIE) for each state of the theory.

The resulting equation can be studied in various limits (such as Lüscher finite size corrections or small volume, conformal limit) or solved numerically in a rather efficient way. The fig.2 shows some of our numerical results obtained from the new equation, plotting the energy of various states as functions of the volume. When the similar results are available in the literature the agrement is perfect.

The general scheme elaborated in this paper on the example of the $O(4)$ sigma-model should be applicable to all integrable relativistically symmetric 2D QFT's. It should be also useful for the study of finite size effects when the system does not look explicitly relativistic but allows the S-matrix description and this S-matrix obeys the crossing symmetry, like the AdS/CFT S-matrix $[37,38]$. Y-system and Hirota equations give a unified and powerful point of view at all this subject since they solve in an almost trivial way the "kinematic" part of the problem related to the representation theory, whatever is the symmetry or supersymmetry of the model [35, 39].

Our method based on Hirota equation, being exact for any finite size $L$ of the system, reproduces well various limiting cases (see the fig.3). For the large $L$, the energies of the states are well described by the Lüscher corrections $[3,4,5]^{2}$. We derive them here for a general state with arbitrary polarization, which is also a new result, extending some hypothesis existing in the literature [5]. For small $L$, our results are well described by the theory of three free bosons, as will be discussed

\footnotetext{
${ }^{1}$ We are unaware of the existence of such derivation of the Y-system for the PCF in the literature

${ }^{2}$ Actually, as we will see from our numerics, Lüscher corrections work surprisingly well all the way until $L m \sim 1$.
} 
in the paper. The results for various low-lying levels, including the cases of non-diagonal scattering which are new, are summarized in the fig.2.

Our resulting NLIE can be brought sometimes to a form similar to the DdV equation. In the cases when the latter is available it can even coincide with DdV equation (an example of the chiral Gross-Neveu model is considered in our paper). It would be extremely interesting to understand the relation between the solution based on the integrable lattice discretization of [40] and our proposed integral equations. Nevertheless we should stress that the real power of our method should be in its universality: it should work in all situations when the TBA equations in the form of the Y-system are available.

\section{TBA and Y-system for $O(4)$ sigma model, or $S U(2)$ Principal Chiral Field}

The method we are proposing it quite general and we hope that a wide range of models could be solved using it. However for the sake of simplicity we will exemplify it on the $S U(2)$ Principal Chiral Field (PCF), equivalent to the $O(4)$ sigma model. In section 4 we will also consider the $S U(2)$ Chiral Gross-Neveu model.

\subsection{The Model}

The action of the PCF is given by the usual expression

$$
\mathcal{S}_{\sigma}=\frac{1}{e_{0}^{2}} \int d t d x\left(\partial_{\alpha} X_{a}\right)^{2}, \quad \sum_{a=1}^{4}\left(X_{a}\right)^{2}=1,
$$

whose target space is $S^{3}$. It is equivalent to the $S U(2) \otimes S U(2)$ principal chiral field (PCF) whose infinite volume solution was given in [41, 42, 43]. Indeed, by packing the fields $X_{i}$ into an $S U(2)$ group element $h=X_{4}+i \sum_{j=1}^{3} X_{j} \sigma_{j}$ with $\sigma_{j}$ being the usual Pauli matrices, we can re-write the action $\operatorname{as}^{3}$

$$
\mathcal{S}_{\mathrm{PCF}}=-\frac{1}{2 e_{0}^{2}} \int d t d x \operatorname{tr}\left(h^{-1} \partial_{\alpha} h\right)^{2} .
$$

The spectrum of this asymptotically free theory in the infinite volume consists of a single physical particle of mass $m=\Lambda e^{-\frac{2 \pi}{e_{0}^{2}}}$, where $\Lambda$ is a cut-off. Its wave function transforms in the fundamental representation under each of the $S U(2)$ subgroups. Al. and A.Zamolodchikov [33] proposed the exact elastic scattering matrix for such particles:

$$
\hat{S}_{12}(\theta)=S_{0}(\theta) \frac{\hat{R}(\theta)}{\theta-i} \otimes S_{0}(\theta) \frac{\hat{R}(\theta)}{\theta-i}, \quad S_{0}(\theta)=i \frac{\Gamma\left(\frac{1}{2}-\frac{i \theta}{2}\right) \Gamma\left(+\frac{i \theta}{2}\right)}{\Gamma\left(\frac{1}{2}+\frac{i \theta}{2}\right) \Gamma\left(-\frac{i \theta}{2}\right)},
$$

where $\hat{R}(\theta)$ is the usual $S U(2)$ R-matrix in the fundamental representation given by $\hat{R}(\theta)=\theta+i \hat{P}$ and $\hat{P}$ is the permutation operator exchanging the spins of the particles being scattered. This

\footnotetext{
${ }^{3}$ In the $A d S / C F T$ literature one usually uses $\sqrt{\lambda}=\frac{4 \pi}{e_{0}^{2}}$.
} 
S-matrix was established due to: (i) analyticity, (ii) unitarity, (iii) absence of bound states, (iv) crossing. In particular, (ii) and (iv) lead to the following identity

$$
S_{0}(\theta+i / 2) S_{0}(\theta-i / 2)=\frac{\theta-i / 2}{\theta+i / 2}
$$

on the scalar (dressing) factor. We can use this S-matrix to study the spectrum of $N$ particles in a periodic space circle of a sufficiently big circumference $L \gg m^{-1}$. The spectrum can be defined from the wave function periodicity condition

$$
\prod_{j=k+1}^{N} \hat{\mathcal{S}}\left(\theta_{k}-\theta_{j}\right) \prod_{j=1}^{k-1} \hat{\mathcal{S}}\left(\theta_{k}-\theta_{j}\right)|\Psi\rangle=e^{-i m L \sinh \left(\pi \theta_{k}\right)}|\Psi\rangle,
$$

which quantizes the momenta of the physical particles. The asymptotic spectrum of the theory put on a large circle of length $L$ is then given by

$$
E=\sum_{j=1}^{N} m \cosh \left(\pi \theta_{j}\right)
$$

where $\theta_{j}$ are solutions to the Bethe equation (see Appendix A for more details). In what follows we will measure all dimensional quantities in the units of $m$. Diagonalizing the periodicity condition (5) in the physical space by the usual methods (see an appendix in [39] for this model) we get the main Bethe equation

$$
e^{-i L \sinh \left(\pi \theta_{j}\right)}=-\prod_{k} S_{0}^{2}\left(\theta_{j}-\theta_{k}\right) \frac{\mathcal{Q}_{u}\left(\theta_{j}+i / 2\right)}{\mathcal{Q}_{u}\left(\theta_{j}-i / 2\right)} \frac{\mathcal{Q}_{v}\left(\theta_{j}+i / 2\right)}{\mathcal{Q}_{v}\left(\theta_{j}-i / 2\right)} .
$$

The magnon rapidities $u_{j}$ and $v_{j}$ are fixed by the auxiliary Bethe equations

$$
-\frac{\mathcal{Q}_{u}\left(u_{j}+i\right)}{\mathcal{Q}_{u}\left(u_{j}-i\right)}=\frac{\phi\left(u_{j}+i / 2\right)}{\phi\left(u_{j}-i / 2\right)},-\frac{\mathcal{Q}_{v}\left(v_{j}+i\right)}{\mathcal{Q}_{v}\left(v_{j}-i\right)}=\frac{\phi\left(v_{j}+i / 2\right)}{\phi\left(v_{j}-i / 2\right)},
$$

where

$$
\mathcal{Q}_{w}(x)=\prod_{j}\left(x-w_{j}\right), \quad \text { for } \quad w=u, v
$$

and $\phi(x)=\prod_{j}\left(x-\theta_{j}\right)$.

\subsection{TBA and Y-system}

As we mentioned in the introduction, the ground state energy $E_{0}(L)$ for arbitrary $L$ can be computed starting from asymptotical Bethe ansatz in the cross-channel. For $S U(2)$ principal chiral field this is described in detail in the Appendix A. The output is that the ground state energy is given by

$$
E_{0}(L)=-\frac{1}{2} \int d \theta \cosh (\pi \theta) \log \left(1+Y_{0}\right),
$$

where $Y_{0}$ is one out of an infinite number of $Y$-functions $Y_{n}$ with $n \in \mathbb{Z}$ obeying the TBA-type equations

$$
\log Y_{n}+L \cosh (\pi x) \delta_{n 0}=s * \log \left(1+Y_{n+1}\right)\left(1+Y_{n-1}\right), \quad n=0 \pm 1, \pm 2, \ldots
$$


with $s=\frac{1}{2 \cosh (\pi x)}$ and the sign $*$ denoting the convolution. If $\log \left(Y_{n}(x)\right)$ for any $n$ have no singularities inside the physical strip $-1 / 2<\operatorname{Im} x<1 / 2$ we can easily invert the operator $s *$ to get simply $s^{-1}=e^{\frac{i}{2} \partial_{x}}+e^{-\frac{i}{2} \partial_{x}}$ and these integral equations can be rewritten in a functional, $Y$-system form

$$
Y_{n}^{+} Y_{n}^{-}=\left(1+Y_{n+1}\right)\left(1+Y_{n-1}\right),
$$

supplemented with the asymptotic boundary conditions for large $x$

$$
Y_{n} \sim e^{-L \cosh (\pi x) \delta_{n 0}} \times \text { const }_{n} .
$$

The superscripts \pm stand for shifts of the argument by $\pm i / 2^{4}$,

$$
f^{ \pm} \equiv f(x \pm i / 2)
$$

Eq.(12) has however many solutions and only one of them really leads to the ground state energy.

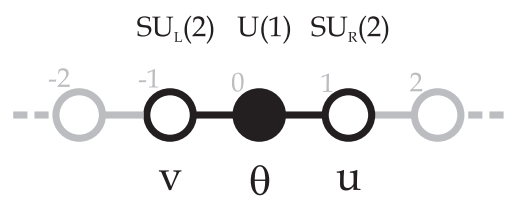

Figure 4: Dynkin diagram (three central nodes) and its extension for the magnon bound states (grey nodes) reflecting the structure of the Y-system. The central, black node corresponds to the $U(1)$ sector excitations of the model ( $\theta$-roots), the upper and lower nodes correspond to the more general states for magnon excitations for the $S U(2)_{L}$ wing (u-roots) and the $S U(2)_{R}$ wing (v-roots).

It is commonly believed that certain other solutions there describe the excited states [25, 44]. The energy of the $N$-particle excited states is again given in terms of $Y_{0}$ but is modified

$$
E(L)=-\frac{1}{2} \int d \theta \cosh (\pi \theta) \log \left(1+Y_{0}\right)+\sum_{j=1}^{N} m \cosh \left(\pi \theta_{j}\right),
$$

where the extra terms are inspired by the analytic continuation in $L$ and the points $\theta_{j}$ [25] are singularities of the integrand in the first term

$$
Y_{0}\left(\theta_{j} \pm i / 2\right)=-1, \quad j=1,2, \ldots, N .
$$

As we shell see, the last equation is nothing but the Bethe ansatz equation for physical rapidities modified at the finite volume. The last term in (15) is generated from the integral (10) by picking up the logarithmic poles (16).

Our goal in this section is to make use of the integrability of the Y-system rewriting it in the form of classical integrable discrete Hirota dynamics. This allows us to write down explicitly a solution for all $Y_{n}$ in terms of a finite number of functions. Then we will restrict ourself to a certain sub-class of physically relevant solutions with particular analytic properties. The analyticity will allow us to fix the functions completely and parameterize all the physical solutions for the excited $N$ particle states in terms of a finite set of complex parameters, Bethe roots, restricted by supplementary Bethe equations reducing in the infinite volume to the usual Bethe equation.

\footnotetext{
${ }^{4}$ We will often use even a more general notation, like $\overbrace{f^{++\ldots+}}^{k}=f(x+i k / 2)$ or $\overbrace{f^{-\ldots-\ldots}}^{k}=f(x-$ $i k / 2)$.
} 


\subsection{Hirota equations}

The $Y$-system equations eq.(12) can be seen as a gauge invariant version of the so called Hirota equation or $T$-system

$$
T_{k}(x+i / 2) T_{k}(x-i / 2)-T_{k-1}(x) T_{k+1}(x)=\Phi\left(x+i \frac{k}{2}\right) \bar{\Phi}\left(x-i \frac{k}{2}\right) .
$$

It can be easily checked [19] that Hirota equation is equivalent to the $Y$-system eq.(12) if we denote

$$
Y_{k}(x)=\frac{T_{k+1}(x) T_{k-1}(x)}{\Phi\left(x+i \frac{k}{2}\right) \bar{\Phi}\left(x-i \frac{k}{2}\right)} .
$$

At first sight, this is just another trivial rewriting of the TBA equations, however the Hirota form appears to be particulary useful. Using Hirota equation we can also write

$$
1+Y_{k}(x)=\frac{T_{k}(x+i / 2) T_{k}(x-i / 2)}{\Phi\left(x+i \frac{k}{2}\right) \bar{\Phi}\left(x-i \frac{k}{2}\right)} .
$$

Let us point out here an important fact. By evaluating the above equation for $k=0$ at $\theta_{j} \pm i / 2$ where $\theta_{j}$ is a zero of $T_{0}$ we observe that

$$
T_{0}\left(\theta_{j}\right)=0 \Rightarrow Y_{0}\left(\theta_{j} \pm i / 2\right)=-1
$$

which is the Bethe ansatz eq.(16). We will use this fact to associate zeroes of $T_{0}$ with physical rapidities.

Since $Y_{k}(x)$ are real functions by their physical meaning (for ground state they are ratios of densities of complexes and of their holes, see Appendix A) we can restrict ourself to the case when $T_{k}$ are real functions and $\Phi$ and $\bar{\Phi}$ are complex conjugated functions. [35]

Hirota equation (17) is integrable and has a Lax representation through the auxiliary problem

$$
\begin{aligned}
T_{k+1}(x) Q\left(x+i \frac{k}{2}\right)-T_{k}\left(x-\frac{i}{2}\right) Q\left(x+i \frac{k}{2}+i\right) & =+\Phi\left(x+i \frac{k}{2}\right) \bar{Q}\left(x-i \frac{k}{2}-i\right) \\
T_{k-1}(x) \bar{Q}\left(x-i \frac{k}{2}-i\right)-T_{k}\left(x-\frac{i}{2}\right) \bar{Q}\left(x-i \frac{k}{2}\right) & =-\bar{\Phi}\left(x-i \frac{k}{2}\right) Q\left(x+i \frac{k}{2}\right) .
\end{aligned}
$$

The compatibility of these two equations for the bi-vector of functions $\{Q(x), \bar{Q}(x)\}$ leads to the initial Hirota equation. Here $\bar{Q}$ is the complex conjugate function to $Q$. Note that if $T_{k}(x)$ are real functions then the second equation is simply the complex conjugate of the first one after shifting $k \rightarrow k+1$ and $x \rightarrow x+i / 2$. Two particularly useful relations from this Lax representation are

$$
\begin{aligned}
T_{1}(x) & =T_{0}(x-i / 2) \frac{Q(x+i)}{Q(x)}+\Phi(x) \frac{\bar{Q}(x-i)}{Q(x)}, \\
T_{-1}(x) & =T_{0}(x+i / 2) \frac{Q(x)}{Q(x+i)}-\Phi(x) \frac{\bar{Q}(x)}{Q(x+i)},
\end{aligned}
$$

Note that the first relation in (22) is a generalization of the famous Baxter equation usually written for the spin chains. We will see that in the infinite volume limit $\Phi(x)=T_{0}(x+i / 2)$ and that 
these equations reduce to the usual Baxter equation for spin chains, where $T_{1}$ plays the role of the transfer matrix in fundamental representation for the magnons of the $S U_{R}(2)$ wing of the theory, whereas the second equation plays a similar role for the $S U_{L}(2)$ wing (see Fig.4).

The main advantage of the Lax equations (21) is that they are linear in $T_{k}$ and we can easily express any $T_{k}$ in terms of $T_{0}, \Phi$ and $Q$ in the explicit form [35]

$$
\begin{aligned}
T_{k}(x) & =\frac{Q\left(x+i \frac{k+1}{2}\right)}{Q\left(x-i \frac{k-1}{2}\right)} T_{0}(x-i k / 2) \\
& +Q\left(x+i \frac{k+1}{2}\right) \bar{Q}\left(x-i \frac{k+1}{2}\right) \sum_{j=1}^{k} \frac{\Phi\left(x-i \frac{k+1}{2}+i j\right)}{Q\left(x-i \frac{k-1}{2}+i j\right) Q\left(x-i \frac{k+1}{2}+i j\right)} .
\end{aligned}
$$

This leads to a quite general and explicit solution of the $Y$-system via eq.(18). A nice feature of this form is that one can efficiently analyze the $L \rightarrow \infty$ limit and reproduce the asymptotic spectrum described by BAE eqs. $(7,8)$. This will be the goal of the next section.

Hirota and Lax equations exhibit several important symmetries. First of all a discrete symmetry exchanging the $u$-wing and the $v$-wing (right and left $S U(2)$ ): $Y_{k} \leftrightarrow Y_{-k}$ is induced by

$$
T_{k} \leftrightarrow T_{-k}, \Phi \leftrightarrow-\bar{\Phi}, Q \leftrightarrow \bar{Q}^{--}, \bar{Q} \leftrightarrow Q^{++},
$$

which will be quite useful for our further constructions. Moreover, both equations (17) and (21) are invariant under the gauge transformation

$$
\begin{aligned}
T_{k}(x) & \rightarrow g\left(x+i \frac{k}{2}\right) \bar{g}\left(x-i \frac{k}{2}\right) T_{k}(x), \\
\Phi(x) & \rightarrow g(x-i / 2) g(x+i / 2) \Phi(x) \\
\bar{\Phi}(x) & \rightarrow \bar{g}(x-i / 2) \bar{g}(x+i / 2) \bar{\Phi}(x), \\
Q(x) & \rightarrow g(x-i / 2) Q(x) .
\end{aligned}
$$

To preserve the reality of $T_{k}$ we should assume that $\bar{g}$ is the complex conjugated function to $g$. These transformations leave $Y_{k}(x)$ invariant.

The general solution of Hirota equation (17) can be also presented in a determinant form [35]

$$
T_{k}(x)=h(x+i k / 2)\left|\begin{array}{ll}
Q\left(x+i \frac{k+1}{2}\right) & R\left(x+i \frac{k+1}{2}\right) \\
\bar{Q}\left(x-i \frac{k+1}{2}\right) & \bar{R}\left(x-i \frac{k+1}{2}\right)
\end{array}\right|
$$

where $h(x)$ is a periodic function: $h^{++} \equiv h(x+i)=h(x)$ and $Q, R$ are two linearly independent solutions of the Lax equations (21) related by the Wronskian relation

$$
\Phi(x)=h(x+i / 2)\left|\begin{array}{cc}
R(x) & Q(x) \\
R(x+i) & Q(x+i)
\end{array}\right| .
$$

This determinant form will be very useful when we will formulate the general solution of the finite size PCF system for any state. It is not absolutely necessary to use it, but it simplifies some derivations. 


\subsection{Asymptotic Bethe Ansatz and Classification of the Solutions}

The main problem in computing the exact spectrum of the $S U(2) \mathrm{PCF}$ is to find the physical solutions to the $Y$-system (12) or, alternatively, to the Hirota equation (17), i.e., obeying the right asymptotic properties (13). Their classification is a complicated task, especially when we want to take into account not only the excitations of $U(1)$ sector but also the "magnon" type excitations of $S U(2)_{L}$ and $S U(2)_{R}$ sectors. The goal of this section is thus to identify the large $L$ solutions to the $Y$-system (12). The discussion in this section is not completely rigorous since our only goal is to get an idea of how asymptotic Bethe ansatz (ABA) eqs. $(7,8)$ appears from the $Y$-system. Together with the expression (6) the ABA equations must appear from the large $L$ asymptotic of exact solutions, as yielding the leading order value of the full spectrum.

The main simplification in the large $L$ limit is that $Y_{0} \rightarrow 0$. From eq.(13) we see that $Y_{0} \rightarrow$ $2 e^{-L \cosh (\pi x)}$ and we are left with two decoupled chains of equations for $k>0$ and $k<0$ [13]. For each wing we can introduce two sets of $T_{k}$ describing the corresponding solutions of the whole $T$-system: $T_{k}^{u}$ and $T_{k}^{v}$ such that $Y_{k>0}\left(Y_{k<0}\right)$ can be expressed in terms of $T_{k}^{u}\left(T_{k}^{v}\right)$ by the formula (18). Then $Y_{0}=0$ implies

$$
T_{-1}^{u}=0, T_{1}^{v}=0 .
$$

Let us focus on $T_{k}^{u}$ since we can always use the wing exchange symmetry (24) to describe $T_{k}^{v}$.

We have to identify the solutions which will lead to the asymptotic spectrum described by the ABA. It turns out that in terms of Hirota potentials $T_{k}$ it is enough in this limit to make very simple assumptions, namely:

- All $T_{k>0}^{u}(x)$ are polynomials at $L \rightarrow \infty$. We denote in this limit $T_{0}^{u}(x) \approx \prod_{j}\left(x-\theta_{j}\right) \equiv \phi(x)$.

- $Q^{u}(x)$ is a polynomial with real roots which we denote $Q^{u}(x)=\prod_{j}\left(x-u_{j}\right)$.

Then from eq.(22) we see that

$$
\Phi^{u}(x)=T_{0}^{u}(x+i / 2) \text { and } T_{1}^{u}(x)=\frac{T_{0}^{u}(x+i / 2) Q^{u}(x-i)+T_{0}^{u}(x-i / 2) Q^{u}(x+i)}{Q^{u}(x)} .
$$

From the polynomiality condition for $T_{k}^{u}(x)$ and $T_{k}^{v}(x)$ we get precisely the auxiliary Bethe equations eq.(8).

Finally, we should note that eq.(7) for the physical rapidities $\theta_{j}$ is also satisfied. This follows from imposing $Y_{0}\left(\theta_{j} \pm i / 2\right)=-1$ for all zeros $\theta_{j}$ of $T_{0}^{u}$, see (20). At first sight, this seems to be impossible to satisfy since, as we noticed, $Y_{0}(x)$ is small. However this smallness appears because $Y_{0}$ is proportional to $e^{-L \cosh (\pi x)}$ which is indeed small inside the physical strip $-1 / 2<\operatorname{Im} x<1 / 2$ but is of order 1 on the boundary of this strip. To impose this condition we must first compute $Y_{0}$ to the next order.

From (12) at $n=0$ we get

$$
Y_{0}^{+} Y_{0}^{-}=\frac{T_{1}^{u+} T_{-1}^{v+} T_{1}^{u-} T_{-1}^{v-}}{\left(\phi^{++} \phi^{--}\right)^{2}}
$$

Defining $S(x)=\prod_{j=1}^{N} S_{0}\left(x-\theta_{j}\right)$ we have, from the crossing relation (4), $S^{++} S=\phi / \phi^{++}$, so that

$$
Y_{0}^{+} Y_{0}^{-}=\left(\frac{T_{1}^{u}(x) T_{-1}^{v}(x) S^{2}(x+i / 2)}{\phi^{2}(x-i / 2)}\right)^{+}\left(\frac{T_{1}^{u}(x) T_{-1}^{v}(x) S^{2}(x+i / 2)}{\phi^{2}(x-i / 2)}\right)^{-},
$$


from which we can identify $Y_{0}$ up to a zero mode factor of $y_{0}=e^{-L \cosh \pi x}$ which obeys $y_{0}^{+} y_{0}^{-}=1$. Such factor should be included into $Y_{0}$ to ensure the proper asymptotic (13). Thus we find

$$
Y_{0}(x) \simeq e^{-L \cosh (\pi x)} T_{1}^{u}(x) T_{-1}^{v}(x) \frac{S^{2}(x+i / 2)}{\phi^{2}(x-i / 2)} .
$$

Evaluating it at $x=\theta_{k}-i / 2$ and using eq.(29) we get

$$
-1 \simeq e^{i L \sinh \left(\pi \theta_{k}\right)} \frac{\mathcal{Q}_{u}\left(\theta_{k}+i / 2\right) \mathcal{Q}_{v}\left(\theta_{k}+i / 2\right)}{\mathcal{Q}_{u}\left(\theta_{k}-i / 2\right) \mathcal{Q}_{v}\left(\theta_{k}-i / 2\right)} \prod_{j} S_{0}^{2}\left(\theta_{k}-\theta_{j}\right)
$$

which is nothing but the main ABA equation (7) for the middle node in fig.4. We use here the notations

$$
\mathcal{Q}_{v}(x)=\bar{Q}_{v}(x-i), \mathcal{Q}_{u}(x)=Q_{u}(x),
$$

to make the $u$ - and $v$-wings more symmetric. The advantage of these notations is that the wing exchange symmetry eq.(24) simply exchanges $\mathcal{Q}_{v}$ and $\mathcal{Q}_{u}$ and in the large $L$ limit they are real polynomials.

Finally, since $Y_{0}(x)$ is exponentially suppressed for real $x$ we can drop the integral contribution in (15) which leaves us with the energy as a sum of energies of individual particles, precisely as expected from (6).

Notice that the Zamolodchikov asymptotic scattering theory is implicitly contained in the $Y$ system, as we see from the appearance of the scalar scattering factor $S^{2}$ in the formula (32).

\subsection{Probing the finite volume}

Now, having established the solution at infinite volume, we need an insight into the analytic properties of $T$-functions in a finite, though large, volume. Let us find perturbatively the finite $L$ corrections for the simplest vacuum solution which for large $L$ corresponds to $Q^{u}=Q^{v}=1, \phi=1$. From eq.(23) one can see that for this case, to the leading order, $T_{k}^{u} \simeq k+1$ which implies for $Y_{k} \simeq|k|^{2}+2|k|$. Thus we are looking for a solution in the form

$$
Y_{k}=|k|^{2}+2|k|+y_{k}, \quad k=-\infty, \ldots, \infty
$$

where the first two terms in the r.h.s. are the trivial solution at $L=\infty$, where as $y_{k} \sim Y_{0}$ are small. We will see that the solution for the perturbation is unique under the assumption that when $k \rightarrow \infty$ the perturbation goes to zero $y_{k} \rightarrow 0$. The linearized $Y$-system in the Fourier form is

$$
\frac{k}{k+2} \tilde{s} \tilde{y}_{k+1}-\tilde{y}_{k}+\frac{k+2}{k} \tilde{s} \tilde{y}_{k-1}=0, k \geq 0
$$

where $\tilde{y}_{k}$ is the Fourier transform of $y_{k}$ and $\tilde{s}=\frac{1}{2 \cosh (\omega / 2)}$ is the Fourier transform of the kernel $s=\frac{1}{2 \cosh (\pi \theta)} \cdot \tilde{y}_{0}=\tilde{Y}_{0}$ is a fixed function. We see that this is a second order recurrence equation which in general has two linear independent solutions. Fortunately it can be solved explicitly. ${ }^{5}$ The general solution reads

$$
\tilde{y}_{k}=\frac{k(k+1)(k+2)}{2}\left(\left[\frac{e^{-\frac{k|\omega|}{2}}}{k}-\frac{e^{-\frac{(k+2)|\omega|}{2}}}{k+2}\right] C_{1}(\omega)+\left[\frac{e^{\frac{k|\omega|}{2}}}{k}-\frac{e^{\frac{(k+2)|\omega|}{2}}}{k+2}\right] C_{2}(\omega)\right) .
$$

\footnotetext{
${ }^{5}$ One can use RSolve function in Mathematica to find the solution.
} 
The needed solution satisfying $\tilde{y}_{0}=\tilde{Y}_{0}, \tilde{y}_{\infty}=0$ corresponds to $C_{1}=\tilde{Y}_{0}, C_{2}=0$. Making the inverse fourier transformation we get

$$
y_{k}=\frac{k(k+1)(k+2)}{\pi}\left(\frac{1}{4 x^{2}+k^{2}}-\frac{1}{4 x^{2}+(k+2)^{2}}\right) * Y_{0} .
$$

It can be easily checked that the approximate $T_{k}$ yielding this solution through (18) are

$$
T_{k-1}^{u}=T_{1-k}^{v} \simeq k+\frac{k / \pi}{4 x^{2}+k^{2}} * Y_{0}, \quad k \geq 0 .
$$

and

$$
\Phi(x)=1+\frac{1 / \pi}{4(x+i / 2+i 0)^{2}+1} * Y_{0} .
$$

The $i 0$ in this expression can be dropped when computing $Y_{k>0}$ from (18) but is included in this expression so that (18) can also be used for $k=0$, for more details see the discussion in the next subsection.

An important feature of this asymptotic solutions for $T_{k}$, which should persist at any $L$, is that it acquires two branch cuts at $x \in \mathbf{R} \pm i k / 2$ when $L \rightarrow \infty{ }^{6}$

\subsection{Exact solution for the vacuum}

We will now extend the solution found in the previous section to arbitrary $L$. First, we notice that the solution in terms of $T_{k}$ is much simpler than in terms of $Y_{k}$. For the vacuum we can use the following ansatz inspired by eq.(38)

$$
T_{k-1}=k+\frac{k / \pi}{4 x^{2}+k^{2}} * f, \quad k=+0,1,2, \ldots
$$

where $f$ is some function which for large $L$ becomes $Y_{0}$. One can easily see from the linear system eq.(21) at $Q=\bar{Q}=1$ that this ansatz solves the Hirota equation and can be presented in the form eq.(23) with $\Phi(x)=T_{0}(x+i / 2+i 0)$. Thus the Y-system equations eq.(12) for $|k| \geq 2$ are satisfied automatically. Notice that none of the $T_{k}$ 's has singularities on the real axis, which is of course a necessary feature of the solution: the physical quantities $Y_{k}$ should not be singular there.

To check that the equation for $k=1$ is also satisfied we have to define $Y_{0}$ in terms of $T_{k}$. For that we can simply analytically continue eq.(40) to the point $k=+0$ which gives $T_{-1}(x)=f(x) / 2$. We also have $\Phi(x)=T_{0}(x+i / 2+i 0), \bar{\Phi}(x)=T_{0}(x-i / 2-i 0)$ as mentioned above. These properties are supported by the second equation (22) which can be viewed as yielding the spectral density in terms of a jump on any of two infinite cuts. Then we get

$$
Y_{0}(x)=\frac{T_{0}(x+i / 2-i 0) T_{0}(x-i / 2+i 0)}{T_{0}(x+i / 2+i 0) T_{0}(x-i / 2-i 0)}-1=\frac{T_{1}(x) f(x) / 2}{T_{0}(x+i / 2+i 0) T_{0}(x-i / 2-i 0)}
$$

This equation relates $Y_{0}$ and $f$. With $Y_{0}$ so defined the Y-system equations at $|k|=1$ are now also satisfied. However the equation (11) for $k=0$ is still not used. Using

$$
\left(1+Y_{1}\right)\left(1+Y_{-1}\right)=\left(1+Y_{1}\right)^{2}=\left(\frac{T_{1}(x+i / 2) T_{1}(x-i / 2)}{T_{0}(x+i) T_{0}(x-i)}\right)^{2}
$$

\footnotetext{
${ }^{6}$ The term "branch cut" is not very appropriate here since the infinite cut has no branch points. However, as we shall see, a spectral representation will allow us to define $T_{k}(x)$ in the whole complex plane in terms of spectral density integrals along the cuts.
} 
and recalling that $s$ is the inverse shift operator we obtain ${ }^{7}$

$$
Y_{0}(x)=e^{-L \cosh (\pi x)} \frac{T_{1}^{2}(x)}{\left[T_{0}(x+i) T_{0}(x-i)\right]^{* 2 s}} .
$$

Combining it with eq.(41) we get

$$
f(x)=2 T_{1}(x) \frac{T_{0}(x+i / 2+i 0) T_{0}(x-i / 2-i 0)}{\left[T_{0}(x+i) T_{0}(x-i)\right]^{* 2 s}} e^{-L \cosh (\pi x)},
$$

which, in virtue of the eq.(40), gives a closed equation for $f(x)$.

Notice that from eq.(43) $T_{-1}(x)=f(x) / 2$ is exponentially small for large $L$ with

$$
T_{-1}(x) \simeq 2 e^{-L \cosh (\pi x)} .
$$

The finite $L$ solution to equation (43) can be easily found by iterations, starting from this large $L$ asymptotic and gradually diminishing $L$. We solved this equation numerically and get a perfect match with the existing results (see the Tab.1 comparing our results with [26]).

\begin{tabular}{llll}
$L$ & Leading order & Eq.(43) & Results of [26] \\
\hline$L=4$ & -0.015513 & -0.015625736 & $-0.01562574(1)$ \\
$L=2$ & -0.153121 & -0.162028968 & $-0.16202897(1)$ \\
$L=1$ & -0.555502 & -0.64377457 & $-0.6437746(1)$ \\
$L=1 / 2$ & -1.364756 & -1.74046938 & $-1.7404694(2)$ \\
$L=1 / 10$ & -7.494391 & -11.2733646 & $-11.273364(1)$
\end{tabular}

Table 1: We solve numerically eq.(43) the use $Y_{0}$ from eq.(41) to compute the energy of the ground state using eq.(10).

In the next subsection, we generalize this solution to the excited states in the $U(1)$ sector.

\subsection{Generalization to $U(1)$ sector}

In this section we will study in detail the $U(1)$ sector of the theory where we consider the states with $N$ particles with the same polarization, i.e. with no magnon excitations. Hence we can put all $Q=1$. As mentioned before - see eq. (20) - for $N$ particle states we expect $T_{0}\left(\theta_{j}\right)=0$ for each of $N$ rapidities of the particles $\theta_{1}, \ldots, \theta_{N}$.

In the previous section the vacuum state, with no particles excited, was analyzed. We saw that $T_{0}(x)$ inside the physical strip, $\Phi(x)$ above the strip and $\bar{\Phi}(x)$ below the strip could be described by a single function

$$
F(x)=1+\frac{1 / \pi}{4 x^{2}+1} * T_{-1},
$$

such that

$$
F(x)=\left\{\begin{array}{ll}
\Phi(x-i / 2) & , \operatorname{Im}(x)>1 / 2 \\
T_{0}(x) & ,|\operatorname{Im}(x)|<1 / 2 \\
\bar{\Phi}(x+i / 2) & , \operatorname{Im}(x)<-1 / 2
\end{array} .\right.
$$




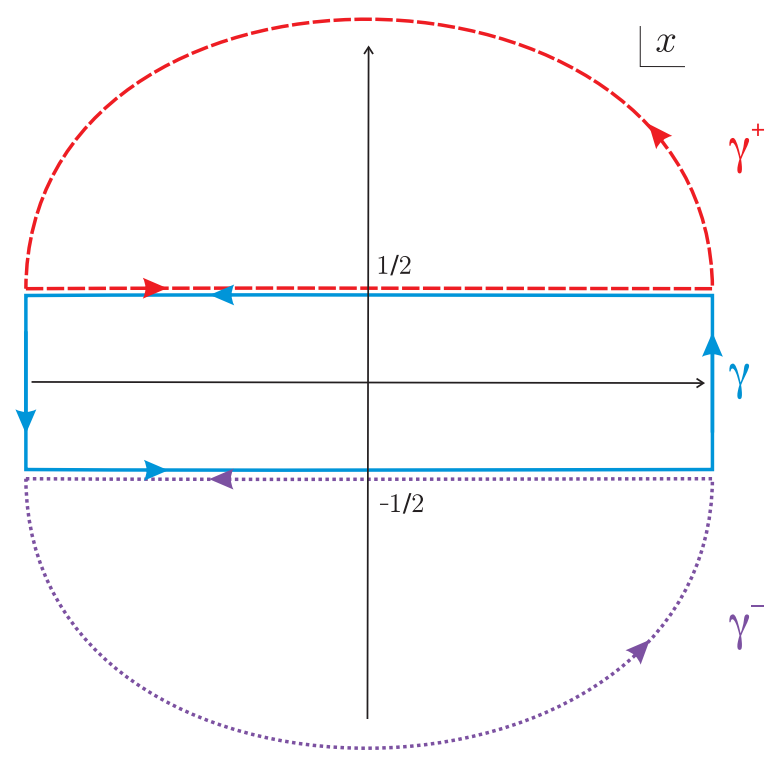

Figure 5: The function $F(x)$ in (47) can be recast as a contour integral as in (49) with the contours as represented in this figure.

Here we build a generalization of (45) for the case when $T_{0}$ has an arbitrary number of zeroes inside the physical strip for which (46) holds:

$$
F(x)=\phi(x)\left(1-\int_{-\infty}^{\infty}\left(\frac{1}{\phi(y-i / 2)} \frac{1}{x-y+i / 2}-\frac{1}{\phi(y+i / 2)} \frac{1}{x-y-i / 2}\right) \frac{T_{-1}(y) d y}{2 \pi i}\right),
$$

with $\phi(x) \equiv \prod_{j=1}^{N}\left(x-\theta_{j}\right)$. The overall factor of $\phi(x)$ appears because $T_{0}\left(\theta_{j}\right)=0$. The spectral representation of $F(x)$ as two integrals over the two infinite cuts at $\operatorname{Im}(x)= \pm 1 / 2$ is inspired by (45) and can be also seen from the linear problem (21). Indeed, we have

$$
T_{-1}(x)=T_{0}(x+i / 2)-\Phi(x)=T_{0}(x-i / 2)-\bar{\Phi}(x)
$$

which justifies the choice of spectral densities used in (47). To see that (46) indeed holds we write (47) as

$$
F(x)=\phi(x)\left(\oint_{\gamma} \frac{d y}{2 \pi i} \frac{T_{0}(y) / \phi(y)}{y-x}+\oint_{\gamma^{+}} \frac{d y}{2 \pi i} \frac{\Phi(y-i / 2) / \phi(y)}{y-x}+\oint_{\gamma^{-}} \frac{d y}{2 \pi i} \frac{\bar{\Phi}(y+i / 2) / \phi(y)}{y-x}\right) .
$$

The contours $\gamma, \gamma^{+}$and $\gamma^{-}$encircle respectively the physical strip, the region above the strip and the region below the strip, see figure 5 . For this relation to be equivalent to (47) we require that for large $x$ we should have $T_{0}(x), \Phi(x-i / 2), \bar{\Phi}(x+i / 2) \rightarrow \phi(x)$ at $|x| \rightarrow \infty$ along the corresponding contour. Finally, for (46) to hold, the ratios in (49) should be analytic inside the corresponding contours. Notice that at large $L$ the function $T_{-1}$ is exponentially small and thus $\Phi^{-}, T_{0}, \bar{\Phi}^{+} \rightarrow \phi(x)$ as expected from our discussion in section 2.4, to get the ABA equations. The large $x$ limit should be similar to the large $L$ limit since the source term in the $Y$-system $e^{-L \cosh (\pi x)}$ is small in both cases.

\footnotetext{
${ }^{7}$ We introduce a natural notation $g^{* s} \equiv e^{s * \log g}$.
} 
Let us now consider the other Hirota functions $T_{k}$. From (21) we have $T_{1}(x)=T_{0}(x+i / 2)+$ $\bar{\Phi}(x)=\Phi(x)+T_{0}(x-i / 2)$ which in terms of the function $F(x)$ reads

$$
T_{1}(x)=F(x+i / 2+i 0)+F(x-i / 2+i 0)=F(x+i / 2-i 0)+F(x-i / 2-i 0),
$$

so it is indeed regular on the real axis. Notice that $T_{1}$ is regular at least inside the enlarged strip $|\operatorname{Im}(x)|<1$. In the same way we can easily see that $T_{k>0}$ is analytic inside the strip $|\operatorname{Im}(x)|<\frac{k+1}{2}$.

Having expressed $T_{0}, \Phi$ and $T_{1}$ in terms of $T_{-1}$ through the function $F(x)$ we can find a closed equation of $T_{-1}$ from the $Y$-system equation for $n=0$. The derivation is parallel to the one in the previous section and it leads to

$$
T_{-1}(x)=(F(x+i / 2)+F(x-i / 2)) \frac{F(x+i / 2+i 0) F(x-i / 2-i 0)}{[F(x+i) F(x-i)]^{2 s}} e^{-L \cosh (\pi x)},
$$

supplemented by the quantization condition $Y_{0}\left(\theta_{j}+i / 2\right)=-1$. As before, the solution to these equations can be easily found from iterations as is explained in the Sec.5. The numerically calculated energies of a few states of this $U(1)$ sector are presented on the fig.2.

In the next section, we generalize these results to any excited states including the magnon polarizations. We will use a different strategy and incorporate the gauge invariance of $Y$-system to find the solutions of Y-system eq.(12) matching the $L=\infty$ asymptotic of the Sec.2.4.

\section{Finite size spectrum for a general state of PCF}

We will now describe how to construct the solution for the most general state of the PCF at finite volume $L$, having an arbitrary number of physical particles with arbitrary polarizations in the $S U(2)_{R}$ and $S U(2)_{L}$ wings (characterized by left and right "magnons" $u_{i}$ and $v_{i}$ ). Our method is based on the following observations and steps:

- We know from eq.(32) the structure of the poles and zeroes of all $Y_{k}$ 's in the limit $L \rightarrow \infty$ when $Y_{0}=0$. We assume that this structure will qualitatively persist even for finite $L$, and the classification of the appropriate solutions of the Y-system will follow the same pattern of poles and zeroes.

- We will recast the Y-system in terms of T-system (Hirota equation) since the analytic structure of $T_{k}$ 's is much simpler than of $Y_{s}$ as we saw from the vacuum solution (38) at $L \rightarrow \infty$.

- For any "good" solution of $Y$-system there is a family of solutions of Hirota equations related by gauge transformations (25). Hirota equation can be solved explicitly in terms of $T_{0}$, $\Phi$ and $Q$ as in eq.(23).

- For $L \rightarrow \infty$ we have two independent solutions for $T_{k}$ 's as we saw in the previous section. For one solution $T_{k}^{u}$ are asymptotically polynomials for $k>0$ and for another one $T_{k}^{v}$ with $k<0$ are polynomials when $L$ is large. We can then smoothly continue these two solutions to finite $L$ 's using the gauge freedom to preserve polynomiality of $Q$ 's.

- We have two global solutions of Hirota equation which can be parameterized by $T_{0}^{u}, \Phi_{u}, Q^{u}$ , and by $T_{0}^{v}, \Phi_{v}, Q^{v}$. They represent however the same and unique solution of the Y-system and thus should be related by a gauge transformation $g: T_{s}^{v}=g \circ T_{s}^{u}$, see (25). 
- Using certain assumptions about analyticity of $T_{0}^{u}$ and $\Phi_{u}$, supported by the Lax equations (21), we can express them as different analytic branches of the same analytic function $G_{u}$. The same can be done for $T_{0}^{v}$ and $-\bar{\Phi}_{v}$ in terms of $G_{v}$.

- The solution will be completely fixed by the existence of such a gauge transformation $g(x)$ which relates its $u$ - and the $v$-representations. At the end we will have one single non-linear integral equation (NLIE) on $g(x)$.

The final equation for $g(x)$ is new for the Principal Chiral Field. It is different from the system of $3 \mathrm{DdV}$ type equations used for the same model in [30]. Still it resembles in many aspects the non-linear Destri-de Vega (DdV) equation which appears when studying other integrable models. Indeed, our method is very general and it allows to generate DdV-like equations for large classes of sigma models in a systematic way. For the models for which a DdV equation is known we expect our integral equation to coincide with it after an appropriate change of variables. We check this hypothesis on the $S U(2)$ chiral Gross-Neveu model for which we re-derive indeed the known integral equation.

\subsection{Exact equations for the finite volume spectrum}

In this section, we will derive the finite volume spectral equations of the previous section in the most general form, valid for all excited states of the model with any number of physical particles with arbitrary polarizations (i.e. with any quantum numbers).

As we discussed below in the infinite volume, the solution of Y-system with $Y_{0}=0$ can be described in terms of two independent sets of Hirota potentials $T_{k}^{u}$ and $T_{k}^{v}$. Since these two different solutions of Hirota equation correspond to the same solution of Y-system they are related by a gauge transformation $g(x)$. These two solutions of Hirota equation can be continuously and unambiguously deformed all the way from very large $L$, where we know the solution (see the previous section), to any finite value of $L$. The gauge ambiguity for any of the two solutions, $T_{k}^{u}$ or $T_{k}^{v}$, can be fixed by choosing $Q_{u}$ and $Q_{v}$ to be polynomials for any $L$. Of course we can no longer assume $T_{k}^{u}$ and $T_{k}^{v}$, as well as the corresponding $\Phi_{v}$ and $\Phi_{u}$, to be polynomials. Instead we will assume certain analytic properties for them and we will see their consistency with the solution we find at the end.

We introduce a polynomial $\phi(x)$ with real zeroes $\theta_{j}, j=1,2, \ldots, N$ of $T_{0}^{u}$. They correspond to the rapidities of physical particles on the circle. The gauge function $g(x)$ relating the two solutions of the $T$-system is assumed to be regular and to have no zeros on the physical strip, so that $T_{0}^{v}=g \bar{g} T_{0}^{u}$ has the same zeroes as $T_{0}^{u}$ there. We also assume that ${ }^{8}$

- $\frac{\Phi_{u}(x)}{\phi(x+i / 2)}\left(\frac{\bar{\Phi}_{u}(x)}{\phi(x-i / 2)}\right)$ is regular for $\operatorname{Im} x>-1 / 2(\operatorname{Im} x<1 / 2)$ in the whole upper (lower) half plane and goes to 1 for $|x| \rightarrow \infty$ in all directions in the upper (lower) half plane;

- $\frac{T_{0}^{u}(x)}{\phi(x)}$ is regular and goes to 1 at $x \rightarrow \pm \infty$ inside the physical strip $-\frac{1}{2}<\operatorname{Im} x<\frac{1}{2}$;

\footnotetext{
${ }^{8}$ When $x \rightarrow \infty$ we know that $Y_{0}(x) \rightarrow e^{-L \cosh (\pi x)}$, i.e. it is exponentially small, as in the case of large $L$, and the Y-system, as well as the T-system splits in to two independent $u$ - and $v$-wings with $T_{0}(x) \sim \Phi(x) \sim \phi(x) \sim x^{N}$.
} 
The first property is somewhat similar to the forth property from the previous subsection: the large $x$ asymptotics is governed by the same exponential $e^{-L \cosh \pi \theta}$ as the large $L$ asymptotics. As a consequence of the second assumption, inspired by the integral representation (47) for the $U(1)$ sector, $T_{k>0}^{u}(x)$ are regular for $-(k+1) / 2<\operatorname{Im} x<(k+1) / 2$.

Similarly, for another solution we assume that

- $\frac{\bar{\Phi}_{v}(x)}{\phi(x+i / 2)}\left(\frac{\Phi_{v}(x)}{\phi(x-i / 2)}\right)$ is regular for $\operatorname{Im} x>-1 / 2(\operatorname{Im} x<1 / 2)$ in the all upper (lower) half plane and goes to 1 when $|x| \rightarrow \infty$ in all directions in the upper (lower) half plane

- $\frac{T_{0}^{v}(x)}{\phi(x)}$ is regular and goes to 1 at $x \rightarrow \pm \infty$ inside $-\frac{1}{2}<\operatorname{Im} x<\frac{1}{2}$ strip.

Note that with these properties all $Y_{k \neq 0}$ are automatically analytic in the physical strip, as we see from (18). $Y_{0}$ is, in the strict sense, only analytic on the real axis, but the detailed analysis of the Appendix D shows that we can expect its analyticity even in a finite strip around the real axis. As concerns the $T$-functions, although we use in different circumstances $T_{k}^{u}$ or $T_{k}^{v}$, we will get the same result for all $Y_{k}$, since they are related by a gauge transformation $g(x)$. But analyticity will be explicit only for one wing at a time: at $k \geq 1$ for $T_{k}^{u}$ and at $k \leq 1$ for $T_{k}^{v}$.

The listed properties are enough to explicitly relate $T_{k}^{u}, T_{k}^{v}$ with the corresponding $\Phi_{v}$ and $\Phi_{u}$ using a certain integral representation for them. In Appendix B we follow this line of arguments to formulate the complete set of equations for an arbitrary state, including a NLIE for $g(x)$ and the associated equations for the Bethe roots. However, it appears to be more advantageous, especially for the numerics, to use the integral representations for the logarithms of $T_{k}^{u}, T_{k}^{v}, \Phi_{v}$, $\Phi_{u}$. We will derive in what follows the corresponding equations defining the energy of a general state.

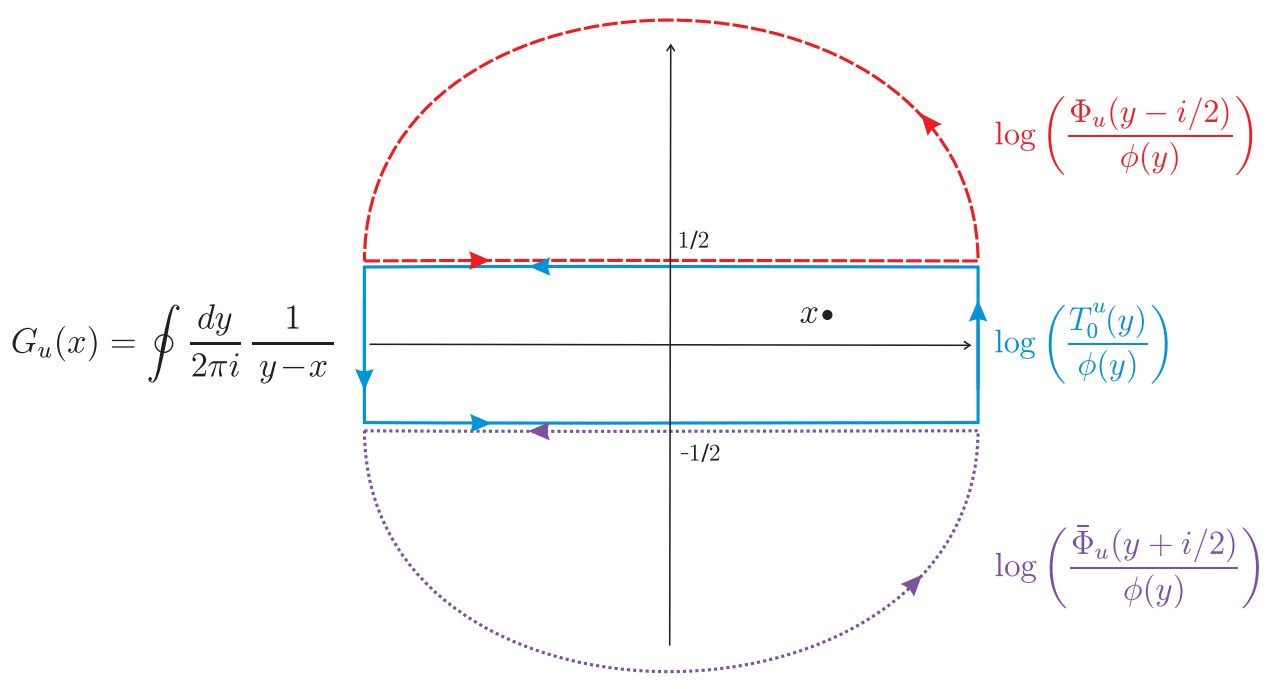

Figure 6: The ratios indicated close to each contour are analytic inside the corresponding contour. Thus we can obtain them in each of these regions using a single resolvent $G_{u}(x)$ as in (52).

Let us define two new functions analytic on a Riemann surface with two infinite cuts at $\operatorname{Im} x=$ $\pm i / 2$

$$
G_{w}(x)=\frac{1}{2 \pi i} \int_{-\infty}^{\infty} \frac{\rho_{w}(y)}{x-y-i / 2} d y-\frac{1}{2 \pi i} \int_{-\infty}^{\infty} \frac{\bar{\rho}_{w}(y)}{x-y+i / 2} d y, \quad(w=u, v)
$$


with the following spectral densities along the infinite cuts $^{9}$

$$
\rho_{u}(x)=\log \left(\frac{T_{0}^{u}(x+i / 2)}{\Phi_{u}(x)}\right), \quad \rho_{v}(x)=\log \left(\frac{T_{0}^{v}(x+i / 2)}{-\bar{\Phi}_{v}(x)}\right),
$$

and their complex conjugates

$$
\bar{\rho}_{u}(x)=\log \left(\frac{T_{0}^{u}(x-i / 2)}{\bar{\Phi}_{u}(x)}\right), \quad \bar{\rho}_{v}(x)=\log \left(\frac{T_{0}^{v}(x-i / 2)}{-\Phi_{v}(x)}\right) .
$$

Then from (18) we have

$$
\log \left(1+Y_{0}\right)=\rho_{u}+\bar{\rho}_{u}=\rho_{v}+\bar{\rho}_{v} .
$$

When $L$ is large enough we know from the results of the section 2.4 that $T_{0}^{w}(x) \simeq \Phi_{w}(x-i / 2) \simeq$ $\bar{\Phi}_{w}(x+i / 2) \simeq \phi(x)$ and thus to the leading order $\rho_{w}(x)$ 's are exponentially small. It is also important for our analyticity assumptions listed above to hold that $\rho_{w}(x) \sim e^{-L \cosh \pi x}$ at $x \rightarrow \pm \infty$ along the real axis. Together with these analyticity assumptions the following formulae are true at any $L$

$$
G_{v}(x)=\left\{\begin{array}{ll}
\log \frac{-\bar{\Phi}_{v}(x-i / 2)}{\phi(x)} & \operatorname{Im} x>+1 / 2 \\
\log \frac{T_{0}^{v}(x)}{\phi(x)} & |\operatorname{Im} x|<1 / 2 \\
\log \frac{-\Phi_{v}(x+i / 2)}{\phi(x)} & \operatorname{Im} x<-1 / 2
\end{array} \quad, \quad G_{u}(x)=\left\{\begin{array}{ll}
\log \frac{\Phi_{u}(x-i / 2)}{\phi(x)} & \operatorname{Im} x>+1 / 2 \\
\log \frac{T_{0}^{u}(x)}{\phi(x)} & |\operatorname{Im} x|<1 / 2 \\
\log \frac{\Phi_{u}(x+i / 2)}{\phi(x)} & \operatorname{Im} x<-1 / 2
\end{array} .\right.\right.
$$

These formulas are easily understood from simple contour manipulation as depicted in figure 6. Let us consider the resolvent $G_{u}$, plug (53) and (54) into (52) and consider separately the terms containing $\frac{T_{0}^{u}(x)}{\phi(x)}, \frac{\Phi_{u}(x-i / 2)}{\phi(x)}$ and $\frac{\bar{\Phi}_{u}(x+i / 2)}{\phi(x)}$. Since $\frac{T_{0}(x)}{\phi(x)} \rightarrow 1, x \rightarrow \pm \infty$, we can close the contour in the integrals containing $\frac{\log T_{0}(x)}{\phi(x)}$ around the physical strip and contracting it around the pole $y=x$ we obtain the middle relations in (56) if $x$ lies in the physical strip. Similarly, using the fact that in the upper half-plane $\frac{\Phi_{u}(x-i / 2)}{\phi(x)} \rightarrow 1, x \rightarrow \infty$, we can close the contour in the integrals containing $\log \frac{\Phi_{u}(x-i / 2)}{\phi(x)}$ (after the obvious shift of integration variable) around the upper half-plane and contracting it around the pole $y=x$ we obtain the upper relations in (56) provided $\operatorname{Im}(x)>1 / 2$. The integrals containing $\log \frac{\bar{\Phi}_{u}(x-i / 2)}{\phi(x)}$ are treated similarly with the contours being closed in the lower half-plane. For the resolvent $G_{v}$ the same sort of reasonings apply.

As we mentioned above, the two solutions of Hirota equation we defined in this way, are related by a gauge transformation $T_{k}^{v}=g \circ T_{k}^{u}$. However, the polynomials $Q_{u}$ and $Q_{v}$ are not necessarily related by this gauge transformation $g(x)$. Instead, one can easily see that $Q_{v}$ is mapped to another linearly independent solution of eq. (21) $R^{u}=\frac{Q^{v}}{g^{-}}$. We can use eqs. $(26,27)$ to express all $T_{k}$ 's and $\Phi$ 's in terms of $Q$ 's. In particular, we have

$$
\Phi_{u}=h^{+}\left(\frac{\mathcal{Q}_{u}^{++} \overline{\mathcal{Q}}_{v}^{--}}{g^{-}}-\frac{\mathcal{Q}_{u} \overline{\mathcal{Q}}_{v}}{g^{+}}\right), T_{0}^{u}=h\left(\frac{\mathcal{Q}_{u}^{+} \mathcal{Q}_{v}^{+}}{\bar{g}}-\frac{\overline{\mathcal{Q}}_{u}^{-} \overline{\mathcal{Q}}_{v}^{-}}{g}\right)
$$

\footnotetext{
${ }^{9}$ These spectral densities denoted by $\rho$ should not be confused with the densities of Bethe roots $\varrho$ used in appendix $\mathrm{A}$ in the derivation of the $\mathrm{Y}$-system ground state equations.
} 
Similar relations for $v$ wing can be obtained from the gauge transformation $\Phi_{v}=g^{-} g^{+} \Phi_{u}$ and $T_{0}^{v}=\bar{g} g T_{0}^{u}$. For the densities (53-54) this yields

$$
e^{\rho_{u}}=+\frac{T_{0}^{u+}}{\Phi_{u}}=\frac{\frac{g^{+}}{\bar{g}^{+}} \mathcal{Q}_{u}^{++} \mathcal{Q}_{v}^{++}-\overline{\mathcal{Q}}_{u} \overline{\mathcal{Q}}_{v}}{\frac{g^{+}}{g^{-}} \mathcal{Q}_{u}^{++} \overline{\mathcal{Q}}_{v}^{--}-\mathcal{Q}_{u} \overline{\mathcal{Q}}_{v}}, \quad e^{\rho_{v}}=-\frac{T_{0}^{v+}}{\bar{\Phi}_{v}}=\frac{\frac{g^{+}}{\bar{g}^{+}} \mathcal{Q}_{v}^{++} \mathcal{Q}_{u}^{++}-\overline{\mathcal{Q}}_{v} \overline{\mathcal{Q}}_{u}}{\frac{\bar{g}^{-}}{\bar{g}^{+}} \mathcal{Q}_{v}^{++} \overline{\mathcal{Q}}_{u}^{--}-\mathcal{Q}_{v} \overline{\mathcal{Q}}_{u}}
$$

Note that one can get $\rho_{v}$ from $\rho_{u}$ by exchanging indices $u \leftrightarrow v$ and $g \rightarrow 1 / \bar{g}$.

We see that the densities and thus both $G_{u}$ and $G_{v}$ now can be expressed solely in terms of three polynomials $\mathcal{Q}_{v}, \mathcal{Q}_{u}, \phi$ and a function $g(x)$, generating the gauge transformation relating the two wings. It is left only to find a closed equation on $g(x)$. We do it in the following subsection.

\subsubsection{Closed equation on the gauge function $g(x)$}

In the previous subsection, we managed to express all relevant quantities in terms of three polynomials $\mathcal{Q}_{v}, \mathcal{Q}_{u}, \phi$ and a complex function $g(x)$. Using the condition that two solutions of Hirota equation are related by the gauge transformation generated by $g(x)$ we can write a closed equation on that function. In particular, using the fact that $\Phi_{v}$ and $\Phi_{u}$ are related by the gauge transformations (25) we obtain

$$
\Phi_{v}=g^{+} g^{-} \Phi_{u} .
$$

It gives a closed relation on $g$ which we can rewrite, assuming that $g$ is regular within the physical strip, as follows

$$
g=i e^{\frac{1}{2} i L \sinh (\pi x)}\left(-\frac{\Phi_{v}}{\Phi_{u}}\right)^{* s},
$$

where the zero mode of the inverted operator was chosen to ensure the proper large $x$ asymptotic.

Indeed with this choice $T_{-1}^{u}=\frac{T_{-1}^{v}}{g^{-} \bar{g}^{+}} \sim e^{-L \cosh (\pi x)}$ leading to the right behavior of $Y_{0}$ (see eq.(18)) at large $L$. Using (52) this can be re-casted as

$$
g=i e^{\frac{1}{2} i L \sinh (\pi x)} S(x) \exp \left[s * G_{v}(x-i / 2-i 0)-s * G_{u}(x+i / 2+i 0)\right]
$$

where we used (56) and the identity

$$
\left(\frac{\phi^{-}}{\phi^{+}}\right)^{* s}=\left(S^{+} S^{-}\right)^{* s}=S
$$

following from the crossing relation (4). We remind that $\phi(x)=\prod_{j=1}^{N}\left(x-\theta_{j}\right)$ and $S(x)=$ $\prod_{j=1}^{N} S_{0}\left(x-\theta_{j}\right)$.

The closed NLIE (61) for $g(x)$ is our main result. Together with the expressions for the densities in terms of $g$ (53-54) it allows us to calculate $\left(1+Y_{0}\right)$ and thus to obtain the energy of a state (15).

In what follows in this section we will rewrite it in a little more convenient form which will be useful for the numerical computations for particular states.

Conjugating the last equation we find

$$
\bar{g}=-i e^{-i L / 2 \sinh (\pi x)} S^{-1}(x) \exp \left[s * G_{v}(x+i / 2+i 0)-s * G_{u}(x-i / 2-i 0)\right] .
$$

Finally it is useful to translate these equations into an equation for the phase $g / \bar{g}$

$$
\frac{g}{\bar{g}}=-e^{i L \sinh (\pi x)} S^{2}(x) \exp \left(\frac{1}{2}\left[K_{0}^{-} *\left(\rho_{u}+\rho_{v}\right)-K_{0}^{+} *\left(\bar{\rho}_{u}+\bar{\rho}_{v}\right)\right]\right),
$$


where $K_{0}=\frac{1}{2 \pi i} \partial_{x} \log S_{0}^{2}$ and we used

$$
G_{w}(x+i / 2+i 0)-G_{w}(x-i / 2-i 0)=K_{1}(x+i / 2-i 0) * \bar{\rho}_{w}-K_{1}(x-i / 2+i 0) * \rho_{w}
$$

and the convolution form of the dressing kernel as $K_{0}=2 s * K_{1}, K_{1}(x)=\frac{2}{\pi\left(4 x^{2}+1\right)}$. For the squared norm $g \bar{g}$ we get from eq.(60)

$$
g \bar{g}=\left(\frac{\Phi_{v}}{\Phi_{u}} \frac{\bar{\Phi}_{v}}{\bar{\Phi}_{u}}\right)^{* s}=\left[\frac{\Phi_{v} \bar{\Phi}_{v}}{T_{0}^{v+} T_{0}^{v-}} \frac{T_{0}^{u+} T_{0}^{u-}}{\Phi_{u} \bar{\Phi}_{u}}\right]^{* s} \frac{T_{0}^{v}}{T_{0}^{u}}=\exp \left(G_{v}-G_{u}\right),
$$

where we used $\frac{1}{Y_{0}} Y_{0}=1$ inside the square brackets to get the last equality. This equation can be also obtained from the gauge transformation $T_{0}^{v}=g \bar{g} T_{0}^{u}$. As we shell see in section 5 these equations can be efficiently solved numerically, by iteration, where at each iteration step a single convolution integral arises involving the densities $\rho_{u}$ and $\rho_{v}$.

We use eq.(58) and eq.(59) together with our analyticity assumptions to constrain $g, \Phi_{u, v}$ and $T_{0}^{u, v}$. In the next section we will fix the remaining finite number of complex parameters - zeros of polynomials $\mathcal{Q}_{v}, \mathcal{Q}_{u}$ and the real zeroes of $\phi$. After that one can use eq.(23) to construct all $T_{k}$. In appendix $\mathrm{C}$ we show that all $T_{k}$ obtained in this way will be real functions and thus Hirota equation for them is satisfied. This means that we solved indeed the Y-system with the right physical analytic properties for the solutions.

\subsubsection{Finite size Bethe equations and the energy}

Finally it is left to explain how to fix the finite number of constants, the Bethe roots $\theta_{j}, u_{j}$ and $v_{j}$, which are zeros of the polynomials $\phi, \mathcal{Q}_{u}, \mathcal{Q}_{v}$ and which completely characterize a state. The zeros of $\phi$ are by definition the zeros of $T_{0}$ which means that at these points $e^{\rho_{u}\left(\theta_{j} \pm i / 2\right)}=e^{\rho_{v}\left(\theta_{j} \pm i / 2\right)}=0$ as we can see from eq.(58). It can be also written as follows

$$
\frac{\mathcal{Q}_{u}^{+} \mathcal{Q}_{v}^{+}}{\overline{\mathcal{Q}}_{u}^{-} \overline{\mathcal{Q}}_{v}^{-}} \frac{g}{\bar{g}}=1, \quad \text { at } x=\theta_{j},
$$

which we can rewrite using eq.(64) as

$$
e^{-i L \sinh \left(\pi \theta_{j}\right)}=-S^{2}\left(\theta_{j}\right) \frac{\mathcal{Q}_{u}^{+} \mathcal{Q}_{v}^{+}}{\overline{\mathcal{Q}}_{u}^{-} \overline{\mathcal{Q}}_{v}^{-}} \exp \left(\frac{1}{2}\left[K_{0}^{-} *\left(\rho_{u}+\rho_{v}\right)-K_{0}^{+} *\left(\bar{\rho}_{u}+\bar{\rho}_{v}\right)\right]\right), \quad \text { at } x=\theta_{j} .
$$

Note that when $L \rightarrow \infty$ we can neglect the last factor to get precisely the usual infinite volume ABA eq.(7). The equations for the auxiliary Bethe roots $u_{j}$ can be derived in many alternative ways. The most standard way is to demand analyticity of $T_{1}$ at $x=u_{j}$ (see eq.(22))

$$
\Phi_{u} \overline{\mathcal{Q}}_{u}^{--}+T_{0}^{u-} \mathcal{Q}_{u}^{++}=0, \text { at } x=u_{j} .
$$

We see that in general there is no reason to assume $u_{j}$ to be real when $L$ is finite. Using the resolvent $G_{u}$ to represent $T_{0}$ and $\Phi_{u}$ appearing in this expression we get the auxiliary Bethe equations following from (69) in the form

$$
1=-\frac{\phi^{-} \mathcal{Q}_{u}^{++}}{\phi^{+} \overline{\mathcal{Q}}_{u}^{--}} P\left(u_{j}\right)
$$

where $P(x)$ is defined on the upper half plane by

$$
P(x)=\exp \left[K_{1}(x-i / 2) * \rho_{u}-K_{1}(x+i / 2) * \bar{\rho}_{u}\right], \quad \operatorname{Im} x>0,
$$


In the large $L$ limit $P(x) \sim 1$ and we get the ABA eq.(8). A similar equation fixes the roots $v_{j}$.

The integral equation (61) together with the equations (68), (70) fixing the zeros of the polynomials $\mathcal{Q}_{u}, \mathcal{Q}_{v}, \phi$ are the complete set of equations which one should solve to find the full spectrum of the $S U(2) \times S U(2)$ principal chiral field. Once $g(x)$ and the positions of the zeros $\theta_{j}, u_{j}, v_{j}$ are found, we can compute the exact energy of the corresponding quantum state from eqs. $(15,55)$ and $(58)$

$$
E=\sum_{k=1}^{N} \cosh \left(\pi \theta_{k}\right)-\frac{1}{2} \int \cosh (\pi x)\left(\rho_{u}+\bar{\rho}_{u}\right) d x
$$

where we can use due to (58) $\rho_{u}+\bar{\rho}_{u}=\rho_{v}+\bar{\rho}_{v}=\log \left|\frac{\frac{g^{+}}{\bar{g}^{+}} \mathcal{Q}_{u}^{++} \mathcal{Q}_{v}^{++}-\overline{\mathcal{Q}}_{u} \overline{\mathcal{Q}}_{v}}{\frac{g^{+}}{g^{-}} \mathcal{Q}_{u}^{++} \overline{\mathcal{Q}}_{v}^{--}-\mathcal{Q}_{u} \overline{\mathcal{Q}}_{v}}\right|$.

Let us remark that our construction for a general state in this paper was based on the assumption that in the asymptotic regime $L \rightarrow \infty$ all the roots $u_{j}, v_{j}$ become real.However, it is well known that the complex solutions are also possible. We hope that even in this case our equations maintain their form, although this situation deserves a special care.

In section 4 we will explain how to efficiently implement these equations for numerical study. Before that, in the next section we will study the large $L$ behavior of these equations thus reproducing not only the large volume results of section 2.4 but also the subleading corrections (Lüscher corrections).

\subsection{Large volume limit: ABA and Lüscher corrections}

The $S U(2)$ principal chiral field spectrum is given by (15), or (72). As we have seen in the previous section, in the large $L$ limit the Bethe roots $\theta_{j}$ are given by their asymptotic values obtained from a solution to the asymptotic Bethe equations, and since $Y_{0}$ and $\rho$ 's are exponentially small we can drop the integral contribution in (68) and (70) and recover the usual asymptotic spectrum. In this section we focus on the leading finite size corrections to this result.

Due to these corrections auxiliary roots $u_{j}$ and $v_{j}$ become complex even if they were real asymptotically at large $L$. In this section we denote the real part of the roots $u_{j}, v_{j}$ by $U_{j}$ and $V_{j}$ while the (small) imaginary parts we denote by $\Delta u_{j}$ and $\Delta v_{j}$. The positions of the momentum carrying roots $\theta_{j}$ are also corrected, however they stay real. We will also use the notation

$$
\mathfrak{Q}_{u}(x)=\prod_{j}\left(x-U_{j}\right), \mathfrak{Q}_{v}(x)=\prod_{j}\left(x-V_{j}\right) .
$$

We have to compute the first correction to the positions of the Bethe roots. To the leading order we can drop the exponentially small densities $\rho_{u}$ and $\rho_{v}$ in eq.(61), to get

$$
g(x) \simeq i S(x) e^{i L / 2 \sinh (\pi x)}
$$

which we can use to compute the spectral densities from (53-54). We see that some terms in the expression for $\rho_{u}$ are exponentially suppressed and we can expand

$$
\rho_{u} \simeq \frac{\overline{\mathcal{Q}}_{u}-\mathcal{Q}_{u}}{\mathcal{Q}_{u}}+e^{-L \cosh (\pi x)} \frac{S^{+}}{S^{-}} \frac{\mathcal{Q}_{u}^{++}}{\phi^{+}} \frac{\mathcal{Q}_{v}^{++} \phi^{-}+\mathcal{Q}_{v}^{--} \phi^{+}}{\mathcal{Q}_{v} \mathcal{Q}_{u}} \simeq \frac{\overline{\mathcal{Q}}_{u}-\mathcal{Q}_{u}}{\mathfrak{Q}_{u}}+\frac{\mathfrak{Q}_{u}^{++} T_{-1}^{u}}{\mathfrak{Q}_{u} \phi^{+}}
$$


In the last equality we neglect the small imaginary part of the axillary roots and we use (22) to the leading order together with the gauge transformation $T_{-1}^{v}=g^{-} \bar{g}^{+} T_{-1}^{u}$ and the crossing relation $S^{+} S^{-}=\frac{\phi^{-}}{\phi^{+}}$.

The poles at $x=U_{j}$ should cancel, due to eq.(70), among the first and the second term since the density by definition is regular. We introduce the notations $\rho_{u}^{(1)}$ and $\rho_{u}^{(2)}$ for the first and the second term in (75). The first one can be simply written as

$$
\rho_{u}^{(1)} \simeq \sum_{j} \frac{2 \Delta u_{j}}{x-U_{j}}
$$

Since the whole density is regular we can apply the principal part prescription to the finite integrals in (64) without changing the result. Having done so we are free to split the convolutions into convolutions with $\rho^{(1)}$ and $\rho^{(2)}$. In (68) we should then expand the factor

$$
\left[\frac{\mathcal{Q}_{u}^{+}}{\overline{\mathcal{Q}}_{u}^{-}} \exp \left(i \operatorname{Im} f K_{0}^{-}(x-y) \rho_{u}^{(1)}(y)\right)\right] \exp \left(i \operatorname{Im} f K_{0}^{-}(x-y) \rho_{u}^{(2)}(y)\right)
$$

and the similar factor for the $v$ roots, to the next to leading order. We notice that $\rho_{u}^{(1)}$ is purely imaginary to the leading order, as seen from eq.(76), and therefore we can simplify the term in the square brackets

$$
\frac{\mathcal{Q}_{u}^{+}}{\overline{\mathcal{Q}}_{u}^{-}} \exp \left(\frac{1}{2}\left(K_{0}^{-}+K_{0}^{+}\right) * \rho_{u}^{(1)}\right) \simeq \frac{\mathcal{Q}_{u}^{+}}{\overline{\mathcal{Q}}_{u}^{-}}\left(1+K_{1} * \rho_{u}^{(1)}\right)=\frac{\mathcal{Q}_{u}^{+}}{\overline{\mathcal{Q}}_{u}^{-}}\left(1+\frac{\rho_{u}^{(1)+}+\rho_{u}^{(1)-}}{2}\right) \simeq \frac{\mathfrak{Q}_{u}^{+}}{\mathfrak{Q}_{u}^{-}}
$$

where the convolutions are understood in the sense of principal value. Thus in the Bethe equations (67) in this approximation the imaginary parts of the axillary roots cancel against the contribution from $\rho^{(1)}$ and we simply get

$$
-e^{i L \sinh (\pi x)} S^{2} \frac{\mathfrak{Q}_{u}^{+} \mathfrak{Q}_{v}^{+}}{\mathfrak{Q}_{u}^{-} \mathfrak{Q}_{v}^{-}}=\exp \left(-i \operatorname{Im} K_{0}^{-} *\left[\rho_{u}^{(2)}+\rho_{v}^{(2)}\right]\right) \text { at } x=\theta_{j} .
$$

Proceeding in the same fashion in the eq.(70) for the auxiliary Bethe roots we arrive at a similar conclusion. Namely only the real parts of the auxiliary roots survive when we separate the density into $\rho^{(1)}$ and $\rho^{(2)}$

$$
-\frac{\phi^{-}}{\phi^{+}} \frac{\mathfrak{Q}_{u}^{++}}{\mathfrak{Q}_{u}^{--}}=\exp \left(-2 i \operatorname{Im} K_{1}^{-} * \rho_{u}^{(2)}\right) \text { at } x=U_{j} .
$$

See appendix E for details. We see that all terms except for the convolutions with $\rho^{(2)}$ have a simple effect of absorbing the imaginary parts of the Bethe roots.

It turns out that the remaining convolutions, containing $\rho^{(2)}$, can be nicely written in terms of the leading order $Y_{0}$ found before in (32),

$$
Y_{0}(x) \simeq e^{-L \cosh (\pi x)}\left(\frac{\mathcal{Q}_{u}^{++} \phi^{-}+\mathcal{Q}_{u}^{--} \phi^{+}}{\mathcal{Q}_{u}}\right)\left(\frac{\mathcal{Q}_{v}^{++} \phi^{-}+\mathcal{Q}_{v}^{--} \phi^{+}}{\mathcal{Q}_{v}}\right) \frac{\left(S^{+}\right)^{2}}{\left(\phi^{-}\right)^{2}}
$$

where the $\theta_{j}$ appear in $\phi$ and $S$ while the $u_{j}\left(v_{j}\right)$ auxiliary roots appear in the corresponding Baxter polynomials $\mathcal{Q}_{u}\left(\mathcal{Q}_{v}\right)$. Notice that this quantity is already exponentially small, so we can take here the asymptotic values for the auxiliary roots. As explained in detail in appendix E, the quantities inside the principal part integrals are related to the derivative of this function with respect to $\theta_{k}$ or 
$u_{k}$ and $v_{k}$ which we treat in (81) as independent variables. More precisely we have the remarkable identities

$$
\begin{aligned}
i \operatorname{Im}\left(K_{0}^{-}\left(\theta_{i}-y\right)\left[\rho_{u}^{(2)}(y)+\rho_{v}^{(2)}(y)\right]\right) & =-\frac{\partial_{\theta_{i}} Y_{0}(y)}{2 \pi i} \\
2 i \operatorname{Im}\left(K_{1}^{-}\left(u_{j}-y\right) \rho_{u}^{(2)}(y)\right) & =+\frac{\partial_{u_{i}} Y_{0}(y)}{2 \pi i}
\end{aligned}
$$

Thus we finally obtain the corrected Bethe ansatz equations in the following elegant form:

$$
\begin{aligned}
-\frac{\phi^{+}}{\phi^{-}} \frac{\mathfrak{Q}_{u}^{--}}{\mathfrak{Q}_{u}^{++}} & =\exp \left(f \frac{\partial_{U_{j}} Y_{0}(y)}{2 \pi i} d y\right) \text { at } x=U_{j}, \\
-e^{i L \sinh (\pi x)} S^{2} \frac{\mathfrak{Q}_{u}^{+} \mathfrak{Q}_{v}^{+}}{\mathfrak{Q}_{u}^{-} \mathfrak{Q}_{v}^{-}} & =\exp \left(f \frac{\partial_{\theta_{j}} Y_{0}(y)}{2 \pi i} d y\right) \text { at } x=\theta_{j}, \\
-\frac{\phi^{+}}{\phi^{-}} \frac{\mathfrak{Q}_{v}^{--}}{\mathfrak{Q}_{v}^{++}} & =\exp \left(f \frac{\partial_{V_{j}} Y_{0}(y)}{2 \pi i} d y\right) \text { at } x=V_{j} .
\end{aligned}
$$

It is not completely surprising that we managed to express everything in terms of $Y_{0}$. To the leading order, $Y_{0}$ can be expressed in terms of S-matrix only: it is the relevant eigenvalue of the operator

$$
e^{-L \cosh (\pi x)} \operatorname{tr}\left(\hat{S}_{01}\left(x-\theta_{1}\right) \hat{S}_{02}\left(x-\theta_{2}\right) \ldots \hat{S}_{0 N}\left(x-\theta_{N}\right)\right) .
$$

We see that (84) corresponds precisely to the conjectured equation (27) in [5] only inside the $U(1)$ sector. However, our result is different from outside the $U(1)$ sector when there are axillary roots $U_{j}$ and $V_{j}$. Finally the equation for the energy of the state corrected by the finite size effects is given by eq.(15) in terms of $Y_{0}$ in the leading approximation and the corrected positions of the roots $\theta_{j}$ which should be found from eq.(84).

The right-hand sides of the corrected Bethe equations (84) have a simple interpretation: for the middle equation, it reflects the contribution of scattering of the "physical" particles off the virtual ones on the cylinder, whether as the other two reflect the same effect for the "magnons" responsible for the isotopic degrees of freedom of the particles. Although these equations are derived here only for a particular model their form looks very universal and can be immediately generalized to any other integrable sigma model where the exact scattering matrix is known.

\subsubsection{Single particle case}

In this section we consider the single particle case for the Lüscher-type correction of the previous subsections. This analysis was done in a more general context in [5].

When we have a single particle with momentum $\theta_{1}$ (84) yields simply

$$
L \sinh \left(\pi \theta_{1}\right)=2 \pi n-f \frac{d y}{2 \pi} \partial_{\theta_{1}} Y_{0}(y),
$$

which corrects the leading order quantization condition

$$
L \sinh \left(\pi \theta_{1}^{0}\right)=2 \pi n
$$


Now, from (81) we see that the $x$ dependence in $Y_{0}(x)$ comes from the exponential factor $e^{-L \pi \cosh (\pi x)}$ and also from the combinations $x-\theta_{j}, x-u_{j}$ and $x-v_{j}$ appearing in the remaining terms in this expression. Thus

$$
\partial_{y} Y_{0}(y)=-L \pi \sinh (\pi y) Y_{0}(y)-\sum_{k=1}^{N} \partial_{\theta_{k}} Y_{0}(y)-\sum_{k=1}^{J_{u}} \partial_{u_{k}} Y_{0}(y)-\sum_{k=1}^{J_{v}} \partial_{v_{k}} Y_{0}(y),
$$

which, in the case we are considering, with $N=1$ and $J_{u}=J_{v}=0$, allows us to simplify (86) to

$$
L \sinh \left(\pi \theta_{1}\right)=2 \pi n+\int \frac{d y}{2} L \sinh (\pi y) Y_{0}(y)
$$

so that the leading finite size correction to the energy (15) reads

$$
E(L)-\cosh \left(\pi \theta_{1}^{0}\right) \simeq-\frac{1}{2} \int \cosh (\pi y)\left(1-\tanh (\pi y) \tanh \left(\pi \theta_{1}^{0}\right)\right) e^{-L \cosh (\pi y)} \operatorname{tr} \hat{S}_{01}\left(y-\theta_{1}^{0}\right),
$$

precisely as expected for the Lüscher corrections [4].

\section{$4 S U(2)$ Chiral Gross-Neveu model and related models}

Our NLIE resembles the Destri-deVega equation and, at least in the cases the last one is known, can even coincide with it. In the cases when the DdV equation is not known, like the $S U(2)_{L} \times S U(2)_{R}$ $\mathrm{PCF}$, or $\mathrm{O}(4)$ model studied in this paper, we obtain a new, DdV-like equation. In this subsection, to demonstrate our method, we show how to reproduce the DdV equation for the chiral $S U(2)$ Gross-Neveu model on a finite circle.

The TBA equations for this model are given by the same $Y$-system (12) with an important difference that $Y_{s<0}=0$ (see Fig.8). In particular, since $Y_{-1}=0$ we have $T_{-2}=0$ which implies due to the eqs. $(21,17)$

$$
0=T_{-1}^{--} \bar{Q}-\bar{\Phi} Q^{--}, T_{-1}^{+} T_{-1}^{-}=\Phi^{-} \bar{\Phi}^{+}
$$

Then it is immediate to check that the quantity

$$
\mathcal{A} \equiv \frac{Q^{+}}{\bar{Q}^{-}} \frac{T_{-1}^{-}}{\Phi^{-}}=\frac{Q^{+}}{\bar{Q}^{-}} \frac{\bar{\Phi}^{+}}{T_{-1}^{+}}
$$

satisfies, due to eq.(91), the relation

$$
\mathcal{A}^{+} \mathcal{A}^{-}=\left(\frac{Q^{+}}{\bar{Q}^{-}}\right)^{+}\left(\frac{Q^{+}}{\bar{Q}^{-}}\right)^{-} \frac{\bar{\Phi}}{\Phi} .
$$

Note that $\mathcal{A}$ is a pure phase on the real axe. Thus, restoring the proper zero mode exponential, we find

$$
\mathcal{A}=e^{i L \sinh (\pi x)} \frac{Q^{+}}{\bar{Q}^{-}}\left(\frac{\bar{\Phi}}{\Phi}\right)^{* s}
$$

As before, to make this a closed equation on $\mathcal{A}$ we introduce the resolvent $G$ and the density $\rho$

$$
G(x)=\frac{1}{2 \pi i} \int \frac{\rho(y)}{x-y-i / 2} d y-\frac{1}{2 \pi i} \int \frac{\bar{\rho}(y)}{x-y+i / 2} d y \quad, \quad \rho=\log \frac{T_{0}^{+}}{\Phi} .
$$


Again analytic properties of $T_{0}$ and $\Phi$ lead to eq.(56). Using the linear problem (21) we can write $T_{-1} Q^{++}-T_{0}^{+} Q=-\Phi \bar{Q}$ and we see that

$$
\rho=\log \left[\frac{\bar{Q}}{Q}\left(1+\mathcal{A}^{+}\right)\right] .
$$

Thus $\mathcal{A}$ satisfies the closed equation for $\mathcal{A}$ since $\Phi=G(x+i / 2+i 0)$. We see that $\mathcal{A}(x)$ plays a similar role as $g(x)$ in PCF. We can easily compute $Y_{0}$ in terms of $\mathcal{A}$

$$
1+Y_{0}=e^{\rho} e^{\bar{\rho}}=\left(1+\mathcal{A}^{+}\right)\left(1+1 / \mathcal{A}^{-}\right) .
$$

We see that the factors of $Q$ cancel from this expression. It is also possible to write eq.(94) in a simpler form without factors of $Q$ using the useful identities. First using eq.(65) and assuming that the density is regular not only on the real axes but also for $-1 / 2<\operatorname{Im} x \leq 0$ we write

$$
\left(\frac{\bar{\Phi}}{\Phi}\right)^{* s}=S_{\theta} \exp \left(\frac{1}{2} K_{0}^{-} * \rho-\frac{1}{2} K_{0}^{+} * \bar{\rho}\right)=S_{\theta} \exp \left(\frac{1}{2} K_{0} *\left[\rho^{-+}-\bar{\rho}^{+-}\right]\right)
$$

where $\rho^{-+} \equiv \rho(x-i / 2+i 0)$. Using the following relation

$$
\frac{1}{2} K_{0} * \log \left[\frac{\bar{Q}^{+} \bar{Q}^{-}}{Q^{-} Q^{+}}\right]=\log \left[\frac{S_{u}^{+}}{S_{\bar{u}}^{+}} \frac{\bar{Q}^{-}}{Q^{-}}\right]
$$

where

$$
S_{\theta}(x)=\prod_{j=1}^{N} S_{0}\left(x-\theta_{j}\right), S_{u}(x)=\prod_{j=1}^{J_{u}} S_{0}\left(x-u_{j}\right), S_{\bar{u}}(x)=\prod_{j=1}^{J_{u}} S_{0}\left(x-\bar{u}_{j}\right)
$$

we can get rid of $Q$ 's in (98) and finally obtain the known DdV equation

$$
\mathcal{A}=e^{i L \sinh (\pi x)} \frac{S_{\theta}}{S_{\bar{u}}^{+} S_{u}^{-}} \exp \left(i \operatorname{Im} K_{0} * \log [1+\mathcal{A}(x+i 0)]\right) .
$$

Then for $Y_{0}$ we get the standard relation which should be used to compute the exact spectrum from

$$
E(L)=-\frac{1}{2} \int m \cosh (\pi \theta) \log \left(1+Y_{0}\right)+\sum m \cosh \left(\pi \theta_{j}\right) .
$$

Furthermore, both BAE - for the physical rapidities $\theta_{j}$ and for the magnon rapidities $u_{j}-$ can be written

$$
\mathcal{A}\left(\theta_{j}\right)=-1, \mathcal{A}^{+}\left(u_{j}\right)=-1 .
$$

We also notice that $\mathcal{A}(x)$ has poles at $x=\bar{u}_{j}+i / 2$. To see that we use

$$
\begin{aligned}
& T_{0}\left(\theta_{j}\right)=0 \Rightarrow 1+\mathcal{A}\left(\theta_{j}\right)=\frac{Q^{-}\left(\theta_{j}\right)}{\bar{Q}^{-}\left(\theta_{j}\right)} \frac{T_{0}\left(\theta_{j}\right)}{\Phi^{-}\left(\theta_{j}\right)}=0, \\
& \Phi\left(u_{j}\right) \sim 1 \Rightarrow 1+\mathcal{A}^{+}\left(u_{j}\right)=\frac{Q\left(u_{j}\right)}{\bar{Q}\left(u_{j}\right)} \frac{T_{0}^{+}\left(u_{j}\right)}{\Phi\left(u_{j}\right)}=0 .
\end{aligned}
$$

Equations (100),(97),(102) and (103) are precisely the DdV equations derived in $[6,7]^{10 !}$ As shown in this section our method can be directly generalized to other models whose TBA $Y$-system

\footnotetext{
${ }^{10}$ In these papers the sine-Gordon model was considered. The $S U(2)$ Chiral Gross-Neveu model is a simple limiting case of this theory, see discussion in the paragraph below.
} 
equations are known. It would be very interesting to make a systematic study of such models using our formalism.

For example, a simple generalization of the case studied in this section is obtained by considering the functions $T_{k}, \Phi$ as well as the Baxter functions $Q$ to be periodic in the imaginary directions with period $i \nu$. This amounts to considering the trigonometric solutions of Hirota equation (17) and the corresponding linear problem (21) - this should correspond to the sine-Gordon model [13]. We take the Baxter polynomials $Q(x)$ and the large $L$ limit of $T_{0}, \Phi^{-}, \bar{\Phi} \simeq \tilde{\phi}$ to be

$$
\tilde{Q}(x)=\prod_{j=1}^{J} \frac{\sinh \frac{\pi}{\nu}\left(x-u_{j}\right)}{\sinh \frac{\pi}{\nu}}, \tilde{\phi}(x)=\prod_{j=1}^{N} \frac{\sinh \frac{\pi}{\nu}\left(x-\theta_{j}\right)}{\sinh \frac{\pi}{\nu}}
$$

instead of polynomials. Then most of the formulae in this section go through with minor modifi-

cations. For example, instead of the $S U(2)$ Chiral Gross-Neveu S-matrix $S_{0}=\left(\frac{x-i / 2}{x+i / 2}\right)^{* s}$ we will find the sine-Gordon dressing factor

$$
\tilde{S}_{0}=\left(\frac{\sinh \frac{\pi}{\nu}(x-i / 2)}{\sinh \frac{\pi}{\nu}(x+i / 2)}\right)^{* s}=-i \exp \int_{0}^{\infty} \frac{\sin (\omega x)}{\omega} \frac{\sinh \left(\frac{\nu-1}{2} \omega\right)}{\cosh \left(\frac{\omega}{2}\right) \sinh \left(\frac{\nu}{2} \omega\right)} .
$$

Thus it seems that our method allows to derive the sine-Gordon DdV equations of $[6,7]$ in an easy way. For $\nu \rightarrow \infty$ we recover the $S U(2)$ chiral Gross-Neveu model. For an integer $\nu$ the Y-system can be truncated as represented in figure 8, see e.g. [13].

Another interesting class of models which one could analyze using our formalism is represented by the so called sausage model (see e.g. [45]). This model can be considered as a generalization of the $O(4)$ model, or $S U(2) \mathrm{PCF}$, in the same sense as the sine-Gordon model is a generalization of the $S U(2)$ chiral Gross-Neveu. The inhomogeneous XXX-spin chain present in the $S U(2)$ GrossNeveu model and describing the isotopic degrees of freedom is generalized in sine-Gordon model to the XXZ chain, with the anisotropy parameter $\nu$ introduced above. Similarly, the sausage models can be seen as two interacting inhomogeneous XXZ chains parameterized by the inhomogeneities $\theta_{1}, \ldots, \theta_{N}$ and anisotropies $\nu$ and $\nu^{\prime}$. It would be very interesting to generalize our $O(4)$ model results to this more general class of models.

Our approach to deriving DdV like equations is strongly based on a smooth interpolation starting from the IR asymptotic Bethe ansatz description; hence, by construction, our states are very well identified. On the other hand we did not carry out a detailed study of complex solutions such as the states represented by Bethe strings in the large $L$ limit; for these states some of our formulae might need to be modified. Within the DdV approach based on descritizations of integrable models, many interesting complex solutions were studied: e.g. holes, special objects, wide roots, self-conjugate roots etc. It would be interesting to complete our approach to include all physical complex solutions and thus obtain a precise dictionary between these two approaches. In particular this would teach us which solutions to DdV NLIE correspond to physical states.

\section{$5 \quad$ Numerics}

In this section we explain how to efficiently solve numerically the equations derived in the Sec.3. 


\subsection{Implementation of numerics and Mathematica code}

For simplicity let us focus on the $U(1)$ sector where we consider $M$-particle quantum states with $M$ spins pointing in the same direction in $S U(2)_{L}$ and $S U(2)_{R}$. Then our equations simplify considerably as was explained in subsection 2.7. First of all, since there are no spins excited we have trivial Baxter polynomials $\mathcal{Q}_{u}=\mathcal{Q}_{v}=1$. Thus, from (58) we see that $\rho_{u}=\rho_{v}=\rho$ with

$$
\rho=\log \frac{\frac{g^{+}}{\bar{g}^{+}}-1}{\frac{g^{+}}{g^{-}}-1} .
$$

We also notice that since this is a symmetric configuration where the $u$ and $v$ root configurations are the same (there are no roots at all) we have, see e.g. (66), $g \bar{g}=1$ and thus $g(x)$ is a pure phase. In particular, for real $x$, we can simplify the density to

$$
\rho(x)=\log \frac{\left(g^{+}\right)^{2}-1}{\left|g^{+}\right|^{2}-1},
$$

from where we see that we can express it solely in terms of $g^{+}$. Since $g$ is a pure phase we need only to determine its argument from (64) which now reads

$$
g^{2}=-e^{i L \sinh (\pi x)} S^{2}(x) \exp \left(2 i \operatorname{Im}\left[K_{0}^{-} * \log \frac{\left(g^{+}\right)^{2}-1}{\left|g^{+}\right|^{2}-1}\right]\right) .
$$

This is almost perfect for numerical implementation but still needs to be slightly improved. The reason is that we want to iterate this equation by evaluating the right hand side for real $x$. But this will yield the updated values of $g(x)$ in the left hand side whereas for the next iteration we would need $g^{+}(x)$. To fix it, we simply shift $x \rightarrow x+i / 2$ in this equation and define $A(x) \equiv\left(g^{+}(x)\right)^{2}$ to get

$$
A=-e^{-L \cosh (\pi x)} \prod_{j=1}^{M} S_{0}^{2}\left(x-\theta_{j}+i / 2\right) \exp \left(K_{0} * \log \frac{A-1}{|A|-1}-K_{0}^{++} * \log \frac{\bar{A}-1}{|A|-1}-\log \frac{\bar{A}-1}{|A|-1}\right),
$$

where the convolution of $K_{0}^{++}$is understood in the principal part sense. We have explicitly written $S(x)$ to render the presence of the Bethe rapidities more explicit. These are fixed by the main Bethe equation (67) which in our notations is simply

$$
-e^{i L \sinh (\pi x)} \prod_{j=1}^{M} S_{0}^{2}\left(x-\theta_{j}\right) \exp \left(2 i \operatorname{Im}\left[K_{0}^{-} * \log \frac{A-1}{|A|-1}\right]\right)=1, x=\theta_{i} .
$$

For completeness let us present here the Mathematica code to solve these two equations by iterations $^{11}$. It is a slightly simplified, and thus less efficient, version of the code we used for the plots in figure 2 .

First we introduce the S-matrix $S_{0}$ and the kernel $K_{0}$,

$$
\begin{aligned}
& \mathrm{S} 0\left[\mathrm{x}_{-}\right]=\mathrm{I} * \operatorname{Gamma}[-(\mathrm{x} /(2 \mathrm{I}))] \operatorname{Gamma}[1 / 2+\mathrm{x} /(2 \mathrm{I})] /(\operatorname{Gamma}[\mathrm{x} /(2 \mathrm{I})] \operatorname{Gamma}[1 / 2-\mathrm{x} /(2 \mathrm{I})]) ; \\
& \mathrm{KO}\left[\mathrm{x}_{-}\right]=\mathrm{D}\left[\log \left[\mathrm{SO}[\mathrm{x}]^{\wedge} 2\right], \mathrm{x}\right] /(2 * \operatorname{Pi} * \mathrm{I}) ;
\end{aligned}
$$

\footnotetext{
${ }^{11}$ One can copy the code directly to Mathematica from .pdf
} 
Next we specify the size $L$ and the mode numbers $n=\left\{n_{1}, \ldots, n_{M}\right\}$. For example, if we want to study the system with $L=1 / 2$ and three particles with zero mode numbers we write

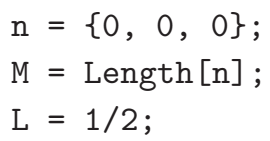

We will perform several integrals from $-\infty$ to $+\infty$ but the integrands have exponential tails so that it is quite useful to introduce a cut-off $X$ for all the integration intervals at this point. A reasonable cut-off is given by $e^{-L \cosh (\pi X)}=10^{-8}$. Furthermore, at each iteration step we will have to construct an updated function $A(x)$ which we do by means of an interpolation function,

$\mathrm{X}=\operatorname{ArcCosh}[8 \log [10] / \mathrm{L}] /[\mathrm{Pi}]$;

$\mathrm{F}\left[\mathrm{S}_{-}\right]:=$FunctionInterpolation $[\mathrm{S},\{\mathrm{x},-\mathrm{X}, \mathrm{X}\}$, InterpolationPoints->30] ;

Next we introduce

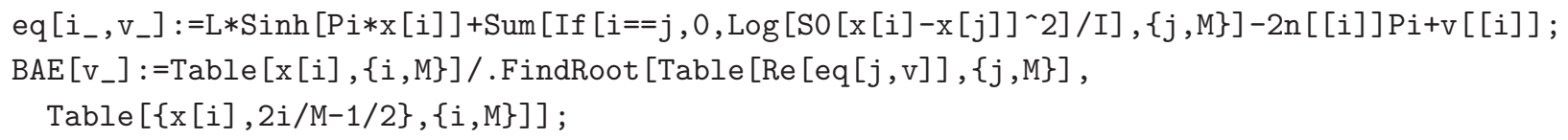

which yields the solution to Bethe equations $L \sinh \left(\pi \theta_{i}\right)+\sum_{j \neq i} \frac{1}{i} \log S_{0}^{2}\left(\theta_{i}-\theta_{j}\right)-2 \pi n_{i}+v_{i}$ where $v_{i}$ is a perturbation to the equation number $i$. Comparing with (111) we see that this perturbation at step $k$ will be given by the convolution appearing in (111) evaluated at the solution $\theta_{i}$ computed in the previous step. The leading order BAE's correspond to $v_{i}=0$ and are thus given by

$\backslash[$ Theta $][0]=\operatorname{BAE}[$ Table $[0,\{j, M\}]]$

Also to leading order the function $A(x)$ will be simply given by

$$
\mathrm{A}[0]=\mathrm{F}[-\operatorname{Exp}[-\mathrm{L} * \operatorname{Cosh}[\mathrm{Pi} * \mathrm{x}]] * \operatorname{Product}[\mathrm{SO}[\mathrm{x}-\backslash[\mathrm{Theta}][0][[j]]+\mathrm{I} / 2] \sim 2,\{\mathrm{j}, \mathrm{M}\}]]
$$

Then we introduce the density $\rho$ and its conjugate $\bar{\rho}$ at the $k$-th iteration step as

$$
\begin{aligned}
& r\left[\mathrm{k}_{-}, \mathrm{y}_{-}\right]:=\log [(\mathrm{A}[\mathrm{k}][\mathrm{y}]-1) /(\operatorname{Abs}[\mathrm{A}[\mathrm{k}][\mathrm{y}]]-1)] ; \\
& \mathrm{rc}\left[\mathrm{k}_{-}, \mathrm{y}_{-}\right]:=\text {Conjugate }[\mathrm{r}[\mathrm{k}, \mathrm{y}]] ;
\end{aligned}
$$

Finally the code

$$
\begin{aligned}
& \mathrm{A}\left[\mathrm{k}_{-}\right]:=\mathrm{A}[\mathrm{k}]=\mathrm{F}[-\operatorname{Exp}[-\mathrm{L} * \operatorname{Cosh}[\mathrm{Pi} * \mathrm{x}]] \operatorname{Product}[\mathrm{S} 0[\mathrm{x}-\backslash[\text { Theta }][\mathrm{k}-1][[\mathrm{j}]]+\mathrm{I} / 2] \sim 2,\{\mathrm{j}, \mathrm{M}\}] \\
& \text { Exp [NIntegrate }[\mathrm{K} 0[\mathrm{x}-\mathrm{y}] \mathrm{r}[\mathrm{k}-1, \mathrm{y}]-\mathrm{K} 0[\mathrm{x}-\mathrm{y}+\mathrm{I}] * \mathrm{rc}[\mathrm{k}-1, \mathrm{y}]+1,\{\mathrm{y},-\mathrm{X}, \mathrm{x}, \mathrm{X}\} \text {, } \\
& \text { Method->PrincipalValue] -2X-rc }[\mathrm{k}-1, \mathrm{x}]]] \text {; } \\
& \text { phase }\left[\mathrm{k}_{-}\right]\left[\mathrm{x}_{-}\right]:=\mathrm{NIntegrate}[2 \operatorname{Im}[\mathrm{KO}[\mathrm{x}-\mathrm{y}-\mathrm{I} / 2] \mathrm{r}[\mathrm{k}-1, \mathrm{y}]]+1,\{\mathrm{y},-\mathrm{X}, \mathrm{X}\}]-2 \mathrm{X} \text {; } \\
& \backslash[\text { Theta }]\left[k_{-}\right]:=\backslash[\text { Theta }][k]=B A E[T a b l e[p h a s e[k][\backslash[\text { Theta }][k-1][[j]]],\{j, M\}]] \text {; }
\end{aligned}
$$

yields the $k$-th iteration quantities in terms of those computed at the $(k-1)$-th step. The energy 


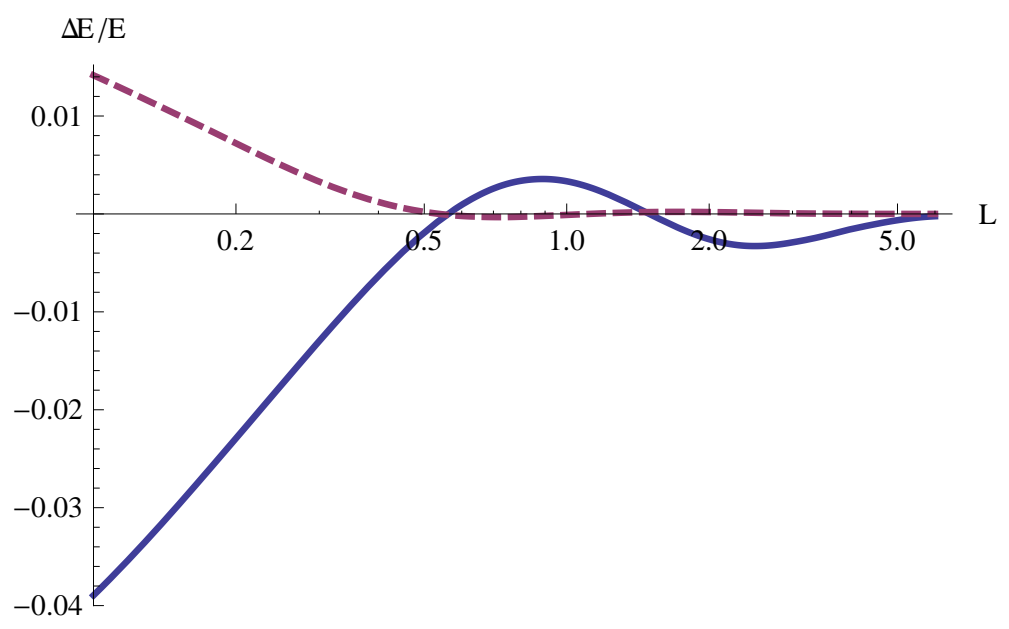

Figure 7: Difference between the predictions from the asymptotic Bethe ansatz and from the generalized Lüscher formulas to the exact (numerical) results for the two particle state polarized in both $S U(2)\left[u, v, \theta_{-11}\right]$. The solid (blue) line represents $\frac{E_{\text {exact }}-E_{A B A}}{E_{\text {exact }}}$ while the dashed (red) curve depicts $\frac{E_{\text {exact }}-E_{\text {Luscher }}}{E_{\text {exact }}}$. It is clear that the latter approximates the exact results with excellent accuracy, especially for large $L$ of course.

of the state is then given by

$$
\operatorname{En}\left[k_{-}\right]:=\operatorname{Sum}[\operatorname{Cosh}[\mathrm{Pi} \backslash[\operatorname{Theta}][\mathrm{k}][[j]]],\{j, \mathrm{M}\}]-\operatorname{NIntegrate}[\operatorname{Re}[\mathrm{r}[\mathrm{k}, \mathrm{y}]] \operatorname{Cosh}[\mathrm{Pi} * \mathrm{y}],\{\mathrm{y},-\mathrm{X}, \mathrm{X}\}] ;
$$

For example, to obtain the result of the first 8 iterations we simply run

$$
\text { Table }[\operatorname{En}[k],\{k, 0,8\}]
$$

to get $\{10.2414,10.2425,10.2425,10.2424,10.2424,10.2424,10.2424,10.2424\}$ where we notice that the iterations are clearly converging to the exact value 10.2424 up to the precision we are working at. It is instructive to compare these results to the value predicted by the asymptotic Bethe equations alone,

$$
E_{\mathrm{BAE}}=10.3388
$$

and to that predicted by the generalized Luscher formulae discussed in section 3.2 which gives

$$
E_{\text {Lüscher }}=10.2396
$$

\subsection{Discussion of numerical results}

Now we will try to interpret the behavior of various states on the fig.1 as functions of the volume $L$. Let us start from the vacuum, the lowest plot there. At very small $L$, the $O(4)$ model should become a 2d CFT of three massless bosons: if we introduce in (112) a rescaled field $\vec{Y}=e_{0}^{-1}(L)\left(X_{1}, X_{2}, X_{3}\right)$ and $X_{4}=\sqrt{1-e_{0}^{2}(L)\left(Y_{1}^{2}+Y_{2}^{2}+Y_{3}^{2}\right)}$, where $e_{0}^{2}(L) \simeq \frac{2 \pi}{|\log L|}$ is the effective charge, very small in this limit (the effective radius of the $S^{3}$ sphere $R(L)=e_{0}^{-1}(L)$ is very big), the action will be 


$$
\mathcal{S}_{\sigma}=\int d t d x \sum_{a=1}^{3}\left(\partial_{\alpha} Y_{a}\right)^{2}+O\left(e_{0}^{4}(L)\right) .
$$

In the ground state, the Casimir effect will define the limiting energy: $E_{0} \simeq-\frac{\pi c}{6 L}+O\left(1 / \log ^{4}\left(L^{-1}\right)\right)$, with the central charge $c=3$, which gives $E_{0} \frac{L}{2 \pi} \simeq-\frac{1}{4}$, the value compatible with -0.18 of the fig.1. 12

The energies of excited states are

$$
\frac{L}{2 \pi} E_{\vec{n}_{1} \vec{n}_{2} \vec{n}_{3} \ldots}(L) \simeq-\frac{1}{4}+\sum_{k=1}^{N} \sum_{\alpha=1}^{3}\left|n_{k}^{(\alpha)}\right|
$$

where $\vec{n}_{k}=\left(n_{k}^{(1)}, n_{k}^{(2)}, n_{k}^{(3)}\right)$ are the momentum numbers of particles constituting the state. We see that the small $L$ asymptotics of our plots are well described by this formula: The excited states in the $U(1)$ sector, denoted in the fig. 2 by $\theta_{n_{1}, n_{2} n_{3}, \cdots}$, according to the mode numbers $n_{1}, n_{2}, n_{3}$, approach the values predicted by (113) (up to the circumstance described in the last footnote). In this sector they have no excited left and right magnons (no $u, v$ roots), and only one component is activated: $\frac{L}{2 \pi} E_{n_{1} n_{2} n_{3} \ldots}(L)=\sum_{k}^{N} n_{k}^{(1)}$. Say, the curves $\theta_{0}, \theta_{00}, \theta_{000}, \theta_{0000}$, approach $-1 / 4$ at $L \rightarrow 0$, the curves $\theta_{1}, \theta_{01}, \theta_{001}$ approach $3 / 4$, the curve $\theta_{2}$ approaches $7 / 4$, etc. The state with one left and one right magnon excited, denoted as $\left[u, v, \theta_{-1,1}\right]$, also approaches $7 / 4$.

The qualitative behavior of the states $\theta_{0}, \theta_{00}, \theta_{000}, \theta_{0000}$,etc, at very small $L$ 's can be explained by the fact that the quantum fields are dominated by their zero modes. ${ }^{13}$ Since the momentum modes are not excited the field $\vec{Y}(\sigma, \tau)$ does not depend on $\sigma$. The action and the hamiltonian become:

$$
\mathcal{S}_{\sigma} \approx \frac{L}{e_{0}^{2}(L)} \int d t\left(\partial_{\tau} \phi\right)^{2}, \quad \hat{H}=\frac{1}{4} \frac{e_{0}^{2}(L)}{L} \hat{J}^{2}
$$

where the angle $\phi(\tau)$ represents the coordinate of a material point (a top) on the main circle of the unit sphere, and $\hat{J}$ is the corresponding angular momentum. The quantum mechanical spectrum of this system is well known:

$$
\frac{L}{2 \pi}\left(E_{\theta \text { times }}^{\{0,0, \ldots, 0\}}-E_{0}\right)=\frac{1}{8 \pi} e_{0}^{2}(L) m(m+2) \approx \frac{m(m+2)}{4 \log (\omega / L)}
$$

where $\omega=\gamma+\log \left(\frac{\sqrt{e}}{\sqrt{8}} \frac{\Gamma(3 / 2)}{4 \pi}\right) \simeq 13.66$ is a constant [46]. This formula explains well the fact that the corresponding plots on fig. 2 converge slowly, as inverse logarithm, to $-1 / 4$ and their spacing is approximately linearly growing with the number $m$.

The perturbative calculation of the mass gap $\left[E_{\theta_{0}}(L)-E_{0}(L)\right]$ for $L \ll 1$ was done in [46] and was compared with the numerical results following from the TBA approach in [26] . Since our numerics is in a perfect agreement with [26], for the states for which their method works, we will not review it here. We only recall that, in the logarithmic approximation,

\footnotetext{
${ }^{12}$ The convergence to the limiting value is very slow at $L \rightarrow 0$. At $L=0.1$ for our calculations we are still far from the limiting value of the energy. In [26], where the numerics reached $L=10^{-6}$, the result is -0.226 , considerably closer to the limiting value.

${ }^{13}$ We would like to thank A.Tsvelik, P.Wiegmann and K.Zarembo for the explanations on this subject.
} 


$$
\frac{L}{2 \pi}\left[E_{\theta_{0}}(L)-E_{0}(L)\right] \approx \frac{3}{4} \frac{1}{|\log L|}, \quad(L \ll 1)
$$

which is in the perfect agreement with eq.(115) at $m=1$. We also compared eq.(115) for $m=2$ with our numerics and found a good agreement ${ }^{14}$. The inverse logarithm in eq.(115) explains well the slight divergence of various curves with zero mode numbers at increasing $L$ in fig.2. Most probably, the divergence of the other plots at increasing $L$, corresponding to the same value of $\sum_{k=1}^{N} \sum_{\alpha=1}^{3}\left|n_{k}^{(\alpha)}\right|$ can be also perturbatively described by the same inverse log terms. It would be interesting to study the small $L$ limit analytically to recover analytic properties of the perturbation theory.

At large $L$, we enter the realm of the asymptotic Bethe ansatz with Lüscher-type exponentially small corrections to these regime. Actually, they describe very well our exact numerical plots for all the considered states, considerably beyond the values of $L$ allowed by the approximation, as seen in the plot 7 .

\section{Conclusions}

We derived in this paper the non-linear equations yielding the energy of an arbitrary excited state in the $O(4)$ two-dimensional sigma-model, equivalent to the $S U(2)$ principal chiral field, defined on a space circle of an arbitrary length $L$ (measured in infinite volume mass gap units). The main formulae we found are reviewed in subsection 6.1.

Although we considered mostly the $O(4)$ sigma model the new method which we develop here is very universal and should be applicable to any integrable relativistic sigma model, such as the $S U(N)$ principle chiral field at any $N, O(n)$ sigma models at any $n$, or more exotic models like SS-model or supersymmetric Sine-Gordon model (see [29] for the examples and the ground state energy and [11] for some excited states). In figure 8 the ground state Y-system diagrams for many known models are represented - it would be extremely interesting to perform a systematic study of such models using our formalism.

We also hope that the method will eventually allow to calculate the spectrum of finite size operators (such as the Konishi operator) in the N=4 SYM theory, when applied to its dual, the integrable string sigma model on $\mathrm{AdS}_{5} \times S^{5}$ background, on a world sheet cylinder of a finite circumference, as inspired by the works [38, 47, 48, 49]. In spite of the last spectacular applications of the S-matrix approach for the perturbative calculation of wrapping interactions for Konishi and other twist-2 operators [5], the problem of finding the dimensions of such operators at any coupling is still open.

The Hirota equation, which is equivalent to Y-system, appears to be a remarkable tool for solving the integrable sigma models in 2 space time dimensions. Not only does it help to collapse an infinite system of equations into a few ones, but it also helps to guess the analytic properties of the remaining unknown quantities and thus formulate the problem in terms of a single equation for a complex function $g(x)^{15}$. This function has the transparent meaning of a gauge transformation between the $T$ functions in two different solutions to the Hirota equation, but solving the same Y-system. This equation reminds of the famous Destri-deVega equation, and in the known cases,

\footnotetext{
${ }^{14}$ The discrepancy with the r.h.s. of eq.(115) for $m=2$ and $L=1 / 10,1 / 100,1 / 1000$ is $0.057,0.034,0.023$.

${ }^{15}$ It might be a few functions for other sigma models but always a finite number of them.
} 
like the chiral Gross-Neveu model, even coincides with it in certain variables, as we demonstrated in this paper. However, for many interesting sigma models the Destri-deVega equation is not known - in particular for general states in finite volume systems. Our method suggests a systematic way of deriving such DdV-like equations. For example, in the case of $S U(N)$ symmetry, we can expect that the closed set of such equations should not contain more than 4 real functions (or two complex)

- the total number of the gauge functions for the general Hirota equations. It would be interesting to apply our method to the $S U(N)$ principal chiral field [41, 42, 43], especially in the large $N$ limit, which is explicitly solvable for a non-zero magnetic field [50].

There could be interesting applications of our method to conformal QFT's in two dimensions if we consider them as some limiting cases of massive theories. These limiting cases could be the ultraviolet limit of a small volume $L \rightarrow \infty$ (see for example [14]) or analytic continuation w.r.t. the number of components of a field, like in [51], or something else.

Finally, one of the most promising grounds for the applications of our method should be the case of supersymmetric sigma models, a quickly developing subject, which is very useful in many physical problems ranging from AdS/CFT correspondence to disordered systems. The method of solution of Hirota equations applied for the supersymmetric spin chains with the symmetry algebras $g l(K \mid M)$, was worked out in $[52,53,39,54]$.

\subsection{Summary of the main formulae}

In this subsection we summarize our final integral equations in a self-consistent set of formulae. The main example considered in this paper was the $O(4)$ sigma model where the particles have two $S U(2)$ spins as internal degrees of freedom. To compute the exact energy of $N$-particle states with $J_{u}$ left spins down (and thus $N-J_{u}$ left spins up) and $J_{v}$ right spins down (and thus $N-J_{v}$ right spins up) we should solve the single integral equation on a complex function $g(x)$

$$
g(x)=i e^{\frac{i}{2} L \sinh (\pi x)} S(x) \exp \left[s * G_{v}(x-i / 2-i 0)-s * G_{u}(x+i / 2+i 0)\right],
$$

where $*$ stands for convolution, $s(x)=\frac{1}{2 \cosh \pi x}, S(x)=\prod_{j=1}^{N} S_{0}\left(x-\theta_{j}\right), S_{0}(x)=i \frac{\Gamma(1 / 2-i x / 2) \Gamma(+i x / 2)}{\Gamma(1 / 2+i x / 2) \Gamma(-i x / 2)}$ and the resolvents are given by

$$
G_{w}(x)=\int_{-\infty}^{+\infty} \frac{d y}{2 \pi i}\left(\frac{\rho_{w}(y)}{x-y-i / 2}-\frac{\bar{\rho}_{w}(y)}{x-y+i / 2}\right), \quad w=u, v
$$

where the densities are parameterized in terms of $g(x)$ as

$$
e^{\rho_{u}}=\frac{\frac{g^{+}}{\bar{g}^{+}} \mathcal{Q}_{u}^{++} \mathcal{Q}_{v}^{++}-\overline{\mathcal{Q}}_{u} \overline{\mathcal{Q}}_{v}}{\frac{g^{+}}{g^{-}} \mathcal{Q}_{u}^{++} \overline{\mathcal{Q}}_{v}^{--}-\mathcal{Q}_{u} \overline{\mathcal{Q}}_{v}}, e^{\rho_{v}}=\frac{\frac{g^{+}}{\bar{g}^{+}} \mathcal{Q}_{v}^{++} \mathcal{Q}_{u}^{++}-\overline{\mathcal{Q}}_{v} \overline{\mathcal{Q}}_{u}}{\bar{g}^{-} \mathcal{Q}_{v}^{++} \overline{\mathcal{Q}}_{u}^{--}-\mathcal{Q}_{v} \overline{\mathcal{Q}}_{u}},
$$

with $\mathcal{Q}_{w}(x)=\prod_{k=1}^{J_{w}}\left(x-w_{k}\right)$ and $\overline{\mathcal{Q}}_{w}(x)=\prod_{k=1}^{J_{w}}\left(x-\bar{w}_{k}\right)$ being the Baxter functions encoding the Bethe roots of the left and right "magnons" $(w=u, v)$. The superscripts \pm indicate the shifts by $\pm i / 2$, so that e.g. $\mathcal{Q}_{v}^{++}=\mathcal{Q}_{v}(x+i)$ and the bars indicate the complex conjugation. Finally the constants $\theta_{j}, u_{j}$ and $v_{j}$ are fixed by the finite volume Bethe equations

$$
\frac{\mathcal{Q}_{u}^{+}\left(\theta_{j}\right) \mathcal{Q}_{v}^{+}\left(\theta_{j}\right)}{\overline{\mathcal{Q}}_{u}^{-}\left(\theta_{j}\right) \overline{\mathcal{Q}}_{v}^{-}\left(\theta_{j}\right)} \frac{g\left(\theta_{j}\right)}{\bar{g}\left(\theta_{j}\right)}=1,-\frac{\phi^{-}\left(u_{j}\right) \mathcal{Q}_{u}^{++}\left(u_{j}\right)}{\phi^{+}\left(u_{j}\right) \overline{\mathcal{Q}}_{u}^{--}\left(u_{j}\right)} P_{u}\left(u_{j}\right)=1,-\frac{\phi^{-}\left(v_{j}\right) \mathcal{Q}_{v}^{++}\left(v_{j}\right)}{\phi^{+}\left(v_{j}\right) \overline{\mathcal{Q}}_{v}^{--}\left(v_{j}\right)} P_{v}\left(v_{j}\right)=1,
$$


where $P_{w=u, v}(x)$ is defined on the upper half plane by

$$
P_{w}(x)=\exp \left[K_{1}(x-i / 2) * \rho_{w}-K_{1}(x+i / 2) * \bar{\rho}_{w}\right], K_{1}(x)=\frac{2}{\pi} \frac{1}{4 x^{2}+1},
$$

and by its analytic continuation in the full complex plane. The energy of the state is then given by

$$
E=\sum_{k=1}^{N} \cosh \left(\pi \theta_{k}\right)-\frac{1}{2} \int_{-\infty}^{+\infty} \cosh (\pi x)\left(\rho_{u}(x)+\bar{\rho}_{u}(x)\right) d x .
$$

\section{Acknowledgements}

The work of NG was partly supported by the German Science Foundation (DFG) under the Collaborative Research Center (SFB) 676. The work of NG and VK was partly supported by the ANR grant INT-AdS/CFT (contract ANR36ADSCSTZ) and the grant RFFI 08-02-00287. The work of V.K. is also partly supported by the ANR grant GranMA BLAN-08-1-313695. N.G. and P.V. would like to thank Ecole Normale Superieure where a substantial part of this work was done. N.G. would also like to thank Commissariat ^ l'Energie Atomique where a substantial part of this work was done. V.K. would like to thank I.Kostov and Z.Tsuboi, P.Wiegmann for the useful discussions, and especially Anton Zabrodin, who shared with him his profound knowledge of discrete classical integrability. He also thanks the AEI (Potsdam) for the kind hospitality during the work on important parts of the project and the Humboldt fundation for the support. We also would like to thank A.Tsvelik, J.Teschner, V.Schomerus, K.Zarembo for many enlightening discussions, as well as A.Kozak who participated in this project on its the early stage.

\section{A Derivation of the (ground state) $Y$-system}

To compute the ground state energy $E_{0}(L)$ of the $S U(2)$ principal chiral field in a periodic box of a size $\mathrm{L}$ we can compute its Euclidean path integral $Z$ with the fields living on the space-time torus of the size $L \times R$, where the periodic imaginary "time" $R$ is very big

$$
Z=e^{-R E_{0}(L)} .
$$

Following Al.Zamolodchikov [17] we can compute this quantity exchanging the role of $L$ and $R$ so that the latter becomes the space variable whereas the former becomes the periodic time. Since $R \rightarrow \infty$ the spectrum corresponding to the new Hamiltonian can be computed from the asymptotic Bethe ansatz and the finite periodic time $L$ means that we should consider the system at a finite inverse temperature $L$. Thus we conclude that

$$
E_{0}(L)=f(L)
$$

where $f(L)$ is the free energy per unit length of the $S U(2) \mathrm{PCF}$ at the temperature $1 / L$ in the thermodynamical limit, when $R \rightarrow \infty$.

To compute the free energy we will start by reviewing the asymptotic spectrum of the theory as given by the asymptotic Bethe ansatz. Then we will recall what are the magnon bound states (complexes, or strings) and how they are organized in the complex plane. We will see that the quantum states in the thermodynamic limit can be described by the densities of these complexes 
and their holes. From this description we will write the entropy formula and thus find the desired exact free energy as the result of the saddle point approximation at $R \rightarrow \infty$. This will give the TBA equations.

Particles in the $S U(2)$ principal chiral field transform in the bi-fundamental representation under two $S U(2)$ groups. The theory is integrable and thus the general scattering process factorizes into a sequence of two-body scattering events. The $S$-matrix [33] describing the scattering of two particles with momenta and energies given by

$$
p_{j}=m \sinh \left(\pi \theta_{j}\right), E_{j}=m \cosh \left(\pi \theta_{j}\right),
$$

depends only on the difference of rapidities $\theta=\theta_{1}-\theta_{2}$

$$
\hat{S}_{12}(\theta)=S_{0}(\theta) \frac{\hat{R}(\theta)}{\theta-i} \otimes S_{0}(\theta) \frac{\hat{R}(\theta)}{\theta-i}, S_{0}(\theta)=i \frac{\Gamma\left(\frac{1}{2}-\frac{i \theta}{2}\right) \Gamma\left(+\frac{i \theta}{2}\right)}{\Gamma\left(\frac{1}{2}+\frac{i \theta}{2}\right) \Gamma\left(-\frac{i \theta}{2}\right)},
$$

where $\hat{R}(\theta)$ is the usual $S U(2)$ R-matrix in the fundamental representation given by

$$
\hat{R}(\theta)=\theta+i P,
$$

where $P$ is the permutation operator exchanging the spins of the scattered particles.

From now on, we will measure the length $L$ in the units of the mass gap $m$, which means that we will put $m=1$.

When $N$ particles are put on a large circle of length $R$ the periodicity condition to be imposed on the wave function reads

$$
-\hat{\mathcal{T}}\left(\theta_{j}\right) e^{i R \sinh \left(\pi \theta_{j}\right)} \Psi=\Psi,
$$

where $\mathcal{T}$ is the transfer matrix

$$
\left.\hat{\mathcal{T}}(\theta) \equiv \operatorname{tr}_{0}\left(\hat{S}_{01}\left(\theta-\theta_{1}\right) \ldots \hat{S}_{0 N}\left(\theta-\theta_{N}\right)\right)\right),
$$

with the index 0 for an additional auxiliary particle which we scatter against all physical particles. The trace is taken over this auxiliary space. Indeed, when the transfer matrix is evaluated at a value of the physical rapidity $\theta_{j}$ the corresponding $S$-matrix $\hat{S}_{0 j}\left(\theta-\theta_{j}\right)$ becomes simply $-P_{0 j} \otimes P_{0 j}$ which means that the auxiliary particle changes the quantum numbers and becomes the physical particle $\theta_{j}$. Then (122) becomes the periodicity condition (5) which physically states that once we pick the particle $j$ and carry it around the circle the total phase acquired by the wave function - which will be given by the free propagation $R p_{j}$ plus the phase shifts do to the (factorized) scattering with each of the other particles - must be a trivial multiple of $2 \pi$.

Using the algebraic or analytic Bethe ansatz technologies it is possible to diagonalize $\mathcal{T}(\theta)$ for any value of $\theta$ using the same eigenvector basis (see an Appendix from [55] for the details). Multiparticle states with $J_{u}$ left spins down (and thus $N-J_{u}$ left spins up) and $J_{v}$ right spins down (and thus $N-J_{v}$ right spins up) are parameterized by $J_{u}$ auxiliary Bethe roots $u_{j}$ and $J_{v}$ roots $v_{j}$ and

$$
\hat{\mathcal{T}}(\theta) \Psi=\frac{S^{2}(\theta)}{\phi^{2}(\theta-i)} T_{1}^{u}(\theta-i / 2) T_{1}^{v}(\theta-i / 2) \Psi
$$

where $T_{1}^{u(v)}$ is the transfer matrix in the fundamental representation associated with the left (right) $S U(2)$ spins,

$$
T_{1}^{w}(\theta) \equiv \frac{\mathcal{Q}_{w}(\theta+i) \phi(\theta-i / 2)+\mathcal{Q}_{w}(\theta-i) \phi(\theta+i / 2)}{\mathcal{Q}_{w}(\theta)},
$$


and

$$
\phi(\theta) \equiv \prod_{j=1}^{N}\left(\theta-\theta_{j}\right), \quad S(\theta)=\prod_{j=1}^{N} S_{0}\left(\theta-\theta_{j}\right), \quad \mathcal{Q}_{u}(\theta)=\prod_{j=1}^{J_{u}}\left(\theta-u_{j}\right), \quad \mathcal{Q}_{v}(\theta)=\prod_{j=1}^{J_{v}}\left(\theta-v_{j}\right) .
$$

The rapidities $\theta_{j}$ and $u_{j}, v_{j}$ are then fixed by a set of nested Bethe equations. The Bethe equations for the physical rapidities $\theta_{j}$ are given by the periodicity condition (122) which can be written as

$$
e^{-i m R \sinh \left(\pi \theta_{j}\right)}=-\frac{S^{2}\left(\theta_{j}\right)}{\phi^{2}\left(\theta_{j}-i\right)} T_{1}^{u}\left(\theta_{j}-i / 2\right) T_{1}^{v}\left(\theta_{j}-i / 2\right),
$$

or simply

$$
e^{-i m R \sinh \left(\pi \theta_{j}\right)}=-S^{2}\left(\theta_{j}\right) \frac{\mathcal{Q}_{u}\left(\theta_{j}+i / 2\right)}{\mathcal{Q}_{u}\left(\theta_{j}-i / 2\right)} \frac{\mathcal{Q}_{v}\left(\theta_{j}+i / 2\right)}{\mathcal{Q}_{v}\left(\theta_{j}-i / 2\right)} .
$$

The magnon rapidities $u_{j}$ and $v_{j}$ are fixed by the auxiliary Bethe equations

$$
-\frac{\mathcal{Q}_{u}\left(u_{j}+i\right)}{\mathcal{Q}_{u}\left(u_{j}-i\right)}=\frac{\phi\left(u_{j}+i / 2\right)}{\phi\left(u_{j}-i / 2\right)},-\frac{\mathcal{Q}_{v}\left(v_{j}+i\right)}{\mathcal{Q}_{v}\left(v_{j}-i\right)}=\frac{\phi\left(v_{j}+i / 2\right)}{\phi\left(v_{j}-i / 2\right)},
$$

which appear in the diagonalization of the left and right transfer matrices. Notice that these equations ensure that the apparent poles in (125) drop out and render $T_{1}^{w}(\theta)$ polynomial as it ought to be. For each solution to these equations we obtain the energy of the corresponding quantum state from

$$
E=\sum_{j=1}^{N} \cosh \left(\pi \theta_{j}\right)
$$

To be able to compute the free energy $f(L)$ we need to understand how the solutions to these Bethe equations organize themselves so that we can introduce the entropy density. Let us consider the auxiliary roots $u$, obviously the same considerations will apply for the $v$ roots. These roots can take complex values. When $u_{j}$ has a positive imaginary part the r.h.s of the Bethe equations in (129) diverges,

$$
\frac{\phi\left(u_{j}+i / 2\right)}{\phi\left(u_{j}-i / 2\right)} \stackrel{N \rightarrow \infty}{\rightarrow} \infty
$$

which means that

$$
\frac{\mathcal{Q}_{u}\left(u_{j}+i\right)}{\mathcal{Q}_{u}\left(u_{j}-i\right)}
$$

must diverge as well. This can be achieved if there is another magnon rapidity $u_{k}$ such that $u_{j}-u_{k} \simeq i$. Thus, in the thermodynamical limit the magnon rapidities will organize themselves into a Bethe-string of $n$ roots $u_{j}$ spaced by $i$. In particular, a single real root corresponds to a Bethe string with $n=1$. The Bethe equations can then be multiplied for $u_{j}$ 's belonging to the same string so that this gives new Bethe equations, solely for the (real) center of each string. This is the usual fusion procedure applied at the level of the Bethe equations. The resulting equations look as follows. Introduce the magnon bound states:

$$
u_{j, a}^{(n)}=u_{j}^{(n)}+i \frac{1}{2}(n+1)-i a, \quad a=1, \ldots, n .
$$


Multiplying the equations for a given $n$-bound state we get for eq.(129) and eq.(128)

$$
\begin{aligned}
e^{-i R p\left(\theta_{\alpha}\right)} & =\prod_{\beta \neq \alpha} S_{0}^{2}\left(\theta_{\alpha}-\theta_{\beta}\right) \prod_{j, n} \frac{\theta_{\alpha}-u_{j}^{(n)}+i \frac{n}{2}}{\theta_{\alpha}-u_{j}^{(n)}-i \frac{n}{2}}, \\
\prod_{\beta} \frac{u_{j}^{(n)}-\theta_{\beta}+i \frac{n}{2}}{u_{j}^{(n)}-\theta_{\beta}-i \frac{n}{2}} & =\prod_{(k, m) \neq(j, n)} \frac{u_{j}^{(n)}-u_{k}^{(m)}-i \frac{n+m}{2}}{u_{j}^{(n)}-u_{k}^{(m)}+i \frac{n+m}{2}} \cdot \frac{u_{j}^{(n)}-u_{k}^{(m)}-i \frac{|n-m|}{2}}{u_{j}^{(n)}-u_{k}^{(m)}+i \frac{|n-m|}{2}} \prod_{s=\frac{|n-m|}{2}}^{\frac{n+m}{2}}\left(\frac{u_{j}^{(n)}-u_{k}^{(m)}+i s}{u_{j}^{(n)}-u_{k}^{(m)}-i s}\right)^{2} .
\end{aligned}
$$

In the thermodynamic limit we will have a large number of each type of Bethe roots which we can describe by a density $\varrho_{n}$. We use $n=0$ for the density of $\theta$ particles, $n \geq 1$ to describe the density of $u$ Bethe strings of size $n$ and $n \leq-1$ for the $v$ Bethe strings made out of $-n$ roots. For each density of particles we also have the corresponding density of holes $\bar{\varrho}_{n}$. Bethe equations in the thermodynamic limit, obtained by taking the logarithmic derivatives of both sides of these equations, read

$$
\varrho_{n}+\bar{\varrho}_{n}=\frac{R}{2} \cosh (\pi \theta) \delta_{n 0}-\sum_{m=-\infty}^{\infty} K_{n, m} * \varrho_{m}
$$

where $*$ stands for the usual convolution

$$
f * g=\int_{-\infty}^{+\infty} d \theta^{\prime} f\left(\theta-\theta^{\prime}\right) g\left(\theta^{\prime}\right)
$$

and $K_{n m}$ is the derivative of the logarithm of the effective $S$-matrix between the strings of size $n$ and $m$. In particular we have

$$
-K_{0,0}(\theta) \equiv K_{0}(\theta)=\frac{1}{2 \pi i} \frac{d}{d \theta} \log S_{0}^{2}(\theta)
$$

for the interaction between physical rapidities,

$$
K_{0, n}(\theta)=-K_{n, 0}(\theta)=\frac{1}{2 \pi i} \frac{d}{d \theta} \log \frac{\theta-i|n| / 2}{\theta+i|n| / 2}=\frac{1}{\pi} \frac{2|n|}{4 \theta^{2}+|n|^{2}} \equiv K_{n}(\theta), \quad n \neq 0,
$$

for the interaction of $\theta$ rapidities with Bethe strings of size $|n|$ and

$$
K_{n, m}(\theta)=K_{-n,-m}(\theta)=\sum_{s=\frac{|n-m|}{2}+1}^{\frac{n+m}{2}} 2 K_{2 s}(\theta)-K_{n+m}(\theta)+K_{|n-m|}(\theta) \delta_{n \neq m}, n, m=1,2, \ldots
$$

for the interaction of two Bethe strings. Obviously $K_{n, m}=0$ if $n \times m<0$.

It is interesting that even though these kernels appear as some quite complicated functions they all exhibit very simple fourier transforms $\hat{K}_{n, m}$. More precisely we have

$$
\hat{K}_{0}(\omega)=\frac{e^{-|\omega| / 2}}{\cosh \frac{\omega}{2}}
$$

and

$$
\hat{K}_{n}(\omega)=e^{-|n| \omega / 2}, n=1,2, \ldots,
$$


so that the sum in (137) can be explicitly done yielding

$$
\hat{K}_{n, m}=\operatorname{coth}\left(\frac{|\omega|}{2}\right)\left(e^{-\frac{|\omega|}{2}|m-n|}-e^{-\frac{|\omega|}{2}(m+n)}\right)-\delta_{n, m}, n, m=1,2, \ldots
$$

A very useful formula for what follows concerns the inversion of the operator $K_{n m}$ when both indices $n$ and $m$ are restricted to be positive (or negative). In Fourier space

$$
\left(\hat{K}_{n m}+\delta_{n m}\right)^{-1}=\delta_{m n}-\hat{s}\left(\delta_{n, m+1}+\delta_{n, m-1}\right), \quad(n, m>0),
$$

where the operator $\hat{s}$ (and its fourier transform) has the following form

$$
s(\theta)=\frac{1}{2 \cosh \pi \theta}, \quad\left(\hat{s}(\omega)=\frac{1}{2 \cosh \left(\frac{\omega}{2}\right)}\right) .
$$

In particular we notice that

$$
K_{0}=2 s * K_{1} .
$$

Having introduced all the necessary kernels we can proceed to construct the quantity of interest, the free energy at the temperature $1 / L$. We have

$$
f(L)=\min _{\varrho_{n}, \bar{\varrho}_{n}} \int d \theta\left(\varrho_{0} L \cosh \pi \theta-\sum_{n=-\infty}^{\infty} \varrho_{n} \log \left(1+\frac{\bar{\varrho}_{n}}{\varrho_{n}}\right)+\bar{\varrho}_{n} \log \left(1+\frac{\varrho_{n}}{\bar{\varrho}_{n}}\right)\right),
$$

where we should minimize the integral by varying the densities of particles and holes keeping the Bethe equations satisfied. The first term in the integral is the energy density multiplied by the inverse temperature (which is $L$ ) and the second term given by the sum over $n$ is the entropy density (see e.g. [17]). We use Bethe equations to write the variation $\delta \varrho_{n}=-\delta \varrho_{n}-\sum_{m=-\infty}^{\infty} K_{n m} * \delta \varrho_{m}$ so that the extremum condition $\delta f=0$ yields a set of TBA equations

$$
0=-\epsilon_{n}+L \cosh (\pi \theta) \delta_{n, 0}+\sum_{m=-\infty}^{\infty} K_{m n} * \log \left(1+e^{-\epsilon_{m}}\right)
$$

where $\frac{\underline{\varrho}_{n}}{\bar{\varrho}_{n}}=e^{-\epsilon_{n}}$. The free energy evaluated at this extremum can then be written in terms of $\epsilon_{0}$ alone, so that

$$
E_{0}(L)=-\int \frac{d \theta}{2} \cosh (\pi \theta) \log \left(1+e^{-\epsilon_{0}(\theta)}\right) .
$$

The last two equations yield the finite size ground state energy of the $S U(2)$ principal chiral field.

Finally, we will show below that defining the incidence matrix $I_{n m}=\delta_{n, m \pm 1}$, the $Y$ variables $Y_{m}=e^{\epsilon_{m}}(m \neq 0), Y_{0}=e^{-\epsilon_{0}}$ and using the operator (141) these equations can be transformed into a local (in the discrete variable $n$ ) set of integral equations

$$
\log \left(Y_{n}\right)+L \cosh (\pi \theta) \delta_{n, 0}=\sum_{m=-\infty}^{\infty} I_{n m} s * \log \left(1+Y_{m}\right), \quad-\infty<n<\infty
$$

and

$$
E_{0}(L)=-\int \frac{d \theta}{2} \cosh (\pi \theta) \log \left(1+Y_{0}\right)
$$


It is remarkable that non-local kernels $K_{n m}$ disappear and at the end only a very simple kernel $\hat{s}$ appears in the final set of TBA equations. It is also remarkable, and still somewhat mysterious, that the $S U(2)_{L}$ and $S U(2)_{R}$ wings are smoothly glued into one Y-system on a discrete set $-\infty<n<\infty$.

To show (147) we should consider (145) separately for $n>0, n<0$ and $n=0$. Applying the operator (141) to (145) in the first two cases (it is convenient to rearrange them to have the combination $\delta_{m n}+K_{m n}$ ), we easily verify (147), except the case $n=0$ which we should consider separately. To find $n=0$ equation of the Y-system we consider (145) for $n=-1,0,1$. The kernels $K_{n, m}$ entering these three equations are

$$
-K_{0,0}=K_{0}=2 s * K_{1}, K_{0, \pm 1}=K_{1}, K_{ \pm m, 0}=-K_{m}, m>0
$$

and, most importantly,

$$
K_{ \pm m, \pm 1}=K_{m+1}+K_{m-1} \delta_{m \neq 1} .
$$

Thus if we convolute (145) for $n=1$ with the inverse shift operator $s$ and use that $s *\left(K_{m+1}+\right.$ $\left.K_{m-1}\right)=K_{m}$ we get

$$
0=-s * K_{1} * \log \left(1+e^{-\epsilon_{0}}\right)+\sum_{m=1}^{\infty} K_{m} * \log \left(1+e^{-\epsilon_{m}}\right)-s * \log \left(1+e^{+\epsilon_{1}}\right) .
$$

Notice that the last term is separated from the infinite sum because the $m=1$ case in (150) behaves slightly differently than for the other $m$ 's. Moreover the sign of the exponent inside this log differs from that inside the logs in the infinite sum because we absorbed the first term in (145) into this last log. Similarly, for $n=-1$ we have

$$
0=-s * K_{1} * \log \left(1+e^{-\epsilon_{0}}\right)+\sum_{m=-\infty}^{-1} K_{|m|} * \log \left(1+e^{-\epsilon_{m}}\right)-s * \log \left(1+e^{+\epsilon_{-1}}\right) .
$$

These two equations can then be used to simplify the $n=0$ equation which reads

$$
0=\epsilon_{0}-L \cosh (\pi \theta)+K_{0} * \log \left(1+e^{-\epsilon_{0}}\right)-\sum_{m \neq 0} K_{|m|} * \log \left(1+e^{-\epsilon_{m}}\right) .
$$

Indeed if we sum all these three equations we see that (i) the infinite sums completely cancel out, (ii) the convolutions with $\log \left(1+e^{-\epsilon_{0}}\right)$ drop out as well by virtue of the identity $K_{0}=2 s * K_{1}$. We are thus left with the last terms in (151) and (152) plus the first two terms in (153) thus obtaining the last $Y$-system equation (147) for $n=0$.

Moreover, for the functions $g(\theta)$ analytic inside the physical strip $\operatorname{Im}(\theta)<1 / 2$ we have

$$
s *[g(\theta+i / 2)+g(\theta-i / 2)]=g(\theta)
$$

because

$$
\int_{R} \frac{g(\theta+i / 2)+g(\theta-i / 2)}{2 \cosh (\pi(\theta-x))} d \theta=\frac{1}{2 i} \oint_{\mathrm{PS}} \frac{g(\theta)}{\sinh (\pi(\theta-x))} d \theta=g(x) .
$$

Therefore if $Y_{n}$ is non-zero inside the physical strip we can invert the $s$ operator to find a set of functional equations, finally rendering the $Y$-system for the $\mathrm{PCF}$ at a finite temperature $1 / L$

$$
Y_{n}(\theta+i / 2) Y_{n}(\theta-i / 2)=\left(1+Y_{n-1}(\theta)\right)\left(1+Y_{n+1}(\theta)\right)
$$

To fix a solution, this $Y$-system ought to be supplemented by the large $\theta$ asymptotics $Y_{n} \simeq$ $e^{-\delta_{n 0} L \cosh \pi \theta}$ related to the relativistic dispersion relation. Notice that these functional equations do not contain the dispersion relation explicitly: it appears only through the asymptotics of the $Y$-functions. 


\section{B General solution in terms of Hirota functions}

In this appendix, we will give an alternative approach to the construction of solution for the energy of a general state of the $S U(2) \mathrm{PCF}$ in the periodic box. It will give a new NLIE defining the spectrum, different from the one of the section 3. This approach is the generalization of the approach we used in section 2.7 for the states with $N$ particles and non-trivial polarizations encoded in the Baxter polynomials $\mathcal{Q}_{u}$ and $\mathcal{Q}_{v}$.

As explained in the beginning of the section 3, for each solution to the Y-system equations, there are two natural solutions to Hirota equation which yield the same $Y$ 's and are related by a gauge transformation. The expected analytic properties of these functions are described in this section. In particular we have

$$
\begin{aligned}
T_{+1}^{v} & =g^{+} \bar{g}^{-} T_{+1}^{u} \\
T_{-1}^{u} & =\frac{1}{g^{-} \bar{g}^{+}} T_{-1}^{v} \\
\Phi_{v} & =g^{+} g^{-} \Phi_{u}
\end{aligned}
$$

which we will now use to completely solve our problem. First, as in the section 3 , we find the gauge function $g$ from the last relation,

$$
g=i e^{i L \sinh (\pi x) / 2}\left(\frac{\Phi^{v}}{\Phi^{u}}\right)^{* s}, \quad \bar{g}=-i e^{-i L \sinh (\pi x) / 2}\left(\frac{\bar{\Phi}^{v}}{\bar{\Phi}^{u}}\right)^{* s} .
$$

and plug it in the first two to find

$$
\begin{aligned}
T_{+1}^{v} & =e^{-L \cosh (\pi x)} \frac{\Phi_{v} \bar{\Phi}_{v} T_{+1}^{u}}{\left(\Phi_{u}^{+} \Phi_{v}^{-} \bar{\Phi}_{v}^{+} \bar{\Phi}_{u}^{-}\right)^{* s}} \\
T_{-1}^{u} & =e^{-L \cosh (\pi x)} \frac{\Phi_{u} \bar{\Phi}_{u} T_{-1}^{v}}{\left(\Phi_{u}^{+} \Phi_{v}^{-} \bar{\Phi}_{v}^{+} \bar{\Phi}_{u}^{-}\right)^{* s}}
\end{aligned}
$$

As in the section 3 , we still have to relate $T_{0}^{u, v}$ and $\Phi^{u, v}$, but we do it here by a different relation. For that, let us define in the whole complex plane $x$ the functions

$$
F_{u}(x)=\phi(x)-\frac{\phi(x)}{2 \pi i} \int \frac{T_{-1}^{u}(y) \mathcal{Q}_{u}(y+i) / \mathcal{Q}_{u}(y)}{(y-x+i / 2) \phi(y+i / 2)} d y+\frac{\phi(x)}{2 \pi i} \int \frac{T_{-1}^{u}(y) \overline{\mathcal{Q}}_{u}(y-i) / \overline{\mathcal{Q}}_{u}(y)}{(y-x-i / 2) \phi(y-i / 2)} d y
$$

and

$$
F_{v}(x)=\phi(x)-\frac{\phi(x)}{2 \pi i} \int \frac{T_{+1}^{v}(y) \mathcal{Q}_{v}(y+i) / \mathcal{Q}_{v}(y)}{(y-x+i / 2) \phi(y+i / 2)} d y+\frac{\phi(x)}{2 \pi i} \int \frac{T_{+1}^{v}(y) \overline{\mathcal{Q}}_{v}(y-i) / \overline{\mathcal{Q}}_{v}(y)}{(y-x-i / 2) \phi(y-i / 2)} d y,
$$

where the integrals essentially go along the real axis, but we should pass the contour in such a way that the zeroes $u_{j}, v_{j}$ of $\mathcal{Q}_{u, v}$ remain below the contour and the complex conjugated zeroes $\bar{u}_{j}$ of $\overline{\mathcal{Q}}_{u, v}$ stay above the contour. Using eq.(22) we can show that $T_{0}^{u, v}$ and $\Phi_{u, v}$ are related to the values of the same analytic function $F_{u, v}$ inside and outside of the analyticity strip, respectively:

$$
F_{u}(x)=\left\{\begin{array}{cl}
\frac{\overline{\mathcal{Q}}_{u}^{-}}{\mathcal{Q}_{u}^{-}} \Phi_{u}^{-} & \operatorname{Im} x>+1 / 2 \\
T_{0}^{u}(x) & |\operatorname{Im} x|<1 / 2 \\
\frac{\mathcal{Q}_{u}^{+}}{\overline{\mathcal{Q}}_{u}^{+}} \bar{\Phi}_{u}^{+} & \operatorname{Im} x<-1 / 2
\end{array} \quad, \quad F_{v}(x)=\left\{\begin{array}{cl}
-\frac{\overline{\mathcal{Q}}_{v}^{-}}{\mathcal{Q}_{v}^{-}} \bar{\Phi}_{v}^{-} & \operatorname{Im} x>+1 / 2 \\
T_{0}^{v}(x) & |\operatorname{Im} x|<1 / 2 \\
-\frac{\mathcal{Q}_{v}^{+}}{\overline{\mathcal{Q}}_{v}^{+}} \Phi_{v}^{+} & \operatorname{Im} x<-1 / 2
\end{array}\right.\right.
$$


Indeed, substituting from $(22) T_{-1}^{u}=\left(T_{0}^{u+} \mathcal{Q}^{u}-\Phi^{u} \overline{\mathcal{Q}}^{u}\right) / \mathcal{Q}^{u++}$ and the conjugate $T_{-1}^{u}=\left(T_{0}^{u-} \overline{\mathcal{Q}}^{u}-\right.$ $\left.\bar{\Phi}^{u} \mathcal{Q}^{u}\right) / \bar{Q}^{u--}$ into the first and second terms of (159), respectively, we can separate the contribution of two integrals in (159) containing $T_{0}^{u}$ into a single contour integral going around the physical strip (to realize it is useful to make shifts by $\pm i / 2$ for the integration variable). We shall also use the fact that $\frac{T_{0}^{u}}{\phi^{+}} \rightarrow 1$ at $x \rightarrow \pm \infty$. If the point $x$ is inside the physical strip we can contract the contour around the pole at $y=x$ and thus verify the middle relation in eq.(161). The other two integrals in (161), containing $\Phi$ and $\bar{\Phi}$, do not contribute since we can close the contours there around the upper and lower half-plane, respectively. The result is zero since there are no singularities inside by our previous assumption. The poles related to zeroes of $\mathcal{Q}_{u, v}$ do not contribute since they are outside of these contours by definition. We should ensure by hand the analyticity of $\Phi(x)$ close to the real axis

$$
T_{0}^{u+} \mathcal{Q}^{u}=T_{-1}^{u} \mathcal{Q}^{u++}, x=\bar{u}_{j},
$$

which is the finite $L$ deformation of the usual asymptotic axillary BAE for the $u$-roots. The relations for $F_{v}$ are found in a similar way. This equation can be shown to be equivalent to the eq.(70) derived in the main text.

Notice that $T_{0}^{u}$ and $T_{0}^{v}$ are automatically analytic even slightly outside of the physical strip, because we can deform the contours to open further the physical strip. We will discuss this "extra" analyticity in the App.D.

Finally, notice also that

$$
\begin{aligned}
T_{1}^{u}(x) & =\frac{\mathcal{Q}_{u}(x+i)}{\mathcal{Q}_{u}(x)} F_{u}(x-i / 2)+\frac{\overline{\mathcal{Q}}_{u}(x-i)}{\overline{\mathcal{Q}}_{u}(x)} F_{u}(x+i / 2), \\
T_{-1}^{v}(x) & =\frac{\mathcal{Q}_{v}(x+i)}{\mathcal{Q}_{v}(x)} F_{v}(x-i / 2)+\frac{\overline{\mathcal{Q}}_{v}(x-i)}{\overline{\mathcal{Q}}_{v}(x)} F_{v}(x+i / 2),
\end{aligned}
$$

so that (158) completely constrain the functions $T_{-1}^{u}$ and $T_{1}^{v}$ out of which all other $T_{k}$ and $Y_{k}$ can be written down using the resolvents $F_{u}$ and $F_{v}$.

In the $U(1)$ sector we have $\mathcal{Q}_{u}=\mathcal{Q}_{v}=1$ and the two wings are obviously equivalent. We will have in this case $T_{1}^{v}=T_{-1}^{u}$ and $F_{u}=F_{v}$ will be given by (47). Equations (158) then reduce to the previously derived equation (51).

\section{B.1 The main Bethe equations}

The main BAE reflecting the periodicity of the wave function and constraining the real zeroes $\theta_{j}$ is given by (16):

$$
Y_{0}\left(\theta_{j} \pm i / 2\right)=\frac{T_{1}^{u}\left(\theta_{j} \pm i / 2\right) T_{-1}^{u}\left(\theta_{j} \pm i / 2\right)}{\Phi_{u}\left(\theta_{j} \pm i / 2\right) \bar{\Phi}_{u}\left(\theta_{j} \pm i / 2\right)}=-1 .
$$

Using the fact that $T_{1}^{u}$ has no zeros inside the physical strip and the denominator is regular for real $\theta_{j}$ we conclude that $T_{-1}^{u}\left(\theta_{j} \pm i / 2\right) \neq 0$. This condition can be in fact interpreted as yet another form of the main BAE. It can be further simplified: using (156) we get

$$
T_{-1}^{u+}=\frac{1}{g \bar{g}^{++}} T_{-1}^{v+}=-\frac{\bar{g} \bar{\Phi}_{u}^{+}}{g \bar{\Phi}_{v}^{+}} T_{-1}^{v+}
$$

where we can use that, due to (22), for the $u$-wing

$$
\bar{\Phi}_{u}^{+}=+T_{0}^{u} \frac{\overline{\mathcal{Q}}_{u}^{+}}{\mathcal{Q}_{u}^{+}}-T_{-1}^{u+} \frac{\overline{\mathcal{Q}}_{u}^{-}}{\mathcal{Q}_{u}^{+}} .
$$


Substituting it into the eq.(164), and evaluating at $\theta_{j}$ which is a zero of $T_{0}$ we get

$$
1=\left.\frac{\bar{g}}{g} \frac{\overline{\mathcal{Q}}_{u}^{-}}{\mathcal{Q}_{u}^{+}} \frac{T_{-1}^{v+}}{\bar{\Phi}_{v}^{+}}\right|_{x=\theta_{j}},
$$

or, using (22) for the $v$-wing, we get the simplest form of the main equation, easy to compare with the large $L$ limit

$$
1=\left.\frac{\bar{g}}{g} \frac{\overline{\mathcal{Q}}_{u}^{-}}{\mathcal{Q}_{u}^{+}} \frac{\overline{\mathcal{Q}}_{v}^{-}}{\mathcal{Q}_{v}^{+}}\right|_{x=\theta_{j}} .
$$

which was also derived in the main text eq.(67).

\section{Proof of reality of $T_{k}$}

In this appendix we shall analyze the reality of the $T$-functions. This is an important point to consider because the Hirota equation is solved explicitly by eq.(23) provided all $T_{k}$ are real. The goal of this appendix is to show that once the following equations (see (58)) are satisfied

$$
\begin{aligned}
\frac{T_{0}^{u+}}{\Phi_{u}} & =\frac{\frac{g^{+}}{\bar{g}^{+}} \mathcal{Q}_{u}^{++} \mathcal{Q}_{v}^{++}-\overline{\mathcal{Q}}_{u} \overline{\mathcal{Q}}_{v}}{\frac{g^{+}}{g^{-}} \mathcal{Q}_{u}^{++} \overline{\mathcal{Q}}_{v}^{--}-\mathcal{Q}_{u} \overline{\mathcal{Q}}_{v}}, \\
-\frac{T_{0}^{v+}}{\bar{\Phi}_{v}} & =\frac{\frac{g^{+}}{\bar{g}^{+}} \mathcal{Q}_{v}^{++} \mathcal{Q}_{u}^{++}-\overline{\mathcal{Q}}_{v} \overline{\mathcal{Q}}_{u}}{\overline{\bar{g}}^{-} \mathcal{Q}_{v}^{++} \overline{\mathcal{Q}}_{u}^{--}-\mathcal{Q}_{v} \overline{\mathcal{Q}}_{u}} \\
\bar{g}_{v} & =g^{+} g^{-} \Phi_{u},
\end{aligned}
$$

and $T_{0}^{u}$ and $T_{0}^{v}$ are real then all $T_{k}$ are real and thus all the formulae in the main text go through and the $Y$-system is indeed solved by (18). Before proving this statement we recall that eq.(66) follows directly from eq.(170) under certain analyticity assumptions and also from $\frac{T_{0}^{u+} T_{0}^{u-}}{\Phi_{u} \Phi_{u}}=\frac{T_{0}^{v+} T_{0}^{v-}}{\Phi_{v} \Phi_{v}}$, which is a consequence of eq.(168) and eq.(169). Thus we can add the equation

$$
T_{0}^{v}=g \bar{g} T_{0}^{u}
$$

to the equations at hand and proceed to the proof of the reality of the $T$-functions.

Equation (168) implies

$$
\Phi_{u}=i h_{1}^{+}\left(\frac{\mathcal{Q}_{u}^{++} \overline{\mathcal{Q}}_{v}^{--}}{g^{-}}-\frac{\mathcal{Q}_{u} \overline{\mathcal{Q}}_{v}}{g^{+}}\right), T_{0}^{u}=i h_{1}\left(\frac{\mathcal{Q}_{u}^{+} \mathcal{Q}_{v}^{+}}{\bar{g}}-\frac{\overline{\mathcal{Q}}_{u}^{-} \overline{\mathcal{Q}}_{v}^{-}}{g}\right),
$$

for some $h_{1}$. Since $T_{0}^{u}$ is real for real $x$ the function $h_{1}$ is a real function. Eq.(169) implies

$$
\bar{\Phi}_{v}=-i h_{2}^{+}\left(\bar{g}^{-} \mathcal{Q}_{v}^{++} \overline{\mathcal{Q}}_{u}^{--}-\bar{g}^{+} \mathcal{Q}_{v} \overline{\mathcal{Q}}_{u}\right), T_{0}^{v}=i h_{2}\left(g \mathcal{Q}_{u}^{+} \mathcal{Q}_{v}^{+}-\bar{g} \overline{\mathcal{Q}}_{u}^{-} \overline{\mathcal{Q}}_{v}^{-}\right),
$$

where again $h_{2}$ is a real function. In virtue of (171) we have $h_{1}=h_{2} \equiv h$ and by conjugating the first equation in (173) we find

$$
\Phi_{v}=i h^{-}\left(g^{+} \overline{\mathcal{Q}}_{v}^{--} \mathcal{Q}_{u}^{++}-g^{-} \overline{\mathcal{Q}}_{v} \mathcal{Q}_{u}\right)=\frac{h^{-}}{h^{+}} g^{-} g^{+} \Phi_{u},
$$


which means that the real function $h(x)$ is periodic in the imaginary direction, $h(x)=h(x+i)$. This in turn implies that the function $h^{-}$is also a real function because

$$
\overline{h(x-i / 2)}=h(x+i / 2)=h(x-i / 2) \text {. }
$$

Then it is simple to see that $T_{1}^{u}$ is real. We simply write, from eq.(172),

$$
T_{1}^{u}=T_{0}^{u-} \frac{\mathcal{Q}_{u}^{++}}{\mathcal{Q}_{u}}+\Phi_{u} \frac{\overline{\mathcal{Q}}_{u}^{--}}{\mathcal{Q}_{u}}=i h^{-}\left(\frac{\mathcal{Q}_{v} \mathcal{Q}_{u}^{++}}{\bar{g}^{-}}-\frac{\overline{\mathcal{Q}}_{v} \overline{\mathcal{Q}}_{u}^{--}}{g^{+}}\right)
$$

The reality of $T_{1}^{u}$ is now manifest because the expression inside the brackets is purely imaginary and, as we have just shown, $h^{-}$is real. Proceeding in the same way one can see that all $T_{k}^{u}$ are real and thus the Hirota equation is satisfied by our solution.

\section{Proof of analyticity of $T_{-1}$ in the physical strip}

\section{D.1 Analyticity in the $U(1)$ sector}

From (51) together with $F(x \pm i / 2 \pm i 0)=F(x \pm i / 2 \mp i 0)-T_{-1}(x)$ we have

$$
T_{-1}(x)=\frac{T_{1} F^{++} F^{-+}}{\left[F^{++} F^{--}\right]^{* 2 s} e^{L \cosh (\pi x)}+T_{1} F^{++}}=\frac{T_{1} F^{+-} F^{--}}{\left[F^{++} F^{--}\right]^{* 2 s} e^{L \cosh (\pi x)}+T_{1} F^{--}} .
$$

Using respectively the first/second equality we can smoothly move from real $x$ into the upper/lower half complex plane provided $T_{1}$ is analytic. In this way we can reach any $x$ inside the enlarged strip $|\operatorname{Im}(x)|<1$ where $T_{1}$ is regular.

Notice that for large $L$ we have

$$
T_{-1}(x) \simeq \begin{cases}\phi^{-} & ,+1 / 2<\operatorname{Im}(x)<+1 \\ 0 & ,-1 / 2<\operatorname{Im}(x)<+1 / 2 \\ \phi^{+} & ,-1<\operatorname{Im}(x)<-1 / 2\end{cases}
$$

The denominator in the expression for $T_{-1}$ at $x=\theta_{j}-i / 2$ is proportional to Bethe equations

$S^{2} e^{i p_{j} L}+1=0$. This is not a pole of $T_{-1}$ because the numerator at these points is proportional to $T_{0}\left(\theta_{j}\right)=0$.

However, for large volume, $T_{-1}$ could have poles at the analogue of the holes of the $\theta_{j} \mathrm{BAE}$, close to the boundaries of the physical strip.

\section{D.2 General case}

In this subsection we will study the analyticity of $T_{-1}^{u}(x)$ and $T_{1}^{v}(x)$ for a general solution. We will show that for large enough $L$ these functions are analytic inside the physical strip $-1 / 2+\epsilon<$ $\operatorname{Im} x<1 / 2-\epsilon$ where $\epsilon \rightarrow 0$ when $L \rightarrow \infty$. We start from eq.(158) and rewrite it as

$$
T_{-1}^{u}=+e^{-L \cosh (\pi x)} \frac{\left(T_{0}^{u-} \mathcal{Q}_{u}-T_{-1}^{u} \mathcal{Q}_{u}^{++}\right) \bar{\Phi}_{u} T_{-1}^{v}}{\overline{\mathcal{Q}}_{u}\left(\Phi_{u}^{+} \Phi_{v}^{-} \bar{\Phi}_{v}^{+} \bar{\Phi}_{u}^{-}\right)^{* s}} .
$$


and similar for $T_{+1}^{v}$. Solving for $T_{-1}^{u}$ we get

$$
T_{-1}^{u}=\frac{e^{-L \cosh (\pi x)} T_{0}^{u-} \mathcal{Q}_{u}}{\bar{Q}_{u}\left(\Phi_{u}^{+} \Phi_{v}^{-} \bar{\Phi}_{v}^{+} \bar{\Phi}_{u}^{-}\right)^{* s}-e^{-L \cosh (\pi x)} \bar{\Phi}_{u} T_{-1}^{v} \mathcal{Q}_{u}^{++}}
$$

Since $\bar{\Phi}_{u}$ is regular in the lower half plane and $T_{-1}^{v}$ is regular in the strip $-1<\operatorname{Im} x<1$ where $\left(\Phi_{u}^{+} \Phi_{v}^{-} \bar{\Phi}_{v}^{+} \bar{\Phi}_{u}^{-}\right)^{* s}$ is also regular the singularities of $T_{-1}^{u}$ for $-1<\operatorname{Im} x<0$ could be only due to zeros of the denominator.

As far as $L$ or $x$ are large for $-1 / 2<\operatorname{Im} x<0$, the second term in the denominator is exponentially suppressed and to get a zero of the denominator we should be close to a zero of $\bar{Q}_{u}$. The points close to $\bar{u}_{j}$ where the denominator vanishes are in fact $x=u_{j}$ as follows from the auxiliary finite volume BAE eq.(70). However these poles cancel with zeroes of the numerator rendering $T_{-1}$ regular at these points, a result we were familiar with already. Thus, we see that for large $L$ the only poles that could appear must lie close to the border of the physical strip $\operatorname{Im} x=-1 / 2$ where the exponent is oscillating. It oscillates faster for large $x$ and we thus have poles condensing at infinity along the borders of the physical strip.

We conclude that for the general solution - at least for large $L$ 's - the function $T_{-1}^{u}(x)$ is analytic inside almost the whole physical strip and could have poles only very close to the border. For small $L$ it can probably happen that the singularities approach the real axe. That could indicate some singular behavior of the energy levels as functions of $L$ such as the one observed in [56] (see e.g. figure 10 in this work). It would be interesting to investigate these points in greater detail.

\section{E Details on Lüscher formulae derivation}

If this section we shall present some details of the computation of the first finite volume correction to the asymptotic auxiliary Bethe equations, obtained by expanding (70) to the leading order (see section 3.2 for notation). We start by writing

$$
P\left(U_{j}\right)=\exp \left[K_{1}\left(U_{j}-i / 2+i 0\right) * \rho_{u}-K_{1}\left(U_{j}+i / 2+i 0\right) * \bar{\rho}_{u}\right] .
$$

Notice that we introduced the $i 0$ 's because $P(x)$ was originally defined in (71) for $x$ in the upperhalf-plane. Removing the $i 0$ 's by the use the Sokhatsky-Weierstrass formula we get

$$
P\left(U_{j}\right)=\exp \left[K_{1}^{-} * \rho_{u}-K_{1}^{+} * \bar{\rho}_{u}+Y_{0} / 2\right]_{x=U_{j}},
$$

where the convolutions are understood in the principal part sense and $\rho_{u}+\bar{\rho}_{u}=\log \left(1+Y_{0}\right) \simeq Y_{0}$

was used. Next we split the density $\rho_{u}$ into $\rho_{u}^{(1)}$ and $\rho_{u}^{(2)}$ as explained in section (3.2). The former contribution is purely imaginary and therefore it contributes to the exponent as

$$
K_{1}^{-} * \rho_{u}^{(1)}-K_{1}^{+} *\left(-\rho_{u}^{(1)}\right)=K_{2} * \rho_{u}^{(1)} .
$$

Hence we finally obtain

$$
P\left(U_{j}\right)=\exp \left(K_{2} * \rho^{(1)}+\left(K_{1}^{-} * \rho^{(2)}-\text { c.c. }\right)+\frac{Y_{0}}{2}\right)_{x=U_{j}},
$$


with all convolutions understood as principal part integrals. It turns out that the first and the last terms in this exponent simply convert the Bethe roots $u_{j}$ in (70) into their real parts, namely,

$$
\begin{aligned}
\frac{\mathcal{Q}_{u}^{++}\left(u_{i}\right)}{\overline{\mathcal{Q}}_{u}^{--}\left(u_{i}\right)} \exp \left(K_{2} * \rho^{(1)}\left(u_{i}\right)\right) & =\frac{\mathfrak{Q}_{u}^{++}\left(u_{i}\right)}{\mathfrak{Q}_{u}^{--}\left(u_{i}\right)} \\
\frac{\phi^{-}\left(u_{i}\right)}{\phi^{+}\left(u_{i}\right)} \frac{\mathfrak{Q}_{u}^{++}\left(u_{i}\right)}{\mathfrak{Q}_{u}^{--}\left(u_{i}\right)} \exp \left(\frac{Y_{0}\left(U_{i}\right)}{2}\right) & =\frac{\phi^{-}\left(U_{i}\right)}{\phi^{+}\left(U_{i}\right)} \frac{\mathfrak{Q}_{u}^{++}\left(U_{i}\right)}{\mathfrak{Q}_{u}^{--}\left(U_{i}\right)} .
\end{aligned}
$$

The check of the first equality goes exactly as in (78) and we will therefore not consider it here. Let us explain how to check the second equality. Notice that this expression is equivalent to

$$
\Delta u_{j} \partial_{x} \log \left(\frac{\phi^{-}(x)}{\phi^{+}(x)} \frac{\mathcal{Q}_{u}^{++}(x)}{\mathcal{Q}_{u}^{--}(x)}\right)_{x=U_{j}}=-\frac{Y_{0}\left(U_{j}\right)}{2} .
$$

Next, we write the right hand side containing $Y_{0} \simeq T_{-1} T_{1} / \phi^{+} \phi^{-}$as

$$
-\frac{Y_{0}\left(U_{j}\right)}{2}=\left(-\frac{\mathcal{Q}_{u}^{++}\left(U_{j}\right) T_{-1}^{u}\left(U_{j}\right)}{2 \phi^{+}\left(U_{j}\right) \mathcal{Q}_{u}^{\prime}\left(U_{j}\right)}\right)\left(\frac{T_{1}^{u}\left(U_{j}\right) \mathcal{Q}_{u}^{\prime}\left(U_{j}\right)}{\mathcal{Q}_{u}^{++}\left(U_{j}\right) \phi^{-}\left(U_{j}\right)}\right) .
$$

The first factor in the r.h.s. is precisely $\Delta u_{j}$. This can be seen from expanding the second equation from (22) at $x=\bar{u}_{j}=u_{j}-2 \Delta u_{j}$ to leading order in $\Delta u_{j}$. Alternatively we can find the imaginary part of $u_{j}$ by imposing regularity on the density (75) at $x=u_{j}$. To simplify the second factor in (187) we write

$$
T_{1}^{u}\left(U_{j}\right)=\left.\frac{\left(\mathcal{Q}_{u}^{++} \phi^{-}+\mathcal{Q}_{u}^{--} \phi^{+}\right)^{\prime}}{\mathcal{Q}_{u}^{\prime}}\right|_{x=U_{j}}
$$

Evaluating the derivative of the numerator and using the leading order auxiliary Bethe equations $\mathcal{Q}_{u}^{--}\left(U_{j}\right) \phi^{+}\left(U_{j}\right)+\mathcal{Q}_{u}^{++}\left(U_{j}\right) \phi^{-}\left(U_{j}\right) \simeq 0$ we find

$$
\frac{T_{1}^{u}\left(U_{j}\right) \mathcal{Q}_{u}^{\prime}\left(U_{j}\right)}{\mathcal{Q}_{u}^{++}\left(U_{j}\right) \phi^{-}\left(U_{j}\right)}=\partial_{x} \log \left(\frac{\phi^{-}(x)}{\phi^{+}(x)} \frac{\mathcal{Q}_{u}^{++}(x)}{\mathcal{Q}_{u}^{--}(x)}\right)_{x=U_{j}}
$$

thus identifying the second factor in the left hand side of (186) and completing our proof. Therefore the expansion of the auxiliary Bethe equation (70) simply reduces to (80), as announced in the main text.

To simplify the Bethe equations (79) and (80) further we shall relate the convolutions in these expressions to particular derivatives of the $Y$-function $Y_{0}$. To compute these derivatives it is useful to notice that we can write $Y_{0}$ in terms of two simple pure phase functions $a_{u}$ and $a_{v}$,

$$
a_{w}(x)=S(x) \frac{\mathcal{Q}_{w}^{+}(x)}{\mathcal{Q}_{w}^{-}(x)} e^{i L / 2 \sinh (\pi x)}
$$

as

$$
Y_{0}=\left(a_{u}^{+}+1 / a_{u}^{-}\right)\left(a_{v}^{+}+1 / a_{v}^{-}\right) .
$$

In this form, it is easy to compute the derivative of $Y_{0}$ with respect to $\theta_{k}, u_{k}$ or $v_{k}$ because we can use a simple identity

$$
\partial_{\theta_{i}} a_{w}(x)=-\pi i K_{0}\left(\theta_{i}-x\right) a_{w}(x), \quad \partial_{w_{i}} a_{w}(x)=2 \pi i K_{1}\left(w_{i}-x\right) a_{w}(x) .
$$

Furthermore if we notice that the densities $\rho^{(2)}$ can also be simply expressed in terms of these new functions as

$$
\rho_{u}^{(2)}=a_{u}^{+}\left(a_{v}^{+}+1 / a_{v}^{-}\right), \rho_{v}^{(2)}=a_{v}^{+}\left(a_{u}^{+}+1 / a_{u}^{-}\right),
$$

It is then a straightforward exercise to check the identities (83). 


\section{References}

[1] T. Matsubara, "A New Approach To Quantum Statistical Mechanics," Prog. Theor. Phys. 14 (1955) 351.

[2] A. Abrikosov, L. Gor'kov, I. Dzyaloshinskii, "Quantum field theoretical methods in statistical physics" (2 ed., Pergamon, 1965).

[3] M. Lüscher, "Volume Dependence of the Energy Spectrum in Massive Quantum Field Theories. 2. Scattering States," Commun. Math. Phys. 105 (1986) 153.

[4] M. Lüscher, "Volume Dependence Of The Energy Spectrum In Massive Quantum Field Theories. 1. Stable Particle States," Commun. Math. Phys. 104 (1986) 177.

[5] Z. Bajnok and R. A. Janik, "Four-loop perturbative Konishi from strings and finite size effects for multiparticle states," Nucl. Phys. B 807 (2009) 625 [arXiv:0807.0399 [hep-th]]. • Z. Bajnok, R. A. Janik and T. Lukowski, "Four loop twist two, BFKL, wrapping and strings," arXiv:0811.4448 [hep-th].

[6] C. Destri and H. J. de Vega, "Light cone lattices and the exact solution of chiral fermion and sigma models", J. Phys. A 22 (1989) 1329. • C. Destri and H. J. de Vega, "Light cone lattice approach to fermionic theories in 2D: the massive Thirring model," Nucl. Phys. B 290 (1987) 363.

[7] D. Fioravanti, A. Mariottini, E. Quattrini and F. Ravanini, "Excited state Destri-De Vega equation for sine-Gordon and restricted sine-Gordon models," Phys. Lett. B 390 (1997) 243 [arXiv:hep-th/9608091].

[8] A. Hegedus, F. Ravanini and J. Suzuki, "Exact finite size spectrum in super sine-Gordon model," Nucl. Phys. B 763, 330 (2007) [arXiv:hep-th/0610012].

[9] G. Feverati, "Finite volume spectrum of sine-Gordon model and its restrictions," arXiv:hepth/0001172.

[10] P. A. Pearce, L. Chim and C. r. Ahn, "Excited TBA equations. I: Massive tricritical Ising model," Nucl. Phys. B 601 (2001) 539 [arXiv:hep-th/0012223].

[11] A. Hegedus, F. Ravanini and J. Suzuki, "Exact finite size spectrum in super sine-Gordon model," Nucl. Phys. B 763 (2007) 330 [arXiv:hep-th/0610012].

$[12]$ G. Feverati, F. Ravanini and G. Takacs, "Nonlinear integral equation and finite volume spectrum of minimal models perturbed by Phi(1,3)," Nucl. Phys. B $5 \mathbf{7 0}$ (2000) 615 [arXiv:hepth/9909031]. - G. Feverati, F. Ravanini and G. Takacs, "Scaling functions in the odd charge sector of sine-Gordon/massive Thirring theory," Phys. Lett. B 444 (1998) 442 [arXiv:hepth/9807160]. - G. Feverati, F. Ravanini and G. Takacs, "Nonlinear integral equation and finite volume spectrum of sine-Gordon theory," Nucl. Phys. B 540 (1999) 543 [arXiv:hepth/9805117]. • G. Feverati, F. Ravanini and G. Takacs, "Truncated conformal space at c $=1$, nonlinear integral equation and quantization rules for multi-soliton states," Phys. Lett. B 430 (1998) 264 [arXiv:hep-th/9803104].

[13] J. Balog and A. Hegedus, "TBA equations for excited states in the sine-Gordon model," J. Phys. A 37 (2004) 1903 [arXiv:hep-th/0304260]. 
[14] Y. k. Zhou and P. A. Pearce, "Solution of functional equations of restricted A(n-1)(1) fused lattice models," Nucl. Phys. B 446, 485 (1995) [arXiv:hep-th/9502067].

[15] P. Zinn-Justin, "Non-linear integral equations for complex affine Toda models associated to simply laced Lie algebras," J. Phys. A 31, 6747 (1998) [arXiv:hep-th/9712222].

[16] A.Klümper and P.A.Pearce, Analytic calculation of scaling dimensions: tricritical hard squares and critical hard hexagons, J. Stat. Phys. 64, 13-76 ( 1991) • A.Klumper, M.T.Batchelor and P.A.Pearce, Central charges of the 6- and 19-vertex models with twisted boundary conditions, J. Phys. A 24, 3111-3133 (1991).

[17] Al. B. Zamolodchikov, "Thermodynamic Bethe ansatz in relativistic models: Scaling 3-state potts and Lee-Yang models" Nuclear Physics B, Volume 342, Issue 3, 8 October 1990, Pages 695-720

[18] Al. B. Zamolodchikov, "TBA equations for integrable perturbed SU(2)-k x SU(2)-1 / SU(2)-k+ l coset models," Nucl. Phys. B 366 (1991) 122.

[19] A. Kuniba, T. Nakanishi and J. Suzuki, "Functional relations in solvable lattice models. 1: Functional relations and representation theory," Int. J. Mod. Phys. A 9 (1994) 5215 [arXiv:hepth/9309137].

[20] Al. B. Zamolodchikov, "On the thermodynamic Bethe ansatz equations for reflectionless ADE scattering theories," Phys. Lett. B 253 (1991) 391. • Al. B. Zamolodchikov, "From tricritical Ising to critical Ising by thermodynamic Bethe ansatz," Nucl. Phys. B 358 (1991) 524. - Al. B. Zamolodchikov, "Thermodynamic Bethe ansatz for RSOS scattering theories," Nucl. Phys. B 358 (1991) 497. • Al. B. Zamolodchikov, "Thermodynamics of imaginary coupled sine-Gordon: Dense polymer finite size scaling function," Phys. Lett. B 335 (1994) 436. - Al. B. Zamolodchikov, "Painleve III and 2-d polymers," Nucl. Phys. B 432 (1994) 427 [arXiv:hep-th/9409108].

[21] M. J. Martins, "The Thermodynamic Bethe Ansatz For Deformed W A(N)-1 Conformal Field Theories," Phys. Lett. B 277 (1992) 301 [arXiv:hep-th/9201032].

[22] P. Fendley, "Excited state thermodynamics," Nucl. Phys. B 374, 667 (1992) [arXiv:hepth/9109021].

[23] V. A. Fateev and A. B. Zamolodchikov, "Integrable perturbations of Z(N) parafermion models and O(3) sigma model," Phys. Lett. B 271 (1991) 91.

[24] V. V. Bazhanov, S. L. Lukyanov and A. B. Zamolodchikov, "Integrable structure of conformal field theory, quantum KdV theory and thermodynamic Bethe ansatz," Commun. Math. Phys. 177 (1996) 381 [arXiv:hep-th/9412229].

[25] P. Dorey and R. Tateo, "Excited states by analytic continuation of TBA equations," Nucl. Phys. B 482 (1996) 639 [arXiv:hep-th/9607167]. • P. Dorey and R. Tateo, "Anharmonic oscillators, the thermodynamic Bethe ansatz, and nonlinear integral equations," J. Phys. A 32 (1999) L419 [arXiv:hep-th/9812211].

[26] J. Balog and A. Hegedus, "TBA equations for excited states in the $\mathrm{O}(3)$ and $\mathrm{O}(4)$ nonlinear sigma-model," J. Phys. A 37 (2004) 1881 [arXiv:hep-th/0309009]. 
[27] J. Balog and A. Hegedus, "TBA equations for the mass gap in the $\mathrm{O}(2 \mathrm{r})$ non-linear sigmamodels," Nucl. Phys. B 725 (2005) 531 [arXiv:hep-th/0504186].

[28] J. Balog and A. Hegedus, "Virial expansion and TBA in $\mathrm{O}(\mathrm{N})$ sigma models," Phys. Lett. B 523 (2001) 211 [arXiv:hep-th/0108071].

[29] A. Hegedus, "Nonlinear integral equations for the finite size effects of RSOS and vertex-models and related quantum field theories," Nucl. Phys. B 732 (2005) 463 [arXiv:hep-th/0507132].

[30] A. Hegedus, "Nonlinear integral equations for finite volume excited state energies of the $\mathrm{O}(3)$ and O(4) nonlinear sigma-models," J. Phys. A 38 (2005) 5345 [arXiv:hep-th/0412125].

[31] Al. B. Zamolodchikov, "On the thermodynamic Bethe ansatz equation in sinh-Gordon model," J. Phys. A 39, 12863 (2006) [arXiv:hep-th/0005181].

[32] J. Teschner, "On the spectrum of the Sinh-Gordon model in finite volume," Nucl. Phys. B 799 (2008) 403 [arXiv:hep-th/0702214].

[33] A. B. Zamolodchikov and A. B. Zamolodchikov, "Factorized S-matrices in two dimensions as the exact solutions of certain relativistic quantum field models," Annals Phys. 120 (1979) 253.

[34] R. Hirota, Discrete analogue of a generalized Toda equation, Journ. of the Phys. Soc. of Japan, 50 (1981) 3785-3791.

[35] I. Krichever, O. Lipan, P. Wiegmann and A. Zabrodin, "Quantum integrable models and discrete classical Hirota equations," Commun. Math. Phys. 188 (1997) 267 [arXiv:hepth/9604080]. - A. Zabrodin, Discrete Hirota's equation in quantum integrable models, Int. J. Mod. Phys. B 11 (1997) 3125-3158, arXiv.org: hep-th/9610039; • A. Zabrodin, Hirota equation and Bethe ansatz, Teor. Mat. Fys. 116 (1998) 54-100 (English translation: Theor. Math. Phys. 116 (1998) 782-819).

[36] V. V. Bazhanov, S. L. Lukyanov and A. B. Zamolodchikov, "Integrable structure of conformal field theory. III: The Yang-Baxter relation," Commun. Math. Phys. 200 (1999) 297 [arXiv:hepth/9805008].

[37] N. Beisert, B. Eden and M. Staudacher, "Transcendentality and crossing," J. Stat. Mech. 0701 (2007) P021 [arXiv:hep-th/0610251].

[38] R. A. Janik, "The AdS(5) x $\mathrm{S}^{* *} 5$ superstring worldsheet S-matrix and crossing symmetry," Phys. Rev. D 73, 086006 (2006) [arXiv:hep-th/0603038].

[39] V. Kazakov, A. Sorin and A. Zabrodin, "Supersymmetric Bethe ansatz and Baxter equations from discrete Hirota dynamics," Nucl. Phys. B 790, 345 (2008) [arXiv:hep-th/0703147]; • A. Zabrodin, "Backlund transformations for difference Hirota equation and supersymmetric Bethe ansatz," arXiv:0705.4006 [hep-th]. • V. Kazakov and P. Vieira, "From Characters to Quantum (Super)Spin Chains via Fusion," JHEP 10 (2008) 050 [JHEP 0810 (2008) 050] [arXiv:0711.2470 [hep-th]];

[40] L. D. Faddeev and N. Y. Reshetikhin, "Integrability Of The Principal Chiral Field Model In (1+1)-Dimension," Annals Phys. 167 (1986) 227.

[41] A. M. Polyakov and P. B. Wiegmann, "Theory of nonabelian Goldstone bosons in two dimensions," Phys. Lett. B 131 (1983) 121. 
[42] P. Wiegmann, "On the theory of nonabelian goldstone bosons in two dimensions; exact solution of the $S U(N) \times S U(N)$ nonlinear $\sigma$ model", Phys. Lett. B 141 (1984) 217.

[43] P. Wiegmann, "Exact Factorized S Matrix Of The Chiral Field In Two-Dimensions," Phys. Lett. B 142 (1984) 173.

[44] V. V. Bazhanov, S. L. Lukyanov and A. B. Zamolodchikov, "Quantum field theories in finite volume: Excited state energies," Nucl. Phys. B 489 (1997) 487 [arXiv:hep-th/9607099].

[45] V. A. Fateev, "The sigma model (dual) representation for a two-parameter family of integrable quantum field theories," Nucl. Phys. B 473 (1996) 509.

[46] D. S. Shin, "A determination of the mass gap in the $O(n)$ sigma-model," Nucl. Phys. B 496 (1997) 408 [arXiv:hep-lat/9611006]. • D. S. Shin, "Correction to four-loop RG functions in the two-dimensional lattice O(n) sigma-model," Nucl. Phys. B 546 (1999) 669 [arXiv:heplat/9810025].

[47] R. A. Janik and T. Lukowski, "Wrapping interactions at strong coupling - the giant magnon," Phys. Rev. D 76 (2007) 126008 [arXiv:0708.2208 [hep-th]].

[48] G. Arutyunov and S. Frolov, "On String S-matrix, Bound States and TBA," JHEP 0712, 024 (2007) [arXiv:0710.1568 [hep-th]].

[49] J. Ambjorn, R. A. Janik and C. Kristjansen, "Wrapping interactions and a new source of corrections to the spin-chain string duality," Nucl. Phys. B 736, 288 (2006) [arXiv:hep-th/0510171].

[50] V. A. Fateev, V. A. Kazakov and P. B. Wiegmann, "Principal Chiral Field At Large N," Nucl. Phys. B 424 (1994) 505 [arXiv:hep-th/9403099].

[51] N. Mann and J. Polchinski, "Bethe Ansatz for a Quantum Supercoset Sigma Model," Phys. Rev. D 72 (2005) 086002 [arXiv:hep-th/0508232].

[52] Z. Tsuboi, Analytic Bethe ansatz and functional equations for Lie superalgebra sl $(r+1 \mid s+1)$, J. Phys. A: Math. Gen. 30 (1997) 7975-7991. • Z. Tsuboi, Analytic Bethe ansatz related to a oneparameter family of finite-dimensional representations of the Lie superalgebra $s l(r+1 \mid s+1)$, J. Phys. A: Math. Gen. 31 (1998) 5485-5498. • Z. Tsuboi, Analytic Bethe ansatz and functional equations associated with any simple root systems of the Lie superalgebra sl $(r+1 \mid s+1)$, Physica A 252 (1998) 565-585. •Z. Tsuboi, Nonlinear integral equations and high temperature expansion for the $U_{q}(\widehat{s l}(r+1 \mid s+1))$ Perk-Schultz model, Nucl. Phys. B737 (2006) 261-290, arXiv.org: cond-mat/0510458.

[53] G. Juttner, A. Klumper and J. Suzuki, "From fusion hierarchy to excited state TBA," Nucl. Phys. B 512 (1998) 581 [arXiv:hep-th/9707074].

[54] V. V. Bazhanov and Z. Tsuboi, "Baxter's Q-operators for supersymmetric spin chains," Nucl. Phys. B 805 (2008) 451 [arXiv:0805.4274 [hep-th]].

[55] N. Gromov, V. Kazakov, K. Sakai and P. Vieira, "Strings as multi-particle states of quantum sigma-models," Nucl. Phys. B 764 (2007) 15 [arXiv:hep-th/0603043].

[56] P. Dorey, M. Pillin, A. Pocklington, I. Runkel, R. Tateo and G. M. T. Watts, "Finite size effects in perturbed boundary conformal field theories," arXiv:hep-th/0010278. 

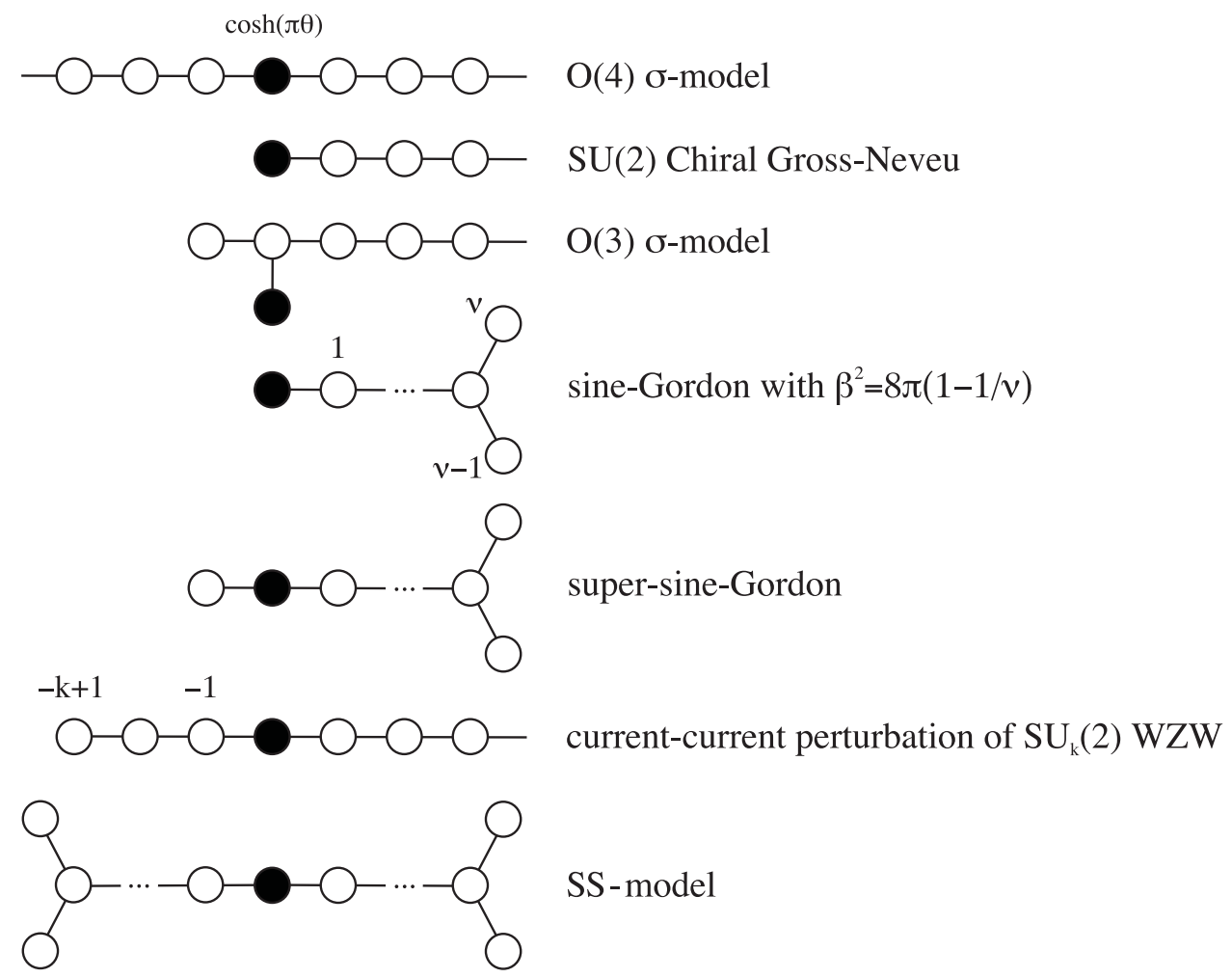

SS-model

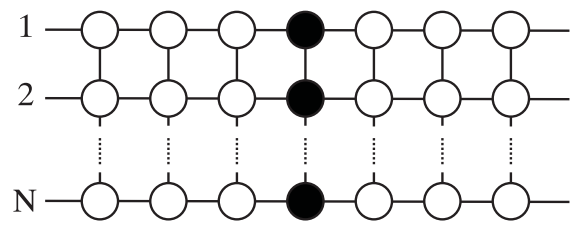

SU(N+1) Principal ChiraL Field

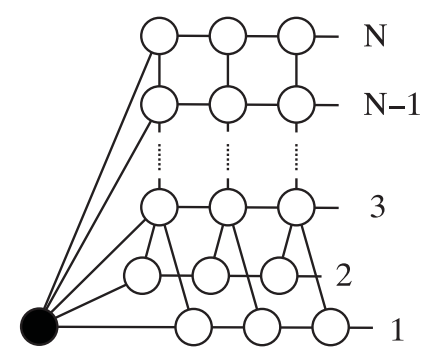

$\mathrm{SO}(2 \mathrm{~N}) \sigma$-model

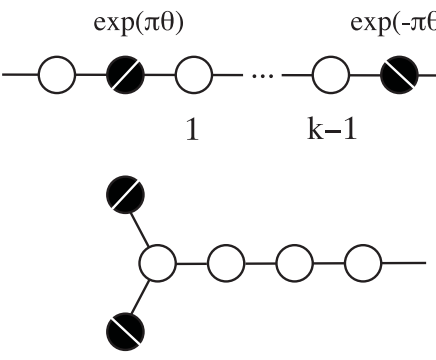

WZW SU $(2)$ off-critical

Figure 8: For several models the Y-system TBA equations for the ground state energy are known and can be represented by the diagrams such as the ones in this figure. Using the techniques developed in this paper it would be extremely interesting to compute their complete spectrum in a systematic way. 